\title{
TASI Lectures on WIMPs and Supersymmetry
}

\author{
Patrick J. Fox* \\ Theoretical Physics Department, Fermilab, Batavia, IL 60510, USA \\ E-mail: pjfoxefnal.gov
}

\begin{abstract}
Weakly interacting massive particles (WIMPs) are an appealing and well studied candidate for dark matter (DM) that arise in many extensions of the standard model. I will discuss where a WIMP sits in the catalogue of DM candidates, its origin as a thermal relic, and the various approaches available to discover it. The prototypical example of a WIMP is the neutralino of the minimal supersymmetric standard model (MSSM). I will give an introduction to the formalism and phenomenology of supersymmetry and the MSSM, with emphasis on DM. These notes are the combination of lectures given at TASI 2018 and a Joint ICTP-Trieste/ICTP-SAIFR School.
\end{abstract}

Theoretical Advanced Study Institute Summer School 2018 'Theory in an Era of Data'(TASI2018) 4 - 29 June, 2018

Boulder, Colorado

${ }^{*}$ Speaker. 


\section{Contents}

1. Introduction 2

1.1 Evidence and properties of Dark Matter 2

1.2 Possibilities for Dark Matter 5

2. WIMPs 6

2.1 Freeze out 6

$\begin{array}{lll}2.2 \text { Hot relic } & 10\end{array}$

$\begin{array}{lll}2.3 \text { Cold relic } & 10\end{array}$

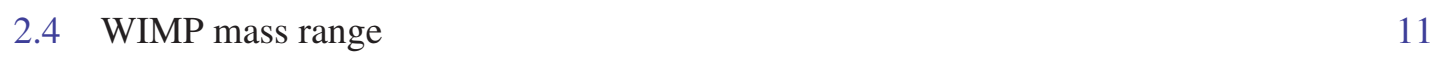

2.5 Summary of WIMPs 11

3. Supersymmetry 12

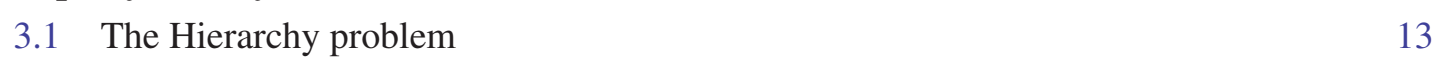

$\begin{array}{ll}3.2 & \text { Gauge coupling unification } \\ 3.3 & 14\end{array}$

$\begin{array}{lll}3.3 & \text { Superfield (and other) formalism } & 15\end{array}$

$\begin{array}{lll}3.4 & \text { Superspace } & 16\end{array}$

$\begin{array}{llr}3.5 & \text { Chiral superfield } & 18\end{array}$

$\begin{array}{lll}3.6 & \text { Vector superfield } & 20\end{array}$

$\begin{array}{lll}3.7 & \text { R-symmetry } 23\end{array}$

3.8 Putting the formalism to work: O'Raifeartaigh and other models 23

4. The MSSM 25

4.1 SUSY breaking in the MSSM 29

$\begin{array}{lll}4.2 \text { Spurions } & 29\end{array}$

$\begin{array}{lll}4.3 & \text { Superpartner mass spectra } & 31\end{array}$

5. Supersymmetric Dark Matter

5.1 Freeze out revisited 36

$\begin{array}{lll}5.2 & \text { Direct detection } & 38\end{array}$

5.3 Indirect detection 44

5.4 Capture in the Sun 46

5.5 WIMPs at colliders 49

6. Conclusions $\quad 50$ 


\section{Introduction}

There is now overwhelming evidence that the majority of the matter in the universe is from particles not present in the standard model (SM). One of the most appealing candidates for this dark matter (DM) is a weakly interacting massive particle (WIMP). WIMPs have the virtue that their interactions with the SM allow them to be made through thermal processes in the early universe. A weak scale interaction with the SM leads to a relic amount of DM that is consistent with present day observations. Furthermore, WIMP DM candidates arise in many extensions of the SM whose motivations are often independent of those of DM. The quintessential example of this is supersymmetry and the minimal supersymmetric extension of the SM (MSSM). Extending the SM to contain superpartners of all the SM fields is often motivated by trying to solve the hierarchy problem. The doubling of the spectrum contains within it a DM candidate with all the necessary features to be a thermal relic candidate and a WIMP. These notes, which are the amalgamation of lectures given at TASI 2018 and a Joint ICTP-Trieste/ICTP-SAIFR School, will attempt to introduce the concepts of WIMPs, supersymmetry, and the phenomenology of WIMP DM within the MSSM.

They start by reviewing the evidence for DM and describing the range of possibilities for what it can be. In Section 2 we will focus on thermal relics, solve the Boltzmann equation and narrow this range down to the WIMP candidate. In Section 3 we will take a detour to discuss supersymmetry, (some of) its motivations, and the superspace and superfield formalism. In Section 4 we put this formalism to work to describe the MSSM and its phenomenology, with particular focus on the electroweakino sector that gives the DM candidate. In Section 5 we return to WIMP DM and the various ways we hope to detect it - directly, indirectly, and at colliders - illustrating these techniques with examples from the MSSM. Finally, in Section 6 we motivate continued study. The notes will be sprinkled with simple exercises meant to illustrate or extend the concepts discussed in the text.

\subsection{Evidence and properties of Dark Matter}

A census of the entire universe shows that the standard model makes up about $4 \%$ of the matter/energy of the universe, with about $0.4 \%$ being locked up in stars and planets and the remaining $3.6 \%$ being in inter-galactic gas. Dark energy, the mysterious "stuff" driving the present-day expansion of the universe makes up around $73 \%$ of the budget. The final $23 \%$ is dark matter, which behaves under gravity as SM matter does i.e. it clumps, but it does not couple as strongly as SM matter under the gauge interactions. All of this information has been gleamed through DM's gravitational interactions at various length scales and at various times. We now briefly discuss some of these probes of DM.

Motions of Stars and Galaxies In the 1930's Zwicky measured the motion of galaxies in the Coma cluster. He applied the virial theorem, the fact that the average kinetic and potential energy are related by $\langle T\rangle=-\langle V\rangle / 2$, to the measured speeds to infer the gravitational potential that the galaxies appeared to move in. As an alternative way to measure this potential he calculated the mass of the galaxies from their luminosities. When comparing the two methods he found a large discrepancy and concluded that up to $90 \%$ of the cluster must be matter that does not shine: dark matter. 
In the 1970's Rubin measured the motion of the outermost stars in spiral galaxies. From their orbital speeds she determined the force holding them in orbit and thus the amount of matter interior to their orbit, $M(r)$. For circular orbits the speed is $v(r)=\sqrt{G M(r) / r}$. Based on the visible material in galaxies one would conclude that for the outermost stars, where $M(r) \rightarrow$ const., the speeds should be decreasing with radius. Instead what was observed was a flat rotation curve, i.e. $v(r) \rightarrow$ const.. Again leading one to conclude there was additional material, distributed as $M(r) \sim r$ at large $r$, in spiral galaxies.

More recently, observations have been made of the aftermath of the merging of two or more galaxy clusters, the most famous being the Bullet Cluster. Images of these systems have been made in multiple wavelengths. By measuring in X-rays one can determine the location of the hot gas in the system. The location of the mass is determined by looking at weak lensing effects. Comparing the position of the galaxies, gas, and mass places bounds on the DM self interactions with collisionless DM behaving more like the galaxies and DM with large self interactions behaving as the gas. The bullet cluster requires that the self scattering cross section obeys $\sigma_{\chi \chi} / m \lesssim 1 \mathrm{~cm}^{2} / \mathrm{g}$.

Cosmic Microwave Background The cosmic background radiation ${ }^{1}$ left over from the big bang allows us to observe the universe back to when protons and electrons recombined to form neutral hydrogen at $z \sim 1100$, approximately 380,000 years after the big bang when the temperature was $T \sim \mathrm{eV}$. The spectrum of this radiation is a very precise black body with a temperature that has now redshifted to $T=2.73 \mathrm{~K}$. It is very isotropic with temperature fluctuations of $\delta T / T \sim 10^{-5}$, with these originating from quantum fluctuations of the inflaton. These temperature fluctuations are often decomposed into spherical harmonics,

$$
\delta T(\theta, \phi)=\sum_{\ell, m} a_{\ell m} Y_{\ell m}(\theta, \phi) .
$$

A useful quantity to study is the power spectrum of the temperature fluctuations $C_{\ell}=$ $\frac{1}{2 \ell+1} \sum_{m}\left|a_{\ell m}\right|^{2}$. This has been measured across the full sky as well as to very high precision at small angular scales (high $\ell$ ) over sections of the sky, see Figure 1 . The wiggles in the power spectrum can be well fit by a $\Lambda$ CDM model with six free parameters, one of which is the DM density. The origin of the wiggles is the growth of structure after matter-radiation equality at $z \sim 2700$. If the only seeds for structure formation were baryonic overdensities then the growth could not occur until photon-baryon decoupling. Instead if there is a component of matter which is not coupled to photons it can start linear growth after matter-radiation equality. The wiggles are well fit for a cosmology which contains DM, see Figure 1.

In addition to the CMB power spectrum we can measure related physics through CMB polarization as well as Large Scale Structure and Baryon Acoustic Oscillations. All these measurements are in good accord and all point towards the need for a sizable DM component.

Question 1. The Milky Way The Milky Way is approximately made up of a disk of stars, a central bulge, and a spherical Dark Matter halo. What are the approximate sizes of each of these components? Consider a galaxy made of a uniform disk of stars of constant density $\rho$,

\footnotetext{
${ }^{1}$ For a nice set of lectures on aspects of the CMB see [1].
} 

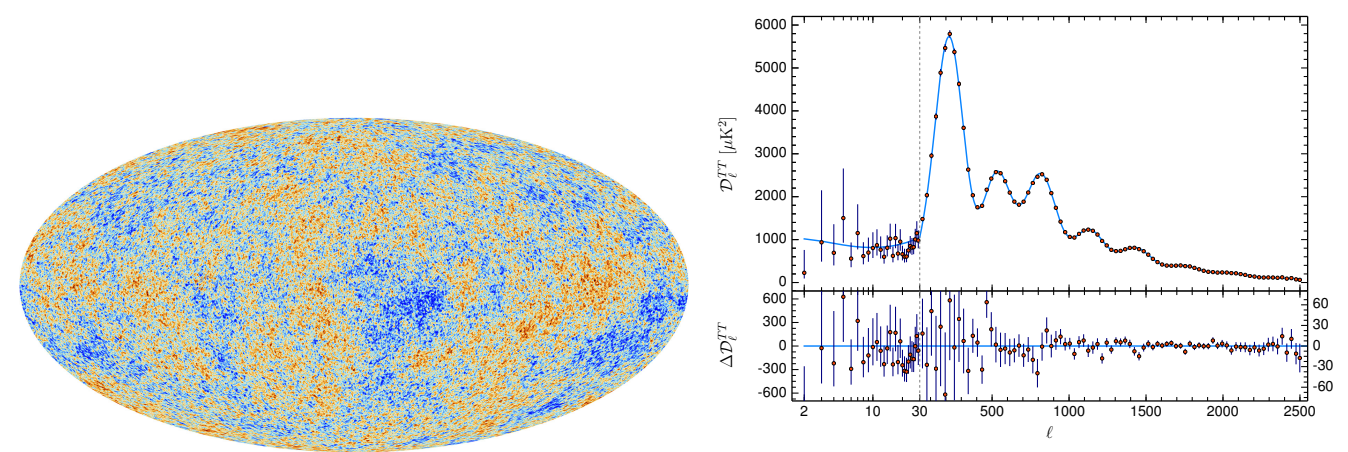

Figure 1: Left: CMB temperature anisotropies as observed by Planck. Right: the CMB power spectrum along with the best fit $\Lambda \mathrm{CDM}$ prediction (light blue); taken from [2].

and size $R$ and thickness $h$. Determine the orbital speed of objects in the plane of the disk, as a function of $r$. Compare this to the observations of Vera Rubin, and others, determine the radial distribution of the Dark Matter, assuming it is spherically distributed and constant density.

Big Bang Nuclear Synthesis The universe right after the big bang is a hot soup of particles in thermodynamic equilibrium ${ }^{2}$. A few seconds after the big bang the soup is made up of protons, neutrons, electrons, photons, and neutrinos. The ratio of neutron to proton number density is determined purely by thermodynamics and $n / p \sim e^{-\Delta m / T}$, with the mass splitting $\Delta m \sim 1.3 \mathrm{MeV}$. Around $\sim 1 \mathrm{~s}$ after the big bang the universe has cooled to $T \sim 0.8 \mathrm{MeV}$. The weak interactions (e.g. $v+n \rightleftharpoons p+e$ ) are no longer faster than the expansion rate of the universe and the neutrons and protons drop out of chemical equilibrium. The jargon we use is the weak interactions "freeze out", with $n / p \sim 1 / 6$. At this time the baryon to photon ratio is tiny, $\eta=n_{b} / n_{\gamma} \sim 6 \times 10^{-10}$.

As the temperature drops further one would expect the neutrons and protons to combine to form heavier elements, but the path for this is blocked. In order to go from free neutrons and protons to heavier elements, such as helium, lithium, etc, one must go through deuterium via $p+n \rightarrow \mathrm{D}+\gamma$. However, although the binding of deuterium $\Delta_{D}=2.2 \mathrm{MeV}$ is below the energy of the average photon there are so many more photons than baryons that there are a sufficient number of photons above the binding energy to dissociate deuterium as soon as it is formed. Thus, there is a "deuterium bottleneck" which means no deuterium can form until the temperature is low enough that there is less than one photon of energy $\Delta_{D}$ per baryon,

$$
\eta^{-1} e^{-\Delta_{D} / T} \lesssim 1
$$

It takes about 100s for the universe to cool sufficiently, to $T \sim 0.1 \mathrm{MeV}$, in which time some of the neutrons have decayed, changing the neutron to proton ratio to $n / p \sim 1 / 7$. Once clear of the bottle neck heavier elements form. To a very good approximation all neutrons become

\footnotetext{
${ }^{2}$ It was a pleasure to discuss this in Boulder given the first detailed analysis was carried out locally [3].
} 
bound up in the first element beyond hydrogen, helium, with two neutrons per atom. Thus, we can make a prediction for the primordial mass fraction of ${ }^{4} \mathrm{He}, Y_{p}$,

$$
Y_{p} \equiv \frac{\rho_{4} \mathrm{He}}{\rho_{b}}=\frac{4(n / 2)}{n+p}=\frac{2 n / p}{1+n / p} \approx \frac{1}{4} .
$$

This simple calculation gives a remarkable accurate answer. To do this more carefully, and to determine the primordial abundances of ${ }^{3} \mathrm{He},{ }^{7} \mathrm{Li}$, and $\mathrm{D}$, requires running complicated computer codes. However, the bottom line is that the observed primordial abundance of light elements is well explained ${ }^{3}$ by SM physics and is consistent with $\eta \sim 6 \times 10^{-10}$ implying $\Omega_{b} h^{2} \sim 0.022$. Given that the CMB indicates $\Omega_{m} h^{2} \sim 0.14$ this means that most of the matter is non-baryonic. For those wishing for a more detailed analysis of the physics of BBN, see e.g.[4].

Combining all of the evidence described above, along with others not described, we reach a consistent picture that $23 \%$ of the energy in the universe is due to dark matter and that DM is nonbaryonic, is neutral under the SM, does not interact much with the SM or itself, was non-relativistic by the time of the CMB, and it is cosmologically long lived. There is no particle in the SM with these properties so the observation of DM is the discovery of beyond the standard model (BSM) physics.

\subsection{Possibilities for Dark Matter}

There are many possibilities for what new physics makes up this DM abundance. DM could be a single new particle, a multitude of particles, or an entire new sector with DM particles, dark interactions, and as much interesting dynamics in that sector as occurs in ours. If a single particle makes up all of DM it could be a fermion (Dirac or Majorana) or a boson (scalar or vector). The mass range for fermionic DM is $m_{\text {fermionic-DM }} \gtrsim 1 \mathrm{keV}$ whereas bosonic DM can be as light as $m_{\text {bosonic-DM }} \gtrsim 10^{-22} \mathrm{eV}$. Fermionic DM cannot be lighter because Fermi degeneracy pressure would forbid DM being confined into Galaxy sized structures. Below the lower bound for bosonic dark matter the DM's de Broglie wavelength would be greater than the size of a dwarf galaxy. Rather than an entirely new particle DM could instead be a massive compact halo object (MA$\mathrm{CHO}$ ), for example a $30 M_{\odot}$ black hole [5]. The mass range of all these options is huge, it covers approximately 99 orders of magnitude!

Within this giant mass range there are many particle physics models, see Figure 2. For instance, hidden sector models, which contain both a DM particle and new dark interactions, populate the mass range $\mathrm{keV}-\mathrm{PeV}$; these will be discussed in more detail in this volume by Tongyan Lin. SIMPs [6] and Elders [7] exist for masses $\mathrm{MeV}-\mathrm{GeV}$. At lighter masses $\left(\sim 10^{-12} \mathrm{eV}-10^{-2} \mathrm{eV}\right)$ an interesting DM candidate is the QCD axion, which will be discussed in greater detail elsewhere in this volume by Anson Hook [8]. At the top end of the mass range 30 solar mass black holes are an intriguing possibility given the recent LIGO observations. However, for these lectures we will focus on the candidate that has historically been the most studied: the Weakly Interacting Massive Particle (WIMP). This, typically, is defined to live in the mass range $\sim \mathrm{GeV}-\mathscr{O}(10) \mathrm{TeV}$, we will see why below.

\footnotetext{
${ }^{3}$ The amount of lithium observed is less than expected and may be an indication of new physics, or poorly understood astrophysics.
} 

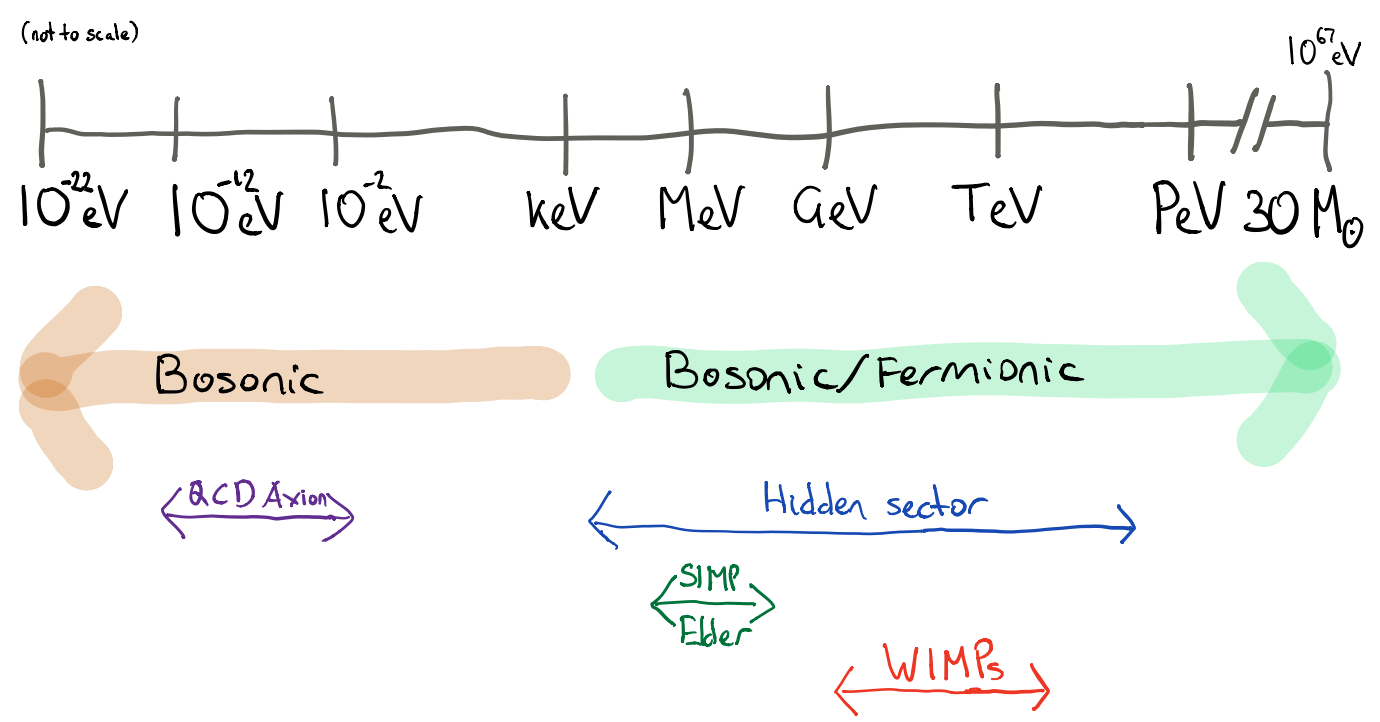

Figure 2: A cartoon of the mass range, and location of some of the candidates, for dark matter.

\section{WIMPs}

Having discussed the pantheon of DM candidates we now focus for the remainder of these lectures on the WIMP candidate. We will first spend sometime delving into the details of the Boltzmann equation, which determines the amount of DM left over once the thermal bath cools below the DM mass. The thermal relic story, combined with arguments about the size of scattering cross sections, will determine the WIMP mass range. As one would expect for such a long-studied DM particle there are many good textbooks and reviews related to dark matter. Two standard textbooks that discuss cosmology, the observations that support the DM hypothesis, and describe freeze-out, Boltzmann equations etc are the "The Early Universe" by Kolb and Turner [9] and the more up to date "Modern Cosmology" by Dodelson [10]. I also find the PDG [11] a good resource. There are also several extensive online resources, two of note are by Yann Mambrini [12] and Flip Tanedo [13]. There are previous TASI lectures on this topic e.g. $[14,15]$ and some reviews e.g. [16].

\subsection{Freeze out}

If DM has more than gravitational interactions with SM particles there may be reactions whose rate is fast enough to bring DM into thermal equilibrium in the early universe e.g. $\chi \chi \rightleftharpoons f \bar{f}$. We wish to understand how much DM is made through interactions with the SM, and how much there is today as a result. The quantity of interest is the DM phase-space density $f(\vec{p}, t)$. Since, we will assume that the early universe is well described by a Freedman-Robertson-Walker metric ${ }^{4}$, which is homogenous and isotropic, $f$ is actually only a function of $|\vec{p}|$ and $t$. The phase-space density

\footnotetext{
${ }^{4}$ In particular, $d s^{2}=d t^{2}-a^{2}(t)\left(d r^{2}+r^{2} d \Omega^{2}\right)$ and the Hubble constant is defined as $H \equiv \dot{a} / a$. See Jim Cline's lectures [17] in this volume for more details on early universe cosmology.
} 
evolution is determined by the Liouville operator

$$
\mathbf{L}[f]=\frac{\partial f}{\partial t}-\frac{\dot{a}}{a} p \frac{\partial f}{\partial p}
$$

and with interactions $f$ satisfies the Boltzmann equation $\mathbf{L}[f]=\mathbf{C}[f]$, where $\mathbf{C}[f]$ is the so called "collision term" which encodes all the interactions. In the case where the species described by $f\left(p_{1}\right)$ undergoes a two-to-two interaction, $p_{1}+p_{2} \rightleftharpoons p_{3}+p_{4}$, this collision term is given by

$$
\begin{aligned}
\mathbf{C}\left[f\left(p_{1}\right)\right]= & -\frac{1}{E_{1}} \int \prod_{i=2}^{4} d P_{i}(2 \pi)^{4} \delta^{(4)}\left(p_{1}+p_{2}-p_{3}-p_{4}\right)\left(f_{1} f_{2}\left(1 \pm f_{3}\right)\left(1 \pm f_{4}\right)|\mathscr{M}(12 \rightarrow 34)|^{2}\right. \\
& \left.-f_{3} f_{4}\left(1 \pm f_{1}\right)\left(1 \pm f_{2}\right)|\mathscr{M}(34 \rightarrow 12)|^{2}\right)
\end{aligned}
$$

with the shorthand, $d P_{i}=\frac{d^{3} p_{i}}{(2 \pi)^{3} 2 E_{i}}$. Here $\mathscr{M}$ is the matrix element for the forwards or backwards process, the \pm correspond to Bose enhancement or Fermi blocking, respectively. Before we try to solve this complicated equation in general let us try to find equilibrium solutions (i.e. $\mathbf{L}[f]=$ $\mathbf{C}[f]=0$ ) in the classical limit where we ignore the Bose/Fermi statistics. It can be shown on general grounds [18] that any such solution must have the form

$$
f_{\mathrm{eq}}=e^{\mu(t)-\beta(t) E} .
$$

In other words the equilibrium solution looks like a thermal distribution with time dependent chemical potential and temperature $(\beta \sim 1 / T)$. Since $E^{2}=p^{2}+m^{2}, \mathbf{L}[f]=0$ implies,

$$
\frac{\dot{\mu}}{\dot{\beta}}=E-\frac{\dot{a}}{a} \frac{\beta}{\dot{\beta}} \frac{p^{2}}{E} .
$$

It turns out that for the general case there is $n o \mu$ and $\beta$ that solve this equation! Which is to say, there are no equilibrium solutions to the Boltzmann equation in an expanding universe for arbitrary mass particles. This can be traced back to the lack of a spatial constant timelike Killing vector in the FRW metric, or you can just try to solve this equation. However, there are two physically interesting limits for which there are equilibrium solutions, $m \rightarrow 0$ and $m \rightarrow \infty$. If the particle is massless there is a solution to (2.4) with $\mu=0$ and $\beta \propto a$. Thus, massless particles (typically referred to as radiation) can stay in equilibrium in an expanding universe and their temperature redshifts as $T \sim 1 / a$. In the large mass limit the solution has $\mu=m \beta+$ const. and $\beta \propto a^{2}$. We see that for non-relativistic massive particles (or matter) the temperature redshifts as $T \sim 1 / a^{2}$.

So if the universe starts out at temperatures well above a particles mass, and it has sufficiently strong interactions with the bath, it will be in thermodynamic equilibrium and will maintain a thermal distribution, cooling as $1 / a$. At some point, $T \sim m$, it will no longer follow an equilibrium distribution, but if it still has strong interactions with the light degrees of freedom it will shortly thereafter equilibrate and follow a non-relativistic thermal distribution, cooling as $1 / a^{2}$.

We usually don't want to know about the full phase-space distribution of a particle and instead take moments of it. For instance the number density of a particle is given by

$$
n(t)=g \int \frac{d^{3} p}{(2 \pi)^{3}} f(p, t)
$$


where $g$ counts the internal degrees of freedom of the particle. Inserting the previously determined limiting solutions for $f_{\text {eq }}$ we see that $n_{\text {rad }} \sim T^{3}$ and $n_{\text {matter }} \sim(m T)^{3 / 2} e^{-m / T}$. Integrating both sides of the Boltzmann equation, and doing some integration by parts, leads to its more familiar form

$$
\dot{n}+3 H n=g_{1} \int \frac{d^{3} p_{1}}{(2 \pi)^{3}} \mathbf{C}\left[f\left(p_{1}\right)\right] .
$$

Even in this simpler form in general one would expect to have solve a set of coupled integrodifferential equations for each of the species involved in the reactions; an intimidating proposition. Thankfully, in most cases of interest most particle species are well coupled and are in equilibrium. In order to simplify the right hand side of (2.6) we make some standard [19] simplifying assumptions: the final states 3,4 are SM particles that have additional interactions keeping them in kinetic and chemical equilibrium and thus $f_{3,4} \rightarrow f_{3,4}^{\mathrm{eq}}$, for each state $T \ll E-\mu$ so that the $f$ follow a Maxwell-Boltzmann distribution and $1 \pm f \sim 1$, also we will focus on the case of DM being its own antiparticle i.e. $n_{1}=n_{2}$. We are left with

$$
\dot{n}+3 H n=-\int \prod_{i=1}^{4} d P_{i}(2 \pi)^{4} \delta^{(4)}\left(p_{1}+p_{2}-p_{3}-p_{4}\right)\left(f_{1} f_{2}\left|\mathscr{M}_{12,34}\right|^{2}-f_{3}^{\mathrm{eq}} f_{4}^{\mathrm{eq}}\left|\mathscr{M}_{34,12}\right|^{2}\right) .
$$

The matrix elements that appear here are just those that appear in a standard calculation of a scattering cross section,

$$
\int \prod_{i=3}^{4} d P_{i}(2 \pi)^{4} \delta^{(4)}\left(p_{1}+p_{2}-p_{3}-p_{4}\right)\left|\mathscr{M}_{12,34}\right|^{2}=4 \sqrt{\left(p_{1} \cdot p_{2}\right)^{2}-\left(m_{1} m_{2}\right)^{2}} \sigma_{12,34},
$$

allowing us to relate the right hand side of (2.7) to a cross section,

$$
\dot{n}+3 H n=-\int \frac{d^{3} p_{1}}{(2 \pi)^{3}} \frac{d^{3} p_{2}}{(2 \pi)^{3}} f_{1} f_{2}\left(\sigma v_{\mathrm{Moll}}\right)_{12 \rightarrow 34}+\int \frac{d^{3} p_{3}}{(2 \pi)^{3}} \frac{d^{3} p_{4}}{(2 \pi)^{3}} f_{3}^{\mathrm{eq}} f_{4}^{\mathrm{eq}}\left(\sigma v_{\mathrm{Moll}}\right)_{34 \rightarrow 12}
$$

where the Møller velocity is

$$
\left(v_{\text {Moll }}\right)_{i j}=\frac{\sqrt{\left(p_{i} \cdot p_{j}\right)^{2}-\left(m_{i} m_{j}\right)^{2}}}{E_{i} E_{j}}=\sqrt{\left|\frac{\vec{p}_{i}}{E_{i}}-\frac{\vec{p}_{j}}{E_{j}}\right|^{2}-\left|\frac{\vec{p}_{i}}{E_{i}} \times \frac{\vec{p}_{i}}{E_{i}}\right|^{2}} .
$$

There is little difference between the Møller velocity and the relative velocity when the particles are non-relativistic [20], but the distinction can be important at larger speeds. Furthermore, the Møller velocity is necessary to make the result Lorentz invariant. The principle of detailed balance tells us that when the initial state is in equilibrium with the final state $f_{1}^{\mathrm{eq}} f_{2}^{\mathrm{eq}}(\sigma v)_{12,34}=f_{3}^{\mathrm{eq}} f_{4}^{\mathrm{eq}}(\sigma v)_{34,12}$, allowing us to express (2.9) entirely in terms of initial state quantities. In addition, we introduce a thermally average cross section,

$$
\langle\sigma v\rangle=\frac{\int \frac{d^{3} p_{1}}{(2 \pi)^{3}} \frac{d^{3} p_{2}}{(2 \pi)^{3}} f_{1}^{\mathrm{eq}} f_{2}^{\mathrm{eq}}\left(\sigma v_{\mathrm{Moll}}\right)}{\int \frac{d^{3} p_{1}}{(2 \pi)^{3}} \frac{d^{3} p_{2}}{(2 \pi)^{3}} f_{1}^{\mathrm{eq}} f_{2}^{\mathrm{eq}}} .
$$

The utility of this thermally averaged cross section becomes apparent once we realise that even if number changing processes are slow and species have fallen out of chemical equilibrium, number 
conserving (but momentum exchanging) processes can still proceed and keep particles in kinetic equilibrium. Thus, we expect the momentum dependence of $f$ to be the same as for $f^{\text {eq }}$ i.e. $f$ is separable, $f=e^{\tilde{\mu}(t) / T} f^{\mathrm{eq}}(p, t)$. Finally, we reach the Boltzmann equation in its common form,

$$
\dot{n}+3 H n=\langle\sigma v\rangle\left(n_{\mathrm{eq}}^{2}-n^{2}\right) .
$$

Now that we have "derived" the Boltzmann equation it is time to solve it. First notice that the equation describes a competition between the rate of collisions $n\langle\sigma v\rangle$ and the rate of expansion $H$. If the scattering rate is fast then $n$ follows its equilibrium distribution $n_{\mathrm{eq}} \sim T^{3}$ for radiation and $n_{\mathrm{eq}} \sim(m T)^{3 / 2} e^{-m / T}$ for matter. Once the scattering rate drops below the expansion rate the particle can no longer stay in equilibrium and the evolution is driven by expansion, $n \sim a^{-3}$. This process of the particle falling out of thermal equilibrium is called "freeze out". At very early times when $T \gg m$ and the particle is in equilibrium $n_{\mathrm{eq}} \sim a^{-3}$. The entropy density, $s$, also redshifts in this way. This early- and late-time evolution leads us to define a useful quantity, the comoving number density $Y=\frac{n}{s}$. Furthermore, it is helpful to define a surrogate for time, $x=m / T$. We now specialise to the case of freeze out occurring while the universe is in a radiation dominated epoch with $g_{\star}$ degrees of freedom in the bath, and

$$
H_{R D}^{2}=\frac{8 \pi^{3}}{90} \frac{g_{\star} T^{4}}{m_{\mathrm{Pl}}^{2}}
$$

From (2.13) we learn that $d x / d t=\left(8 \pi^{3} g_{\star} /\left(90 m_{\mathrm{Pl}}^{2}\right)\right)^{1 / 2} m^{2} / x$, allowing us to rewrite (2.12) as

$$
\frac{d Y}{d x}=\frac{\langle\sigma v\rangle x s}{\sqrt{\frac{8 \pi^{3} g_{\star}}{90 m_{\mathrm{Pl}}^{2}}} m^{2}}\left(Y_{\mathrm{eq}}^{2}-Y^{2}\right) .
$$

Note that $s \sim x^{-3}$ and that, although suppressed in the expression, $g_{\star}$ is a function of $x$ since the number of relativistic degrees of freedom in the bath depends upon temperature. Solving this equation exactly is done numerically, see Figure 3 for a graphical rendition of the solution. But we can gain some insight by approximately solving it. Once we determine when freeze out occurs, $x_{f}$, and the ratio of $n / s$ at that time i.e. $Y\left(x_{f}\right)$ then since $Y$ is approximately constant from that point on we can determine the present day abundance of DM.

More precisely, the fraction of the critical density, $\rho_{\mathrm{cr}}$, that is made up from DM at the present time (denoted by the subscript 0 ) is

$$
\Omega h^{2}=\frac{Y_{0} s_{0} m}{\rho_{\mathrm{cr}}} h^{2} \approx \frac{Y_{f} s_{0} m h^{2}}{\rho_{\mathrm{cr}}} \approx 0.3\left(\frac{m}{\mathrm{eV}}\right) Y_{f}
$$

where $\rho_{\mathrm{cr}}=3 H_{0}^{2} m_{\mathrm{Pl}}^{2} / 8 \pi \approx 8 \times 10^{-47} h^{2} \mathrm{GeV}^{4}$ and $s_{0} \approx 2970 \mathrm{~cm}^{-3}$. The freeze out temperature is approximately determined by,

$$
n_{f}\langle\sigma v\rangle \approx H_{f} .
$$

Solving (approximately) for $n_{f}$, assuming radiation domination $H_{f} \sim T^{2} / m_{\mathrm{Pl}}$, and inserting into (2.15) we find that

$$
\Omega h^{2} \approx 0.1\left(\frac{x_{f}}{25}\right)\left(\frac{g_{\star}}{80}\right)^{-1}\left(\frac{3 \times 10^{-26} \mathrm{~cm}^{3} \mathrm{~s}^{-1}}{\langle\sigma v\rangle}\right) .
$$




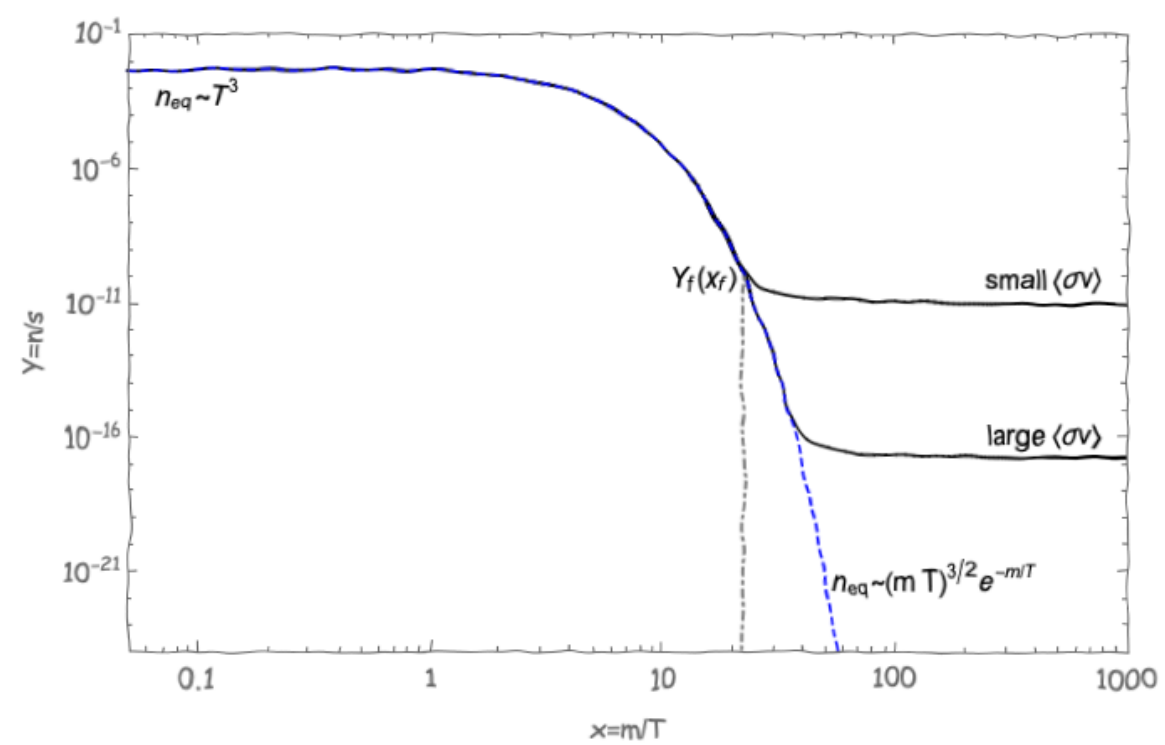

Figure 3: Cartoon of solution to Boltzmann equation. To paraphrase Christopher Marlowe, this is "the plot that launched a thousand papers".

So, the correct relic abundance is achieved for a weak-scale cross section, $\langle\sigma v\rangle \sim \alpha_{W}^{2} / M_{W}^{2} \sim 10^{-26}$ $\mathrm{cm}^{3} \mathrm{~s}^{-1}$. As we will see, for cold relics with weak-scale annihilation cross sections, $x_{f} \sim 20$ for a broad range of masses. Notice, however, that all that is required is that $\alpha^{2} / M^{2}$ is weak scale. If there are new interactions with light mediators and small couplings whose coupling to mass ratio is similar to the SM's they too could produce a thermal relic with the right abundance to be DM.

\subsection{Hot relic}

The first thing to notice about this result is that if DM freezes out when it is relativistic, then $Y_{f}$ is just the ratio of the number of DM degrees of freedom to the SM degrees of freedom at the time of freeze out. Since $g_{\star}^{\text {SM }} \lesssim 100$ if DM freezes out while relativistic it overcloses the universe if $m \gtrsim 1 \mathrm{keV}$.

\subsection{Cold relic}

An alternative way to make $Y_{f}$ small, and thus allow heavier DM, is to make it non-relativistic at the time of freeze out. We can approximately solve (2.14) under these assumptions. Freeze out occurs when $n_{\mathrm{eq}}\langle\sigma v\rangle \sim H_{f}$ i.e.

$$
x_{f}^{1 / 2} e^{-x_{f}} \approx \sqrt{\frac{8 \pi^{3} g_{\star}}{90}} \frac{1}{m_{\chi} m_{\mathrm{Pl}}\langle\sigma v\rangle} \approx \frac{1}{m_{\chi} m_{\mathrm{Pl}}\langle\sigma v\rangle}
$$

which we can solve iteratively,

$$
x_{f} \approx \log \left(m_{\chi} m_{\mathrm{P} 1}\langle\sigma v\rangle\right)+\log \log \left(m_{\chi} m_{\mathrm{P} 1}\langle\sigma v\rangle\right)+\ldots
$$

Inserting numbers we see the promised result that for a weak scale cross section $x_{f} \sim 25$. 
Question 2. Freeze out for the baryons If the baryons were a thermal relic, what would be their abundance? Estimate the cross section that keeps them in equilibrium and determine the freeze out temperature, by solving $n\langle\sigma v\rangle \approx 3 H$. Compare $\eta=n_{B} / s$ to the observed value of $\eta \sim 6 \times 10^{-10}$. Explain.

\subsection{WIMP mass range}

We have seen that annihilation cross sections near the weak scale, $\langle\sigma v\rangle \sim 10^{-26} \mathrm{~cm}^{3} \mathrm{~s}^{-1}$, can give the observed DM abundance, but for what DM masses? The lower limit on its mass comes from the Lee-Weinberg bound [21]. If DM annihilates through the weak interactions then $\langle\sigma v\rangle \sim G_{F}^{2} m_{\chi}^{2}$. Inserting this into (2.17) we see that there is too much DM unless $m \gtrsim 2 \mathrm{GeV}$. For larger masses the cross section is large enough that DM stays in equilibrium long enough that it is sufficiently depleted in the early universe. To achieve the correct abundance there must be some small couplings arising in the DM's coupling to the weak gauge bosons, for instance if the DM is an admixture of a state charged under $S U(2)$ and a SM singlet.

The upper limit on the mass is due to so-called unitarity limits, which basically boil down to the statement that for heavy enough DM mass there is only one scale in the problem, namely $m_{\chi}$. For point-like DM annihilating in the s-wave the annihilation cross section is bounded by unitarity [22],

$$
\langle\sigma v\rangle \lesssim \frac{4 \pi}{m_{\chi}^{2}} .
$$

Again, inserting this into (2.17) we see there is an upper limit of $m_{\chi} \lesssim 100 \mathrm{TeV}$, for heavier masses the annihilation cross section is too small to deplete enough of the DM.

Question 3. Annihilation cross section expanded in $x$ The thermally averaged annihilation cross section is ofter written as an expansion in relative velocity, since freeze out typically occurs when $v \approx \mathscr{O}(0.1)$ c. Show this is

$$
\left\langle\sigma v_{r e l}\right\rangle=a+b\left\langle v_{r e l}^{2}\right\rangle+\ldots=a+\frac{6 b}{x}+\ldots
$$

\subsection{Summary of WIMPs}

Summarising the previous section: a WIMP is a DM candidate which was produced thermally in the early universe. If it has weak scale or smaller interactions it can have the correct present day abundance due to its annihilation into SM states freezing out at the correct temperature, $T_{f} \approx m / 20$. This remarkably simple story works for DM in the mass range

$$
2 \mathrm{GeV} \lesssim m_{\text {WIMP }} \lesssim 100 \mathrm{TeV} .
$$

Thus, a weak scale (or smaller) annihilation cross section and a weak-scale mass leads to a thermal relic DM candidate. Going to larger masses requires DM to be composite state with a geometric annihilation cross section. Going to smaller masses requires new interactions for DM that allow it to annihilate with a cross section $\langle\sigma v\rangle \sim g_{D}^{4} m_{\chi}^{2} / m_{D}^{4}$ that is larger than the weak cross section. Such scenarios will be discussed at TASI by Tongyan Lin. 
The WIMP paradigm has been the focus of the DM field for many years, although this is now changing. One reason is in many models of BSM physics, motivated by other concerns e.g. the hierarchy problem, there is often a weak scale particle with the right properties to be WIMP DM. An obvious question is why should a new $100 \mathrm{GeV}$ particle coupled to the SM be stable? In the SM the particles that are stable are the lightest particle charged under a symmetry $e . g$. the proton is the lightest baryon and the electron is the lightest electromagnetically charged particle. In the aforementioned new physics models the WIMP candidate is the lightest particle charged under a new symmetry. The simplest example is a $\mathbb{Z}_{2}$ under which the SM is even and the BSM states are odd, thus the lightest Parity Odd Particle (LPOP) will be stable. We will now discuss in some detail one of the quintessential examples of this - supersymmetry - before returning to various other aspects of WIMP DM, which we will discuss through the lens of supersymmetry.

\section{Supersymmetry}

The prototypical example of a DM WIMP is the neutralino in the MSSM. I will now spend sometime discussing supersymmetry and the MSSM before focussing on WIMP DM within the MSSM. In Section 3.1 I will describe some problems with the SM that motivate much of BSM physics and briefly explain how SUSY deals with them. In Section 3.3 I will explain the modern language of SUSY, superfields and superspace, and construct simple supersymmetric Lagrangians. In Section 4 I will describe the field content and some features of the minimal supersymmetric version of the SM, called the MSSM, in the case where supersymmetry is unbroken. In the following section, Section 4.1, I will discuss the MSSM once SUSY is no longer an exact symmetry of the Lagrangian, using the language of spurions. I finish by discussing the spectra of the superpartners in Section 4.3. The long history of SUSY means there are many review articles and textbooks available from which to learn. There have been many previous TASI lectures on various aspects of SUSY, see Refs. [23, 24, 25, 26]. The definitive work on most aspects of this subject is the time-less "Primer" by Stephen Martin [27]. For the collider phenomenology of supersymmetric theories see previous TASI lecture notes e.g. [28, 29], or the text book by Baer and Tata [30]. On the more formal side of things the text book by Wess and Bagger [31] is an excellent resource.

There are many reasons to study supersymmetry, ranging from the formal to the practical; how one weighs each motivation depends on one's taste. The most pragmatic of them is that SUSY acts as a sort of Esperanto [32] for BSM physics ${ }^{5}$. Many phenomena that are present in SUSY are also present in other models of new physics, and any new physics discovered at the LHC will undoubtably be attributed to some variant of SUSY. The language of supersymmetry is the de facto language of most collider searches for BSM physics. It is important for experimentalists and theorists alike to be well versed in the features of SUSY. Just like the ability to converse in one foreign language often aides the ability to learn another, the understanding of SUSY will aide the understanding of much of BSM physics.

The historical discovery of SUSY serves as a valuable lesson in the power of "no-go" theorems. The theorem in question is due to Coleman and Mandula [33] and, stated loosely, says that under a set of physically reasonable assumptions (e.g. a local, relativistic field theory) the Liealgebra under which the S-matrix is symmetric is at most the direct product of the Poincare group

\footnotetext{
${ }^{5}$ I leave it up to the reader to study the history of Esperanto and to decide how hard to push this analogy.
} 
and the compact Lie group associated with internal symmetries. The major assumption, whose weakening allows for supersymmetry, is that Lie algebras are defined by commutation relations. If we allow for anti-commutation as well as commutation relations (i.e. the generators are no longer bosonic but may also be fermionic) we have graded Lie algebras and may avoid the Coleman Mandula theorem. This more general analysis was carried out by Haag, Lopuszanski and Sohnius[34] and they identified the most general graded Lie algebra allowed: the super-Poincare algebra.

The fact that supersymmetry is the most general space-time symmetry allowed by nature does not in principle mean it exists in nature, but it is a compelling reason to study it. SUSY involves introducing fermionic group generators, $Q$, and thus the action of the group, $Q|\psi\rangle=\left|\psi^{\prime}\right\rangle$, must change the spin of the state. Thus, in a supersymmetric world a bosonic state has a fermionic partner and vice versa. As we will see shortly, $Q$ commutes with the Hamiltonian so these partners are degenerate in mass. Obviously this symmetry is broken in nature. What makes us believe SUSY is something we may be able to test at weak scale experiments rather than something that is broken at some high scale like the GUT scale? There are several reasons to think that SUSY may have something to do with the $\mathrm{TeV}$ scale and we will expound on these in more detail in these lectures.

\subsection{The Hierarchy problem}

As is well known the SM suffers from the hierarchy problem - the Higgs boson is quadratically sensitive to high scale physics. Since this is one of the main motivations for SUSY to show up at the LHC it is worth discussing the issue, and how SUSY alleviates this problem, in some detail even before we have a complete definition of what SUSY is.

The Higgs boson is the only fundamental scalar in the SM and so behaves differently from all the other fields under quantum corrections. As a simple toy model consider a theory with a scalar (the Higgs) coupled to a heavy fermion (the top quark), for now we will ignore all gauge interactions. In the SM the fermion mass is generated from the scalar vev, here we will just insert it by hand. The Lagrangian is

$$
\mathscr{L}=\left|\partial_{\mu} \phi\right|^{2}+\bar{\psi} i \not \partial \psi-m_{f} \bar{\psi} \psi-y \phi \bar{\psi} \psi-\mu^{2}|\phi|^{2}-\lambda|\phi|^{4},
$$

where $\mu^{2}$ is positive. Classically there is a fermion of mass $m_{f}$ and a scalar of mass $m_{s}^{2}=\mu^{2}$. At loop level the fermion mass term and the scalar mass term receive corrections from diagrams shown in Figure 4,

$$
\begin{aligned}
\Delta m_{f} & \sim-\frac{y^{2}}{16 \pi^{2}} m_{f} \log \left(\frac{\Lambda}{m_{f}}\right), \\
\Delta \mu^{2} & \sim \frac{\lambda-y^{2}}{16 \pi^{2}} \Lambda^{2} .
\end{aligned}
$$

They differ in one very significant way, the fermion mass corrections are multiplicatively renormalised whereas the scalars have an additive renormalisation. Thus, if the tree-level fermion masses are small they remain so after quantum corrections, whereas the scalar masses are dragged up to the cutoff scale of the theory. As expected in effective field theory (EFT), all operators allowed by symmetry are generated at the cutoff scale with $\mathscr{O}(1)$ coefficients. Here the symmetry protecting 


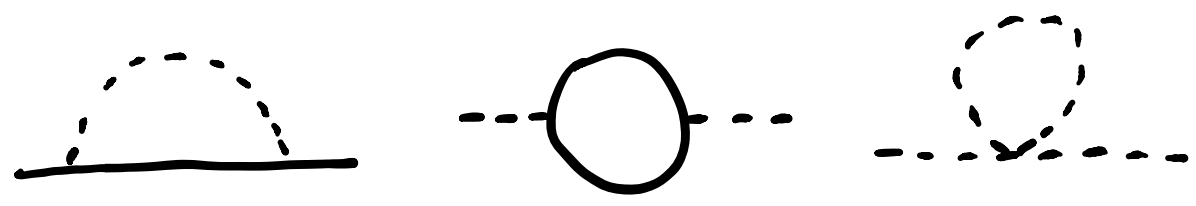

Figure 4: One loop corrections to fermions and scalars.

the fermion mass is a chiral symmetry, $\psi \rightarrow e^{i \alpha \gamma_{5}} \psi$. This is broken by the mass term and results in the loop correction being proportional to $m_{f}$. There is no such symmetry for the scalar. If the scalar were related to the fermion through a symmetry then the quadratic divergence would be removed, since it doesn't exist for the fermion. In a supersymmetric world where $\psi$ and $\phi$ are related by supersymmetry we would find that $\lambda$ and $y$ are related, leading to the necessary cancellation.

By supersymmetrizing the SM the quadratic divergence of the Higgs mass can be cutoff, this provides one motivation for the introduction of SUSY. The Higgs is responsible for electroweak symmetry breaking, which is associated with the $\sim 100 \mathrm{GeV}$ scale, and in a natural theory this is the mass we would expect for the Higgs. We see from (3.2) that there are large quantum corrections to any bare mass the Higgs may have. If the SM is an effective theory up to high scales, for instance the GUT scale $\sim 10^{16} \mathrm{GeV}$, then there will be large one-loop corrections to its mass. To maintain the physical mass to be $\sim 100 \mathrm{GeV}$ there will need to be large cancellations between the bare mass and the quantum corrections. If instead the SM becomes supersymmetric at some scale $\Lambda_{S U S Y}$, i.e. above this scale there are superpartners of the SM fields present in the theory, these quadratic divergences will be cutoff. Requiring that there is only an $\mathscr{O}(1)$ tuning between the bare mass and the quantum corrections, cutoff at the scale $\Lambda_{S U S Y}$, we expect the superpartners to enter the theory around $4 \pi \times m_{H} \sim \mathrm{TeV}$.

\subsection{Gauge coupling unification}

The gauge couplings of the SM depend on energy in a way determined by the renormalization group equations (RGEs). If one assumes that there are no new states above the weak scale, a so called desert, the three gauge couplings run in such a way that they are nearly all the same value at a high scale, $\sim 10^{14} \mathrm{GeV}$. This remarkable fact, that three a priori independent parameters have the same value at high scales is suggestive: perhaps $S U(3) \times S U(2) \times U(1)$ of the SM are really three pieces of one larger unified group, e.g. $S U(5)$ or $S O(10)$, that is broken at the high scale. This idea, and the models that realise it, are called GUTs, Grand Unified Theories.

However, the unification is far from perfect in the SM. Although the three lines do get close to one another at a high scale the unification is not ideal, and the scale of closest approach is low enough that proton decay, mediated by gauge bosons at the GUT scale which are left over when the GUT group is broken, should already have been observed. In the MSSM there are additional states at, and just above, the weak scale that will alter the RGEs and the running of the gauge couplings. Assuming that they are the only new states, i.e. there is a SUSY desert, one can calculate the gauge coupling running. Remarkably, the couplings now unify to a far greater degree and at a higher scale, $\sim 10^{16} \mathrm{GeV}$, than before, correcting both of the problems of the SM. Figure 5 shows an illustration of the the gauge coupling running, at one loop, in both the SM and the MSSM. 


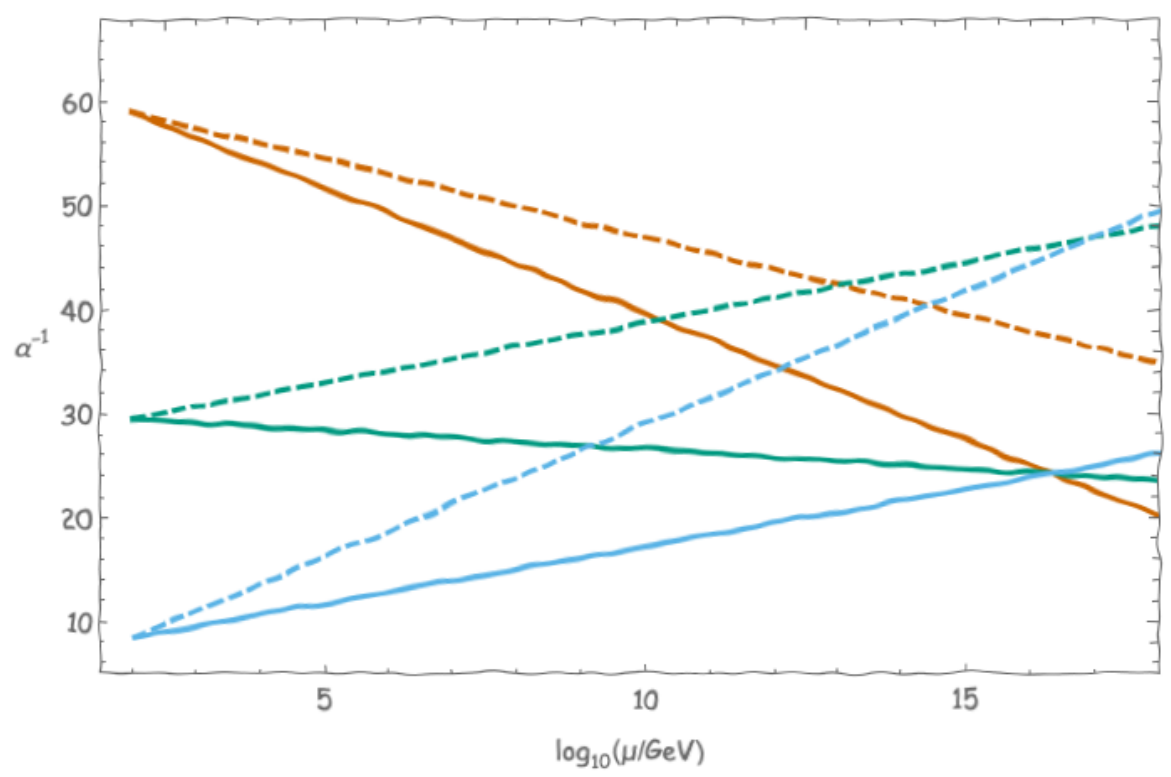

Figure 5: One loop gauge coupling evolution for the SM (dashed lines) and the MSSM (solid lines). The $S U$ (3) gauge coupling is shown in blue (bottom lines), the $S U(2)$ in green (middle lines) and the $U(1)$, in GUT normalisation $\left(g_{1}=\sqrt{5 / 3} g^{\prime}\right)$, in orange (top lines).

\subsection{Superfield (and other) formalism}

“... what he needed was a notion, not a notation."

- Gauss writing about the mathematician John Wilson

In this section I will attempt to explain all the formalism necessary to understand the remainder of the lectures. Although it is not necessary to understand the superfield formalism to learn supersymmetry, it is the language used by most practitioners and is well worth the effort to learn. There are many other places one can look to learn the formalism, but you should be aware that they almost all use different notations and conventions, both from these lectures and each other.

I will use the "West Coast" metric, $g_{\mu \nu}=\eta_{\mu \nu}=\operatorname{diag}(1,-1,-1,-1)$. When one first learns field theory fermions are introduced using Dirac spinors, $\Psi_{D}$. In supersymmetric field theories it is convenient to instead use Weyl spinors. For a detailed analysis of how they are related see Ref. [35]. Dirac spinors are, in 4 dimensions, 4 component objects while Weyl spinors are 2 component. By working in the Weyl, or chiral, basis for the $\gamma$-matrices the relationships between the two become transparent:

$$
\begin{gathered}
\gamma^{\mu}=\left(\begin{array}{cc}
0 & \sigma^{\mu} \\
\bar{\sigma}^{\mu} & 0
\end{array}\right) \quad \gamma_{5}=\left(\begin{array}{cc}
-1 & 0 \\
0 & 1
\end{array}\right) \\
\sigma^{\mu}=(1, \vec{\sigma}), \quad \bar{\sigma}^{\mu}=(1,-\vec{\sigma})
\end{gathered}
$$

with

$$
\sigma^{1}=\left(\begin{array}{ll}
0 & 1 \\
1 & 0
\end{array}\right), \sigma^{2}=\left(\begin{array}{cc}
0 & -i \\
i & 0
\end{array}\right), \sigma^{3}=\left(\begin{array}{cc}
1 & 0 \\
0 & -1
\end{array}\right)
$$


Recall also the combination $\sigma^{\mu \nu}=\frac{i}{4}\left(\sigma^{\mu} \bar{\sigma}^{v}-\sigma^{v} \bar{\sigma}^{\mu}\right)$. The Dirac spinor may be built from a lefthanded and right-handed Weyl spinor. In SUSY, and much of BSM physics, it is useful to work with only left-handed spinors. Recalling that right-handed spinors are hermitian conjugates of left-handed fields,

$$
\Psi_{D}=\left(\begin{array}{c}
\chi \\
\eta^{\dagger}
\end{array}\right),
$$

where both $\chi$ and $\eta$ are left-handed. Until now I have suppressed indices, and will do so for most of the rest of the lectures, but occasional it will be necessary to include them. With indices attached (3.6) becomes,

$$
\Psi_{D}=\left(\begin{array}{c}
\chi_{\alpha} \\
\eta^{\dagger \dot{\alpha}}
\end{array}\right) .
$$

The indices are raised and lowered with $\varepsilon_{\alpha \beta}$ and $\varepsilon^{\alpha \beta}$ with $\varepsilon^{12}=-\varepsilon^{21}=\varepsilon_{21}=-\varepsilon_{12}=1$, all others 0 . Spinor summations are defined as

$$
\chi \eta \equiv \chi^{\alpha} \eta_{\alpha}, \chi^{\dagger} \eta^{\dagger} \equiv \chi_{\dot{\alpha}}^{\dagger} \eta^{\dagger \dot{\alpha}} .
$$

Once these spinor summation conventions are defined we can usually get away with suppressing the indices.

Question 4. Show $\chi \eta=\eta \chi$.

\subsection{Superspace}

With the addition of supersymmetry the usual algebra of the Lorentz group is extended by the supersymmetry algebra which, for $N=1$ supersymmetry in 4 dimensions, is

$$
\begin{array}{r}
\left\{Q_{\alpha}, Q_{\dot{\beta}}^{\dagger}\right\}=2 \sigma_{\alpha \dot{\beta}}^{\mu} P_{\mu} \\
\left\{Q_{\alpha}, Q_{\beta}\right\}=\left\{Q_{\dot{\alpha}}^{\dagger}, Q_{\dot{\beta}}^{\dagger}\right\}=0 \\
{\left[P_{\mu}, Q_{\alpha}\right]=\left[P_{\mu}, Q_{\dot{\alpha}}^{\dagger}\right]=0}
\end{array}
$$

The generators of the SUSY algebra, $Q_{\alpha}$ are spinors and SUSY transformations are of the form boson $\leftrightarrow$ fermion. Equation (3.11) indicates that SUSY transformations commute with the Hamiltonian and states related by a SUSY transformation have the same mass, such states are called superpartners. From (3.9) we see that two SUSY transformations amount to a spacetime translation i.e. supersymmetry is a spacetime symmetry. This suggests the concept of superspace, augmenting the usual four (commuting) coordinates $x^{\mu}$ to include 4 anticommuting (Grassmann) coordinates $\theta_{\alpha}, \bar{\theta}_{\dot{\alpha}} \equiv\left(\theta_{\alpha}\right)^{\dagger}$. Recall the features of Grassmann spinors:

$$
\left\{\theta^{\alpha}, \theta^{\beta}\right\}=\left\{\bar{\theta}_{\dot{\alpha}}, \bar{\theta}_{\dot{\beta}}\right\}=\left\{\theta^{\alpha}, \bar{\theta}_{\dot{\beta}}\right\}=0,
$$

leading to the result that the square of a Grassmann coordinate is zero, making for simple Taylor series. For Grassmann variables integration is akin to differentiation and,

$$
\int d^{2} \theta \theta^{2} \equiv \int d^{2} \theta \theta^{\alpha} \theta_{\alpha}=1 \int d^{2} \theta d^{2} \bar{\theta} \theta^{2} \bar{\theta}^{2}=1
$$


Question 5. Show $d^{2} \theta=-\frac{1}{4} d \theta^{\alpha} d \theta^{\beta} \varepsilon_{\alpha \beta}$ and $\frac{\partial^{2}}{\partial \theta^{\alpha} \partial \theta_{\alpha}} \theta^{2}=4$.

Just as the momentum operator, $-i \partial_{\mu}$, is the generator of space-time translations we would like to determine the generator, $Q_{\alpha}$, of SUSY transformations. An obvious guess is $Q_{\alpha}=-i \frac{\partial}{\partial \theta^{\alpha}}$ but it is easy to check that this does not satisfy the algebra of (3.9). Instead the generators are,

$$
\begin{aligned}
& Q_{\alpha}=\frac{\partial}{\partial \theta^{\alpha}}-i \sigma_{\alpha \dot{\beta}}^{\mu} \bar{\theta}^{\dot{\beta}} \partial_{\mu} \\
& \bar{Q}_{\dot{\alpha}}=\frac{\partial}{\partial \bar{\theta}^{\dot{\alpha}}}-i \theta^{\beta} \sigma_{\beta \dot{\alpha}}^{\mu} \partial_{\mu}
\end{aligned}
$$

Question 6. Show that these $Q$ do indeed satisfy the SUSY algebra.

With the generators in hand we may exponentiate and carry out a finite SUSY transformation on a function of superspace, which has a remarkably simple form.

Question 7. Confirm that

$$
e^{\varepsilon Q+\bar{\varepsilon} \bar{Q}} f\left(x^{\mu}, \theta, \bar{\theta}\right)=f\left(x^{\mu}+i \varepsilon \sigma^{\mu} \bar{\theta}+i \theta \sigma^{\mu} \bar{\varepsilon}, \theta+\varepsilon, \bar{\theta}+\bar{\varepsilon}\right) .
$$

The final piece we need to introduce are the superspace derivatives, which anti-commute with the generators and are given by ${ }^{6}$

$$
\begin{aligned}
D_{\alpha} & =\frac{\partial}{\partial \theta^{\alpha}}+i\left(\sigma^{\mu} \bar{\theta}\right)_{\alpha} \partial_{\mu} \\
\bar{D}_{\dot{\alpha}} & =-\frac{\partial}{\partial \bar{\theta}^{\dot{\alpha}}}-i\left(\theta \sigma^{\mu}\right)_{\dot{\alpha}} \partial_{\mu}
\end{aligned}
$$

So far this may seem like formality for formality's sake, but its utility will hopefully soon become very clear. Rather than working with component fields, e.g. fermions and scalars, and constructing Lagrangians that must be painstakingly checked to ensure SUSY is preserved we can instead work with superfields and supersymmetry is ensured. It is much like using four vectors in relativity, if there are no "hanging indices" then Lorentz invariance is maintained without having to worry about how $t, x, y$, and $z$ transform under a particular boost. In addition, actions are now built from integrals over superspace, $\int d^{4} x d^{2} \theta d^{2} \bar{\theta}$.

Thanks to the properties of Grassmann coordinates (3.12) the most general superfield can be Taylor expanded in its $\theta$ coordinates.

$$
G(x, \theta, \bar{\theta})=\phi(x)+\theta \psi+\bar{\theta} \bar{\chi}+\theta^{2} m+\bar{\theta}^{2} n+\theta \sigma^{\mu} \bar{\theta} V_{\mu}+\theta^{2} \bar{\theta} \bar{\lambda}+\bar{\theta}^{2} \theta \rho+\theta^{2} \bar{\theta}^{2} d
$$

This is a lot of fields, more than we would expect to realise supersymmetry given the toy example discussed in the introduction. This general representation (3.20) is reducible, and by imposing constraints we can build smaller irreducible representations. It is these we will use to describe supersymmetric field theories.

\footnotetext{
${ }^{6}$ Notice that I have (deliberately) started to become more sloppy with indices, but there is still enough information to replace them all, should you feel so inclined.
} 


\begin{tabular}{c|c|c} 
Field & Off-shell & On-shell \\
\hline$\phi$ & 2 & 2 \\
$\psi$ & 4 & 2 \\
$F$ & 2 & 0
\end{tabular}

Table 1: Number of degrees of freedom of components of the chiral multiplet.

\subsection{Chiral superfield}

We can build a smaller representation, the chiral superfield, by imposing the constraint

$$
\bar{D} \Phi=0 .
$$

Notice that since $\{D, Q\}=0$ this constraint is invariant under SUSY transformations. To identify what a chiral superfield is in terms of components first note that

$$
\bar{D}_{\dot{\alpha}}\left(x^{\mu}+i \theta \sigma^{\mu} \bar{\theta}\right)=0 \quad \text { and } \quad \bar{D}_{\dot{\alpha}} \theta=0 .
$$

Thus, a chiral superfield is a function of $y=x^{\mu}+i \theta \sigma^{\mu} \bar{\theta}$ and $\theta$. Then, expanding as before in powers of $\theta$,

$$
\begin{aligned}
\Phi(y, \theta)= & \phi(y)+\sqrt{2} \theta \psi(y)+\theta^{2} F(y) \\
= & \phi(x)-i \theta \sigma^{\mu} \bar{\theta} \partial_{\mu} \phi-\frac{1}{4} \theta^{2} \bar{\theta}^{2} \partial^{2} \phi \\
& +\sqrt{2} \theta \psi+\frac{i}{\sqrt{2}} \theta^{2} \partial_{\mu} \psi \sigma^{\mu} \bar{\theta}+\theta^{2} F .
\end{aligned}
$$

So we see that the chiral superfield contains a complex scalar, $\phi$, a Weyl fermion, $\psi$ and another complex scalar, $F$, that we will refer to as an auxiliary field (we will see why shortly). It is the perfect candidate to use for the matter and Higgs fields in a supersymmetric version of the SM. Note also that any analytic function of chiral superfields (i.e. a function made out of powers of $\Phi$ and no powers of $\Phi^{\dagger}$ ) is itself a chiral superfield.

Question 8. Using the results of the previous exercise work out the SUSY transformations on the components of the chiral superfield. That is, calculate $\delta \Phi=(\varepsilon Q+\bar{\varepsilon} \bar{Q}) \Phi$ and confirm that,

$$
\delta \phi=\sqrt{2} \varepsilon \psi, \quad \delta \psi=\sqrt{2} \varepsilon F+\sqrt{2} i \sigma^{\mu} \bar{\varepsilon} \partial_{\mu} \phi, \quad \delta F=i \sqrt{2} \bar{\varepsilon} \sigma^{\mu} \partial_{\mu} \psi .
$$

Chiral superfields can be combined in various ways to build superspace, and therefore supersymmetric, invariants. From (3.21) we see that any holomorphic function of chiral superfields is itself a chiral superfield. Also, notice that the highest component of the chiral superfield transforms into a total derivative under a SUSY transformation [see the previous exercise (3.25)]. This is true for the highest component of any supermultiplet and is as expected on dimensional grounds; since $F$ is the highest dimension field in the multiplet and the SUSY transformation involves $\varepsilon$ whose dimension is $[\varepsilon]=-1 / 2$, making up the units requires a derivative. Since any holomorphic function of chiral superfields is itself a chiral superfield, then the quantity

$$
\int d^{4} x \int d^{2} \theta W(\Phi)
$$


where $W$ is a polynomial in $\Phi$, is a SUSY invariant and a perfect candidate for a term in a SUSY action. Thus, for chiral superfields an integral over half of superspace is invariant. Alternatively, $\bar{\theta}^{2} f(\Phi)$ is invariant when integrated over all of superspace but using (3.13) this reduces to integrating over only $\theta^{2}$.

Functions of both $\Phi$ and $\Phi^{\dagger}$ must be integrated over the whole of superspace in order to be invariant. Thus, we can now write down the most general supersymmetric invariant action built from chiral superfields, $\Phi_{i}$,

$$
S=\int d^{4} x\left[\int d^{4} \theta K\left(\Phi_{i}^{\dagger}, \Phi_{j}\right)+\int d^{2} \theta W\left(\Phi_{i}\right)+\text { h.c. }\right] .
$$

$K$ is the Kähler potential and is real and $W$ is the superpotential and is holomorphic in the chiral superfield(s). The chiral superfield has dimension $[\Phi]=1$, the same as for its scalar $\phi$, which means that $[\theta]=-1 / 2$. So the $\mathrm{K}^{\prime}$ ahler potential must have dimension 2 and the superpotential dimension 3 , which will limit the renormalizable terms we can write down. Let us examine a simple example of a supersymmetric theory constructed entirely from chiral superfields. In so doing some of the formalism's utility will become apparent.

\section{Wess-Zumino model}

The most general supersymmetric, renormalizable model of a single chiral superfield has Lagrangian density

$$
\int d^{4} \theta \Phi^{\dagger} \Phi+\int d^{2} \theta\left(\frac{m}{2} \Phi^{2}+\frac{\lambda}{3} \Phi^{3}\right)+\text { h.c. }
$$

Using the results of the previous subsection we can expand the superfield in its components and find

$$
\begin{aligned}
\mathscr{L}= & \partial^{\mu} \phi^{*} \partial_{\mu} \phi+\psi^{\dagger} i \bar{\sigma}^{\mu} \partial_{\mu} \psi+F^{*} F \\
& +m F \phi-\frac{1}{2} m \psi \psi+\text { h.c. }+\lambda F \phi^{2}-\lambda \phi \psi \psi+\text { h.c. }
\end{aligned}
$$

The first line comes from the Kähler potential in (3.28) and the second from the superpotential. This looks like a model of an interacting Weyl fermion and a complex scalar very similar to that discussed in the introduction, but what about $F$ ? There is no $\partial F / \partial t$ term in the Lagrangian. It is not a propagating field so its equations of motion will be algebraic, hence the name auxiliary field. This explains the counting shown in Table 1, after application of the equations of motion the only degrees of freedom are contained in the fermion and boson and they match. But off-shell, where the equations of motion are not applied, we need to introduce additional bosonic degrees of freedom. The introduction of the auxiliary fields and of the superspace notation gives a representation of supersymmetry that closes even off-shell. The difference between the reduction in the number of off-shell and on-shell degrees of freedom for bosons and fermions is explained by the order of their equations of motion, and therefore the number of boundary conditions that have to be specified to solve them.

Since the F-term equations are algebraic in the other fields they can be solved for and reinserted into the Lagrangian. For the simple case with canonical Kähler potential, $K=\Phi^{\dagger} \Phi$, the F-term equations of motion are

$$
F^{*}=-\frac{\partial W}{\partial \phi}
$$


Inserting these equations back into the action results in a contribution to the potential from these F-terms,

$$
V_{F}=|F|^{2}=\left|\frac{\partial W}{\partial \phi}\right|^{2},
$$

notice that this potential is positive semi-definite.

Doing this for the Wess-Zumino model we find

$$
F^{*}=-\frac{\partial W}{\partial \phi}=-\left(m \phi+\lambda \phi^{2}\right)
$$

and then

$$
\mathscr{L}=\left|\partial_{\mu} \phi\right|^{2}+\psi^{\dagger} i \bar{\sigma}^{\mu} \partial_{\mu} \psi-\frac{1}{2} m \psi \psi-\lambda \phi \psi \psi+\text { h.c. }-\left|m \phi+\lambda \phi^{2}\right|^{2} .
$$

This is then a model of a fermion interacting with a scalar. They are degenerate in mass, and if you were to calculate the loop corrections to the scalar masses you would find there is no quadratic divergence. This last statement is easy to see from the example in Section 3.1, supersymmetry relates the Yukawa coupling to the scalar self coupling and the quadratic divergence of (3.2) is cancelled. The additional scalar ${ }^{3}$ coupling present in the Wess-Zumino model cannot introduce quadratic divergences in the scalar mass ${ }^{2}$ since the coupling is dimensionful. Furthermore, because the scalar and fermion masses are the same all logarithmic divergences also cancel.

For completeness, the general case, with arbitrary number of chiral superfields $\Phi_{i}$, where the Lagrangian is given by (3.27) leads to a potential

$$
V=\frac{\partial W^{*}}{\partial \phi_{i}^{*}} K_{i j}^{-1} \frac{\partial W}{\partial \phi_{j}}, \text { where } K_{i j}=\frac{\partial K}{\partial \phi_{i}^{*} \partial \phi_{j}} .
$$

\subsection{Vector superfield}

Another constraint that can be placed on the general superfield is that of reality,

$$
V^{\dagger}=V .
$$

Doing so will lead us to the vector superfield. The full vector superfield still has many components but we can take advantage of the fact that all the vectors in the SM are gauge bosons and have a related gauge symmetry ${ }^{7}, A_{\mu} \rightarrow A_{\mu}+\partial_{\mu} \Lambda$, to try to gauge some of the components away. We extend the gauge transformations to act on superfields by noticing that for a chiral superfield $\Lambda$ the combination $\Lambda+\Lambda^{\dagger}$ is real so $V+\left(\Lambda+\Lambda^{\dagger}\right)$ is still a vector superfield. In addition, both expansions contain terms that behave in the correct way to be the symmetry transformation on the gauge field,

$$
V=\ldots+\theta \sigma^{\mu} \bar{\theta} A_{\mu}+\ldots, \quad \text { and } \quad \Lambda+\Lambda^{\dagger}=\ldots+i \theta \sigma^{\mu} \bar{\theta} \partial_{\mu}\left(\phi-\phi^{\dagger}\right)+\ldots
$$

Using this gauge transformation we can write the vector superfield in the Wess-Zumino gauge where many of the components have been gauged away, leaving just a vector, a fermion and a real scalar (another auxiliary field),

$$
V \stackrel{\text { WZgauge }}{=} 2 \theta \sigma^{\mu} \bar{\theta} A_{\mu}+2 \theta^{2} \bar{\theta} \lambda^{\dagger}+2 \theta \bar{\theta}^{2} \lambda+\theta^{2} \bar{\theta}^{2} D .
$$

\footnotetext{
${ }^{7}$ For now we restrict ourselves to Abelian groups.
} 
Thus the vector multiplet contains the gauge fields and their superpartners, the gauginos. In order to write down the kinetic terms for the gauge fields and its superpartner we introduce the (gauge covariant) chiral superfield, $W_{\alpha}$, built from the vector superfield,

$$
W_{\alpha}=-\frac{1}{8} \bar{D}^{2} D_{\alpha} V, \text { and } \bar{W}_{\dot{\alpha}}=-\frac{1}{8} D^{2} \bar{D}_{\alpha} V
$$

Expanding in terms of component fields leads to,

$$
W_{\alpha}=\lambda_{\alpha}+\theta_{\alpha} D-\left(\sigma^{\mu v} \theta\right)_{\alpha} F_{\mu v}+i \theta^{2} \sigma^{\mu} \partial_{\mu} \lambda^{\dagger},
$$

and explains the often used name of supersymmetric field strength. The field strength has scaling dimension $\left[W_{\alpha}\right]=3 / 2$ and the only renormalizable operator we can build from it is a superpotential term,

$$
\begin{aligned}
& \frac{1}{8 \pi} \operatorname{Im}\left[\left(\frac{4 \pi i}{g^{2}}+\frac{\theta_{Y M}}{2 \pi}\right) \int d^{2} \theta W^{\alpha} W_{\alpha}\right] \\
= & -\frac{1}{4 g^{2}} F_{\mu v} F^{\mu v}+\frac{i}{g^{2}} \lambda^{\dagger} \bar{\sigma}^{\mu} D_{\mu} \lambda+\frac{1}{2 g^{2}} D^{2}-\frac{\theta_{Y M}}{32 \pi^{2}} F_{\mu v} \widetilde{F}^{\mu v} .
\end{aligned}
$$

Question 9. By applying the SUSY generators (3.15) to (3.37) show that restricting to the Wess-Zumino gauge breaks supersymmetry.

It is common to treat the combination of gauge coupling and $\theta$-angle as one quantity, a complex gauge coupling, $\tau=\frac{4 \pi i}{g^{2}}+\frac{\theta_{Y M}}{2 \pi}$, and for most discussions it is sufficient to assume $\theta_{Y M}=0$. In this case the Lagrangian term is,

$$
\frac{1}{4 g^{2}} \int d^{2} \theta W^{\alpha} W_{\alpha}+\text { h.c. }=-\frac{1}{4 g^{2}} F_{\mu v} F^{\mu v}+\frac{i}{g^{2}} \lambda^{\dagger} \bar{\sigma}^{\mu} D_{\mu} \lambda+\frac{1}{2 g^{2}} D^{2} .
$$

As expected the auxiliary field, $D$, has no kinetic term and again its equation of motion will be algebraic.

If the chiral superfields of the previous section are charged under the gauge group then they transform as,

$$
\Phi \rightarrow e^{-q \Lambda} \Phi,
$$

which means that the Kähler potential of (3.27) is no longer gauge invariant. Including the gauge interactions the most general Lagrangian involving vector and chiral superfields of charge $q_{i}$ becomes,

$$
\mathscr{L}=\int d^{4} \theta K\left(\Phi_{i}^{\dagger}, e^{q_{i} V} \Phi_{i}\right)+\int d^{2} \theta \tau W_{\alpha} W^{\alpha}+h . c+\int d^{2} \theta W\left(\Phi_{i}\right)+\text { h.c. }
$$

We will limit ourselves to the canonical (renormalizable) Kähler term, $K=\Phi^{\dagger} e^{q V} \Phi$ for which,

$$
\begin{aligned}
\int d^{4} \theta \Phi_{i}^{\dagger} e^{q_{i} V} \Phi_{i}= & D^{\mu} \phi_{i}^{*} D_{\mu} \phi_{i}+\psi_{i}^{\dagger} i \bar{\sigma}^{\mu} \partial_{\mu} \psi_{i}+F_{i}^{*} F_{i} \\
& +\sqrt{2} \sum_{i} q_{i}\left(\phi_{i}^{*} \psi_{i} \lambda+\lambda^{\dagger} \psi_{i}^{\dagger} \phi_{i}\right)+\sum_{i} q_{i} D \phi_{i}^{*} \phi_{i}
\end{aligned}
$$

Combining this with (3.40) we can solve for the D-term and find,

$$
D=-g^{2} \sum_{i} q_{i} \phi_{i}^{*} \phi_{i}
$$




\begin{tabular}{c|c|c} 
Field & Off-shell & On-shell \\
\hline$A_{\mu}$ & 3 & 2 \\
$\lambda$ & 4 & 2 \\
$D$ & 1 & 0
\end{tabular}

Table 2: Number of degrees of freedom of components of the vector multiplet.

As before we can remove the auxiliary field from the Lagrangian and we find that it contributes to the potential,

$$
V_{D}=\frac{1}{2} g^{2}\left(\sum_{i} q_{i} \phi_{i}^{*} \phi_{i}\right)^{2} .
$$

So far we have limited ourselves to Abelian groups. For non-Abelian groups chiral multiplets whose representation have generators $T^{a}$, transform as,

$$
\Phi \rightarrow e^{-T^{a} \Lambda^{a}} \Phi, \Phi^{\dagger} \rightarrow \Phi^{\dagger} e^{-T^{a} \Lambda^{a^{\dagger}}}
$$

in particular fundamental and anti-fundamental representations have a relative minus sign in the way they transform. The vector superfield now has a more complicated transformation,

$$
e^{T^{a} V^{a}} \rightarrow e^{T^{a} \Lambda^{a \dagger}} e^{T^{a} V^{a}} e^{T^{a} \Lambda^{a}}
$$

and the supersymmetric field strength is now,

$$
W_{\alpha}^{a} T^{a}=-\frac{1}{4} \bar{D}^{2} e^{-T^{a} \Lambda^{a}} D_{\alpha} e^{T^{a} V^{a}} .
$$

For the particular case of an Abelian group there is one more supersymmetric and gauge invariant term we can add to the Lagrangian, the Fayet-Iliopolis term,

$$
\xi \int d^{4} \theta V=\xi D
$$

which acts as a source for the D-term.

In a general theory involving chiral and vector superfields the scalar potential is given by the sum of F-term and D-term contributions,

$$
V=V_{F}+V_{D}
$$

and it is positive semi-definite, $V \geq 0$. In fact, if and only if the F-term and D-term equations can be solved $^{8}$ (i.e. $F_{i}=0$ and $D^{a}=0$ ) is supersymmetry unbroken. To see this recall the SUSY algebra (3.9) and take the expectation value of the trace of (3.9) in the vacuum,

$$
\begin{aligned}
\left\langle 0\left|4 P^{0}\right| 0\right\rangle & =\left\langle 0\left|\left\{Q_{\alpha}, Q_{\dot{\alpha}}^{\dagger}\right\}\right| 0\right\rangle=\left\langle 0\left|\left(Q_{1} Q_{1}^{\dagger}+Q_{1}^{\dagger} Q_{1}+Q_{2} Q_{2}^{\dagger}+Q_{2}^{\dagger} Q_{2}\right)\right| 0\right\rangle \\
& \left.\left.\left.\left.=\left|Q_{1}^{\dagger}\right| 0\right\rangle\left.\right|^{2}+\left|Q_{1}\right| 0\right\rangle\left.\right|^{2}+\left|Q_{2}^{\dagger}\right| 0\right\rangle\left.\right|^{2}+\left|Q_{2}\right| 0\right\rangle\left.\right|^{2} \\
& \geq 0
\end{aligned}
$$

\footnotetext{
${ }^{8}$ In non-Abelian theories the existence of a supersymmetric vacuum is determined entirely by the F-term equations.
} 
If the vacuum $|0\rangle$ is invariant under a supersymmetric transformation then $Q|0\rangle=0$ and SUSY is unbroken and the vacuum energy $\langle 0|H| 0\rangle=0$ and thus $F=0$ and $D=0$. Otherwise if SUSY is spontaneously broken ( $Q$ does not annihilate the vacuum) the vacuum energy is positive, since the right side of (3.52) is positive semi-definite, and one of the F or D-terms is non-zero.

\subsection{R-symmetry}

With the introduction of superspace coordinates it is possible to define a new symmetry of the action. Under this R-symmetry the $\theta$ coordinate picks up a phase,

$$
\theta \rightarrow e^{i \alpha} \theta, \quad \text { and } \quad \bar{\theta} \rightarrow e^{-i \alpha} \bar{\theta}
$$

From our definition of integration of Grassmann coordinates (3.13) we see $d \theta$ rotates the opposite way to $\theta$. This means that if the Kähler potential has R-charge 0 and the superpotential has Rcharge 2 the action will be R-symmetric. One immediate consequence of this is that $W_{\alpha}$ and therefore gauginos have R-charge 1 . Under an R-symmetry transformation $\theta$ rotates by a phase, so different components of a superfield must have different R-charges. As an example consider the superpotential $W=m \Phi^{2}$ which is R-symmetric if $\Phi$ has R-charge 1, its components then transform as,

$$
\phi(x) \rightarrow e^{i \alpha} \phi(x), \psi(x) \rightarrow \psi(x), F \rightarrow e^{-i \alpha} F .
$$

\subsection{Putting the formalism to work: O'Raifeartaigh and other models}

So far we have concentrated on writing down supersymmetric actions without worry about whether the ground state is supersymmetric. Now we will consider the simplest class of models that spontaneously break SUSY, and in so doing learn a few general rules about models that break SUSY at tree level and how one goes about analyzing models of SUSY breaking.

The simplest models ${ }^{9}$ that break supersymmetry are O'Raifeartaigh models [37] and are built from chiral superfields. Consider as an example the model with 3 chiral superfields, $A, B, X$ and superpotential,

$$
W=\lambda X\left(A^{2}-\mu^{2}\right)+m A B+\text { h.c. },
$$

we will assume throughout that the parameters are all real. The F-term equations are,

$$
\begin{aligned}
& F_{X}^{*}=-\frac{\partial W}{\partial X}=\lambda\left(A^{2}-\mu^{2}\right)=0 \\
& F_{A}^{*}=-\frac{\partial W}{\partial A}=m B+2 \lambda A X=0 \\
& F_{B}^{*}=-\frac{\partial W}{\partial B}=m A=0,
\end{aligned}
$$

which cannot be simultaneously solved and thus SUSY is broken. It is instructive to examine the spectrum in this model, to do so we will need the fermion, $M_{F}$, and scalar, $M_{S}^{2}$, mass matrices. At tree-level these are simply given by,

$$
\left.M_{F}\right|_{i j}=\frac{\partial^{2} W}{\partial \Phi_{i} \partial \Phi_{j}} \quad \text { and }\left.\quad M_{S}^{2}\right|_{i j}=\frac{\partial^{2} V}{\partial \phi_{i} \partial \phi_{j}}
$$

\footnotetext{
${ }^{9}$ The Poloyni model [36] has just a linear superpotential, $W=\mu^{2} Z$, and is simpler, but rather boring to analyze.
} 
For the O'Raifeartaigh model of interest the potential is given by,

$$
V=\left|F_{X}\right|^{2}+\left|F_{A}\right|^{2}+\left|F_{B}\right|^{2}=\left|\lambda\left(A^{2}-\mu^{2}\right)\right|^{2}+|m B+2 \lambda A X|^{2}+|m A|^{2},
$$

which has a flat direction since (3.57) can always be solved regardless of the values of the other fields. This vacuum degeneracy will be lifted by loop corrections. If $m^{2}-2 \lambda^{2} \mu^{2}>0$ the minimum is at the origin, otherwise $A$ acquires a vev. The two minima are

$$
\begin{aligned}
A & =0, B=0 \\
A^{2} & =\frac{2 \lambda^{2} \mu^{2}-m^{2}}{2 \lambda^{2}}, B=\frac{2 \lambda}{m} \sqrt{\frac{2 \lambda^{2} \mu^{2}-m^{2}}{2 \lambda^{2}}} X .
\end{aligned}
$$

At the first, $V=\lambda^{2} \mu^{4}$ and at the second $V=m^{2}\left(\mu^{2}-\frac{m^{2}}{4 \lambda^{2}}\right)$. Concentrating on the case with the vacuum at the origin the fermion mass matrix in the $\left(\psi_{X}, \psi_{A}, \psi_{B}\right)$ basis is given by,

$$
M_{F}=\frac{\partial^{2} W}{\partial \Phi_{i} \partial \Phi_{j}}=\left(\begin{array}{ccc}
0 & 0 & 0 \\
0 & 2 \lambda x & m \\
0 & m & 0
\end{array}\right)
$$

where $x=\langle X\rangle$. The three fermions have mass 0 , and $\lambda x \pm \sqrt{m^{2}+\lambda^{2} \lambda x^{2}}$. The massless fermion is the Goldstino, the analogue of the Goldstone boson of spontaneously broken global symmetries. Here it is fermionic since the spontaneoulsy broken symmetry is SUSY and its generators are fermionic not bosonic.

The scalar mass matrix is more complicated. In principle it is a $6 \times 6$ matrix but since $X$ and $X^{*}$ don't acquire masses we concentrate on the $4 \times 4$ submatrix. In the $\left(A, B, A^{*}, B^{*}\right)$ basis it is,

$$
M_{S}^{2}=\frac{\partial^{2} V}{\partial \phi_{i} \partial \phi_{j}}=\left(\begin{array}{cccc}
m^{2}+4 \lambda^{2} x^{2} & 2 \lambda m x & -\lambda^{2} \mu^{2} & 0 \\
2 \lambda m x & m^{2} & 0 & 0 \\
-\lambda^{2} \mu^{2} & 0 & m^{2}+4 \lambda^{2} x^{2} & 2 \lambda m x \\
0 & 0 & 2 \lambda m x & m^{2}
\end{array}\right)
$$

The scalar masses (really $m^{2}$ 's) are 0,0 , and $m^{2}+\frac{\lambda}{2}\left(4 \lambda x^{2} \pm \lambda \mu^{2} \pm \sqrt{16 m^{2} x^{2}+\lambda^{2}\left(\mu^{2}-4 x^{2}\right)}\right)$. We can immediately see another feature of spontaneous SUSY breaking in a renormalizable theory, there is a sum rule:

$$
\operatorname{Str} M^{2}=\sum(-1)^{2 J}(2 J+1) M_{J}^{2}=\sum_{\text {scalars }} M_{s}^{2}-2 \sum_{\text {fermions }} M_{F}^{2}=0
$$

This is true in all theories where SUSY is broken at the renormalizable level and immediately indicates a problem for coupling the MSSM to SUSY breaking directly - there would be superpartner lighter than its SM partner!

To see that this is true in general and not just a quirk of O'Raifeartaigh models recall that the scalar mass matrix is of the form,

$$
M_{S}^{2}: \frac{1}{2}\left(\Phi_{i}^{*} \Phi_{i}\right)\left(\begin{array}{cc}
\frac{\partial^{2} V}{\partial \phi_{i}^{*} \partial \phi_{j}} & \frac{\partial^{2} V}{\partial \phi_{i}^{*} \partial \phi_{j}^{*}} \\
\frac{\partial^{2} V}{\partial \phi_{i} \partial \phi_{j}} & \frac{\partial^{2} V}{\partial \phi_{i} \partial \phi_{j}^{*}}
\end{array}\right)\left(\begin{array}{c}
\Phi_{j} \\
\Phi_{j}^{*}
\end{array}\right)
$$


while the fermion mass matrix is,

$$
M_{F}: \frac{1}{2} \psi_{i} \frac{\partial W}{\partial \phi_{i} \partial \phi_{j}} \psi_{j}
$$

Since $V=\frac{\partial W}{\partial \phi_{i}} \frac{\partial W^{*}}{\partial \phi_{i}^{*}}$ we immediately see that $\operatorname{Tr} M_{S}^{2}=2 \operatorname{Tr} M_{F}^{2}$.

Question 10. Fayet-Iliopoulos terms. For a $U(1)$ gauge group there is one more gauge invariant operator that can be added to the Lagrangian, a Fayet-Iliopoulos term, $\int d^{4} \theta \kappa V$. Consider SUSY QED with an FI term and a vector like pair of "electrons", i.e.

$$
\left.\left(\Phi_{1}^{\dagger} e^{e V} \Phi_{1}+\Phi_{2}^{\dagger} e^{-e V} \Phi_{2}-\kappa^{2} V\right)\right|_{\theta^{4}}+\left.\left(\frac{1}{4} W_{\alpha} W^{\alpha}+m \Phi_{1} \Phi_{2}+\text { h.c. }\right)\right|_{\theta^{2}}
$$

Show that for the case $m^{2}>e \kappa^{2}$ SUSY is broken but the gauge symmetry is not but for $m^{2}<e \kappa^{2}$ both SUSY and the $U(1)$ are broken. Show that in both cases the supertrace is 0 , as expected.

\section{The MSSM}

Now we are in a position to discuss the supersymmetric version of the SM. There are many ways in which one can imagine embedding the SM within supersymmetry, the one which requires the introduction of the smallest number of superpartners is called the Minimal Supersymmetric Standard Model (MSSM). Before writing down its Lagrangian it is useful to first remind ourselves of the field content of the SM, written in terms of only LH fermions. The SM is based on the gauge structure $S U(3) \times S U(2) \times U(1)$ and under these groups it has 3 generations of matter fields that are in the following representations:

$$
\begin{aligned}
q_{i}=\left(u_{L}, d_{L}\right) & :\left(3,2, \frac{1}{6}\right) \\
u_{i}^{c} & :\left(\overline{3}, 1,-\frac{2}{3}\right) \\
d_{i}^{c} & :\left(\overline{3}, 1, \frac{1}{3}\right) \\
\ell=\left(v, e_{L}\right) & :\left(1,2,-\frac{1}{2}\right) \\
e_{i}^{c} & :(1,1,1)
\end{aligned}
$$

Gauge fields that are in the adjoint representation of the groups:

$$
\begin{aligned}
g & :(8,1,0) \\
A_{\mu}^{a} & :(1,3,0) \\
B_{\mu} & :(1,1,0)
\end{aligned}
$$

The last two mix after electroweak symmetry breaking. Finally there is the Higgs boson:

$$
h:\left(1,2, \frac{1}{2}\right)
$$




\begin{tabular}{c|c||c|c} 
SM Field & $S U(3), S U(2), U(1)$ & MSSM partner & Superfield \\
\hline \hline$q_{i}$ (LH quarks) & $\left(3,2, \frac{1}{6}\right)$ & $\tilde{q}_{i}$ (LH squarks) & $Q_{i}$ \\
$u_{i}^{c}(\mathrm{RH}$ top, charm, up) & $\left(\overline{3}, 1,-\frac{2}{3}\right)$ & $\tilde{u}_{i}^{c}(\mathrm{RH}$ stop, scharm, sup) & $U_{i}^{c}$ \\
$d_{i}^{c}$ (RH bottom, strange, down) & $\left(\overline{3}, 1, \frac{1}{3}\right)$ & $\tilde{d}_{i}^{c}(\mathrm{RH}$ sbottom, sstrange, sdown $)$ & $D_{i}^{c}$ \\
$\ell_{i}$ (LH leptons) & $\left(1,2,-\frac{1}{2}\right)$ & $\tilde{\ell}_{i}(\mathrm{LH}$ sleptons $)$ & $L_{i}$ \\
$e_{i}^{c}(\mathrm{RH}$ tau, muon, electron) & $(1,1,1)$ & $\tilde{e}_{i}^{c}(\mathrm{RH}$ stau, smuon, selectron) & $E_{i}^{c}$ \\
$h_{u}\left(h_{d}\right)$ (Higgs) & $\left(1,2, \frac{1}{2}\right) ;\left(1,2,-\frac{1}{2}\right)$ & $\tilde{h}_{u}\left(\tilde{h}_{d}\right)$ (higgsino) & $H_{u}\left(H_{d}\right)$
\end{tabular}

Table 3: Field content and naming conventions of the MSSM.

The simplest way to supersymmetrise is to place all the SM fields into superfields and introduce the necessary superpartners to fill out the superfields. For the fermions this requires introducing scalars (dubbed sfermions) and placing them in a chiral multiplets. We will denote the chiral superfield by the upper case version of the SM field, e.g. $q_{i} \rightarrow Q_{i}$. Superpartners of SM fields will be denoted with a tilde and the scalars have been given names by (unfortunately) adding an "s" to the front of the SM particles name, e.g. the superpartner of the electron (the selectron) is $\tilde{e}$.

The gauge bosons will require the introduction of femionic partners (dubbed gauginos) and will be placed in vector superfields. We will denote them as $V_{i}$ where $i=3,2,1$ denotes the size of the group. The fermionic partners take their name from the SM field and adding an "ino" on the end, e.g. the gluino, $\tilde{g}$, is the fermionic partner of the gluon, $g$.

So far in filling out the chiral superfields we have been introducing new bosonic partners. In the case of the Higgs however we are introducing a new chiral fermion and this leads to a problem. Chiral fermions contribute to anomalies and the introduction of one fermion charged under $S U(2) \times$ $U(1)$ will make the gauge symmetries anomalous. Also, the restriction in supersymmetry that the superpotential has to be a holomorphic function of the chiral superfields would forbid some of the necessary Yukawa couplings. Both of these facts can be avoided if we introduce not only a fermionic partner of the SM Higgs (by the naming convention called a Higgsino) but a second chiral superfield. Thus there are now two Higgs chiral superfields,

$$
\begin{aligned}
& H_{u}=\left(H_{u}^{+}, H_{u}^{0}\right):\left(1,2, \frac{1}{2}\right) \\
& H_{d}=\left(H_{d}^{0}, H_{d}^{-}\right):\left(1,2,-\frac{1}{2}\right)
\end{aligned}
$$

The total field content and the bizarre naming convention is collected in Table 3 .

With the field content in hand we may now procede to follow the mantra of effective field theory and write down all operators allowed by symmetry. Keeping only renormalizable operators we have Kähler terms of the form,

$$
K=Q^{\dagger} e^{V_{3}} Q+U^{c \dagger} e^{-V_{3}} U^{c}+D^{c \dagger} e^{-V_{3}} D^{c}+\ldots
$$

gauge kinetic terms of the form,

$$
\int d^{2} \theta \frac{1}{4 g_{3}^{2}} W_{\alpha}^{(3)} W^{(3) \alpha}+\ldots
$$




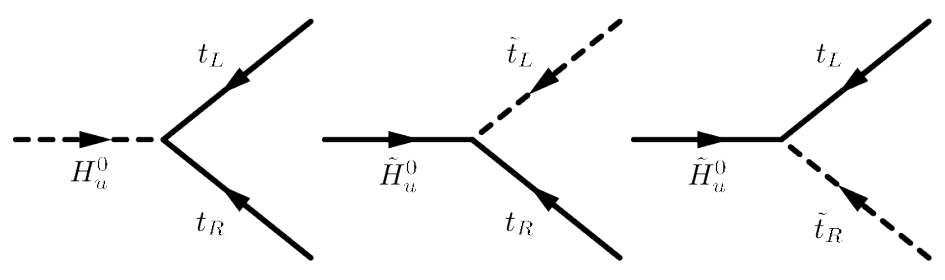

Figure 6: Top Yukawa couplings.

Finally, the superpotential which we discuss in two parts. First,

$$
W_{M S S M}=\mathbf{Y}_{\mathbf{U}} U^{c} Q H_{u}-\mathbf{Y}_{\mathbf{D}} D^{c} Q H_{d}-\mathbf{Y}_{\mathbf{E}} E^{c} L H_{d}+\mu H_{u} H_{d} .
$$

I have suppressed flavour and gauge indices for clarity. We can see again the need for the introduction of a second Higgs doublet, without it some of the SM fermions would be massless. As in the SM the fields may be rotated such that the Yukawas are diagonal, and since the third generation of SM fermions is appreciably heavier than first two the Yukawas are often approximated as $\mathbf{Y}_{\mathbf{U}} \approx \operatorname{diag}\left(0,0, y_{t}\right), \mathbf{Y}_{\mathbf{D}} \approx \operatorname{diag}\left(0,0, y_{b}\right)$ and $\mathbf{Y}_{\mathbf{E}} \approx \operatorname{diag}\left(0,0, y_{\tau}\right)$. The $\mu$-term is a mass term for the Higgsinos and will also, through F-terms, contribute to the scalar potential.

Expanding the superfields in $W_{M S S M}$ in their component fields gives us the Feynman rules for the SM particles and their superpartners. Concentrating on the top Yukawa term we can write down three different couplings all of size $y_{t}$ and we learn a very useful rule of thumb for understanding couplings in the MSSM, see Figure 6. Take any vertex in the SM and replace two of the particles with their superpartners and this is a vertex in the MSSM. This does not capture all the available couplings, for instance the F-term for $U^{c}$ leads to a four-point Higgs-squark coupling that has no SM counterpart, but does work for couplings involving at least one SM fermion coming from the superpotential and the gauge coupling terms.

Question 11. Put the flavour and gauge indices back into (4.7), paying close attention to $S U(2)$ indices which are contracted with $\varepsilon_{\alpha \beta}$, and confirm the signs.

In addition to these SM-like terms there are some other renormalisable operators allowed by the gauge symmetries that can be added to the superpotential,

$$
W_{\Delta B, L}=\kappa_{1}^{i j k} Q_{i} L_{j} D_{k}^{c}+\kappa_{2}^{i j k} L_{i} L_{j} E_{k}^{c}+\kappa_{3}^{i} L^{i} H_{u}+\kappa_{4}^{i j k} D_{i}^{c} D_{j}^{c} U_{k}^{c} .
$$

However, the first 3 of these operators violate lepton number, and the last is no better since it violates baryon number. Note that both $\kappa_{2}$ and $\kappa_{4}$ are antisymmetric under $i \leftrightarrow j$ because of the antisymmetry of the gauge indices, one is contracted with $\varepsilon^{\alpha \beta}$ and one with $f^{a b c}$. At the renormalizable level in the SM baryon and lepton symmetries are accidental, operators that would violate $B$ or $L$ are forbidden because of gauge symmetries; $B$ and $L$ are separately violated by non-perturbative processes, only $B-L$ is conserved. In the MSSM this accident no longer happens because superpartners allow us to construct the operators in (4.8). We could forbid these operators by fiat, as we will see the superpotential has an interesting non-renormalisation property so that even if there is no symmetry forbidding these operators once their coefficients are set to zero they won't be generated in perturbation theory, but this is not appealing. 
These operators could be forbidden if we introduced a new symmetry, the price we have to pay for wanting to solve the hierarchy problem. For instance we could introduce an R-symmetry as in Section 3.7 where $R\left[Q, U^{c}, D^{c}, L, E^{c}\right]=1 / 2$ and $R\left[H_{u}, H_{d}\right]=1$. This would forbid the $W_{\Delta B, L}$ terms while allowing the $W_{M S S M}$ terms. However, as we will soon see, this is too restrictive and would forbid mass terms for gauginos. Instead we consider a discrete $Z_{2}$ subgroup of the $U(1)$ R-symmetry under which superpartners flip sign and SM fields do not. Under this R-parity ${ }^{10}$ the fields have charge,

$$
P_{R}=(-1)^{3(B-L)+F}
$$

Under the parity SM fields are even and superpartners are odd and it has several interesting implications:

\section{Superpartners and SM particles cannot mix}

2. The lightest parity odd particle (LPOP) is a superpartner and the lightest supersymmetric particle (LSP) is stable. It turns out that the LSP is often a neutral state and has exactly the right properties to be the DM!

3. Superpartners must be made in pairs, and when they decay they eventually decay down to an odd number of LSPs. If this decay is prompt (and the LSP is neutral) they leave a missing energy signature in detectors.

This idea of parity oddness for new particles is so successful that it has been borrowed many times for other BSM scenarios e.g. KK-parity leading to LKPs of extra dimensions[38], T-parity and LTPs of Little Higgs scenarios[39]. In the rest of these lectures we will assume that R-parity is an exact symmetry of the MSSM but it is also possible that it is broken, that there is another symmetry that protects protons from decay or that the $\kappa$ are tuned to be small [40, 41]. If this were the case then SUSY would lose its dark matter candidate and depending on the timescale for decay its missing energy signature in colliders. To see how small the couplings to the light quarks would have to be consider the case of $\kappa_{1}$ and $\kappa_{4}$ non-zero, then there would be a tree-level diagram, involving squark exchange, that would lead to proton decay. Although an exact calculation is complicated, we need to know the details of the quark make-up of the proton, we can estimate the proton lifetime,

$$
\tau^{-1}=\Gamma \sim \frac{\left|\kappa_{1} \kappa_{4}\right|^{2}}{16 \pi} \frac{m_{p}^{5}}{m_{\tilde{q}}^{4}} \Rightarrow \tau \approx\left|\kappa_{1} \kappa_{4}\right|^{-2}\left(\frac{m_{\tilde{q}}}{1 \mathrm{TeV}}\right)^{4} \times 10^{-11} s .
$$

The proton lifetime is at least $\sim 10^{32}$ years implying that the relevant $\kappa$ have to be very small, $|\kappa| \lesssim 10^{-12}$.

Now that we have forbidden the bad renormalisable operators ${ }^{11}$ we have a fully supersymmetric version of the SM. A parameter count shows that the number of parameters is one smaller than that in the SM since the Higgs potential is entirely determined by the D-terms. However, this is not a fully realistic model since we know that the superpartners are not degenerate with their SM

\footnotetext{
${ }^{10}$ Equivalently another possibility is matter parity where parity is assigned by $P_{M}=(-1)^{3(B-L)}$.

${ }^{11}$ There are higher dimension operators, such as $Q Q Q L$, that can contribute in loops to proton decay. Depending on the scale that suppresses these operators they too can be a concern in supersymmetric theories [42].
} 
cousins. To break this degeneracy requires us to break SUSY and will introduce a multitude (105!) of new parameters [43]. SUSY is great, breaking it is where the trouble begins.

\subsection{SUSY breaking in the MSSM}

The supertrace condition (3.65) on tree-level SUSY breaking predicts superpartners lighter than the heaviest SM particle in each charge sector of the SM, i.e. sleptons are lighter than the tau, squarks are lighter than the top etc. This is clearly ruled out, which leads to the typical scenario for introducing SUSY breaking into the MSSM. We introduce some hidden sector whose dynamics is such that the vacuum of this sector is not supersymmetric [44] but is sufficiently heavy that the supertrace condition is not a concern. There are then some "messenger" fields which couple the MSSM to the dynamical SUSY breaking sector. The SUSY breaking in this dynamical SUSY breaking sector is then mediated to the MSSM through the messengers. For instance in gauge mediation [45] the messengers have SM gauge quantum numbers, whereas in gravity mediation the messenger fields are unspecified fields whose mass is at the Planck scale. Integrating out the messenger fields results in couplings between the SUSY breaking and the MSSM, the size of these couplings depends on the details of the mediation mechanism - a subject worthy of a series of TASI lectures itself [25]. However, the list of SUSY breaking operators is finite and one can parametrise all possibly combinations by considering just these operators, which we do below.

\subsection{Spurions}

For the purpose of these lectures, and much of SUSY phenomenology, it is sufficient to carry out a "spurion" analysis. Spurion analyses are a useful tool when one wishes to keep track of how a symmetry is broken, any parameter that breaks a symmetry can be elevated to the status of a field and the symmetry restored by assigning the appropriate transformation properties to the field. The field is not dynamical, its sole purpose is to get a vacuum expectation value which breaks the symmetry, restoring the parameter to the Lagrangian, but in so doing it helps us keep track of allowable operators. We have seen this before in the SM, at energies below the $W$ mass we write down the QCD Lagrangian including mass terms for the quarks. But in reality, once we learn about $S U(2)_{W}$, we realise that these quark masses break $S U(2)_{W}$ which can be restored if the mass is thought of as transforming under the $S U(2)_{W}$ symmetry. In this case the spurion is nothing more than the SM Higgs. In SUSY there need not be a physical particle associated with the spurion, or it may be too heavy to ever be accessible, but the restoration of SUSY will still be a useful tool.

In SUSY the available spurions whose VEV break the symmetry without also breaking Lorentz invariance are the F-term of a chiral superfield, $X=\theta^{2} F$, or the D-term of a $U(1)$ vector superfield, $W_{\alpha}^{\prime}=\theta_{\alpha} D$. With these in hand we can ask what are the leading operators involving these spurions that will lead to SUSY breaking terms in the Lagrangian. In the MSSM the only relevant spurion is $X$ and the important operators, generated at the messenger scale $(M)$, are:

\section{Scalar mass}

$$
c^{i j} \int d^{4} \theta \frac{X^{\dagger} X}{M^{2}} Q_{i}^{\dagger} Q_{j},
$$

which leads to a scalar mass ${ }^{2}$ term in the Lagrangian of

$$
-\left(m^{2}\right)^{i j} \tilde{q}_{i}^{*} \tilde{q}_{j},
$$


with $\left(m^{2}\right)^{i j}=-c^{i j}\left(F_{X} / M\right)^{2}$. This operator exists whether $X$ is a MSSM singlet or not. The $c^{i j}$ can have new flavour structure and if the sfermions are not well above the weak scale this can potentially lead to visible flavour violating effects. Certain mediation mechanisms, for instance gauge mediation, predict that $c^{i j} \propto \delta^{i j}$ which avoids this problem. In gravity mediation there is no such prediction but nonetheless it is often assumed that the scalar masses generated at the Planck scale are flavour diagonal, primarily to avoid these strong constraints.

\section{Gaugino mass}

$$
\frac{1}{2} c_{i} \int d^{2} \theta \frac{X}{M} W^{\alpha} W_{\alpha}
$$

which leads to a Majorana gaugino mass term in the Lagrangian of

$$
-\frac{1}{2} m_{i} \tilde{\lambda}^{\alpha} \tilde{\lambda}_{\alpha}
$$

where $i$ here runs over the three gauge groups of the MSSM, and $m_{i}=-c_{i} F / M$. This operator can only be written down if $X$ is a MSSM singlet. If this is not the case one would expect the scalar masses (4.11) to be far larger than the gaugino masses (4.13).

\section{A term}

$$
\int d^{2} \theta \frac{X}{M}\left(A_{u}^{i j} U_{i}^{c} Q_{j} H_{u}-A_{d}^{i j} D_{i}^{c} Q_{j} H_{d}-A_{e}^{i j} E_{i}^{c} L_{j} H_{d}\right)
$$

which leads to scalar trilinear terms in the Lagrangian of

$$
a_{u}^{i j} \tilde{u}_{i}^{c} \tilde{q}_{j} h_{u}-a_{d}^{i j} \tilde{d}_{i}^{c} \tilde{q}_{j} h_{d}-a_{e}^{i j} \tilde{e}_{i}^{c} \tilde{\ell}_{j} h_{d}
$$

with $a^{i j}=A^{i j} F / M$. As for the gaugino masses this operator requires that the spurion is a MSSM singlet. Furthermore, the A-terms are another new source of flavour violation and so have strong constraints on the sizes of the flavour off-diagonal terms.

\section{$b$ term}

$$
B \int d^{4} \theta \frac{X^{\dagger} X}{M^{2}} H_{u} H_{d},
$$

which leads to a scalar mass ${ }^{2}$ term in the Lagrangian of

$$
-b h_{u} h_{d}
$$

with $b=-B(F / M)^{2}$. If $X$ is a singlet then a $\mu$ term, a supersymmetric parameter, can also be generated from $X^{\dagger} H_{u} H_{d} / M$ in the Kähler potential. For successful electroweak symmetry breaking the supersymmetric mass parameter, $\mu$, must be around the weak scale and the SUSY preserving and breaking parameters related by $b \sim \mu^{2}$. If both these two operators are generated with comparable coefficients, as can occur, for example, in gravity mediated theories [46], then this provides a solution to the $\mu-b$ problem.

These are the leading operators discussed in the context of the MSSM. There are higher dimension operators that are typically generated with small coefficients at the messenger scale. In the absence of MSSM gauge singlets these additional operators have also been shown [47] to be 
"soft" and $X^{\dagger} X Q H_{u}^{\dagger} D^{c} / M^{3}$. Although they are typically small at the messenger scale these operators may be generated through renormalisation group running and can lead to interesting "wrong-type" Higgs couplings.

If the field content of the MSSM is extended then there are more operators that can be written down. One interesting possibility, that uses a D-term spurion, is that of supersoft SUSY breaking[48]. The MSSM is extended by adding chiral superfields, $A^{i}$, that transform in the adjoint representation of $U(1), S U(2)$ and $S U(3)$, for $i=1,2,3$, respectively. This now allows us to write down Dirac gaugino mass terms:

\section{Supersoft term}

$$
\sqrt{2} \int d^{2} \theta \frac{W^{\prime \alpha}}{M} W_{\alpha}^{i} A^{i}
$$

which results in a gaugino-adjoint Dirac mass, a mass term for (the real part of) the scalar adjoint, and a scalar tri-linear term,

$$
-m_{D} \tilde{\lambda}_{i} \tilde{a}_{i}-m_{D}^{2}\left(a_{i}+a_{i}^{*}\right)^{2}-\sqrt{2} m_{D}\left(a_{i}+a_{i}^{*}\right)\left(\sum_{j} g_{k} q_{j}^{*} t_{a} q_{j}\right),
$$

where $m_{D}=D^{\prime} / M$ and $q$ represents all MSSM fields charged under gauge group $i$. Models with just a D-term spurion have interesting renormalization properties $[49,50,51,52,53$, 48, 54].

\subsection{Superpartner mass spectra}

We now turn to the masses of the superpartners. We will focus on those fields most connected to DM, but will include a brief discussion of non-DM candidates for completeness. The requirement that DM be electrically neutral leads to three different DM candidates within SUSY: sneutrinos $(\tilde{v})$; an admixture of the higgsinos, wino, and bino (a so-called neutralino); and the gravitino.

Sneutrinos are the scalar superpartner of the SM neutrinos. Since they carry electroweak charge they have a coupling to the $Z$ boson and a large scattering rate off nuclei. They are ruled out by direct detection searches. However, if the mass eigenstates are split in some way, perhaps through mixing with a singlet or a lepton-number violating mass term, so the $Z$-coupling is off diagonal, then they can be an inelastic DM candidate [55].

Neutralinos are the mass eigenstates made from the higgsinos, winos and bino, they are Majorana fermions. They are the most common DM candidate in SUSY and their properties depend on how these states mix to form the mass eigenstates. After electroweak symmetry breaking the neutral components of the Higgsinos, $\tilde{h}_{u}$ and $\tilde{h}_{d}$, will mix with the bino, $\tilde{B}$, the partner of the $U(1)_{Y}$ gauge boson and the wino, $\tilde{W}^{0}$, the partner of the neutral component of the $S U(2)_{W}$ gauge boson. The mass eigenstates are called neutralinos and are variously denoted as $\tilde{N}_{i}$,

\footnotetext{
${ }^{12}$ They don't generate quadratic divergences, only logarithmic ones.
} 
$\tilde{\chi}_{i}^{0}, \tilde{Z}_{i}$, but in all cases $i$ labels the mass eigenstates from lightest, $i=1$ to heaviest $i=4$. Extensions of the MSSM will naturally have more neutralinos.

We define $\Psi_{0}^{T}=\left(\tilde{B}, \tilde{W}, \tilde{h}_{d}, \tilde{h}_{u}\right)$ which has a (Majorana) mass term in the Lagrangian of $-\frac{1}{2} \Psi_{0}^{T} M_{N} \Psi_{0}+$ c.c. with

$$
M_{N}=\left(\begin{array}{cccc}
m_{1} & 0 & -c_{\beta} s_{W} M_{Z} & s_{\beta} s_{W} M_{Z} \\
0 & m_{2} & c_{\beta} c_{W} M_{Z} & -s_{\beta} c_{W} M_{Z} \\
-c_{\beta} s_{W} M_{Z} & c_{\beta} c_{W} M_{Z} & 0 & -\mu \\
s_{\beta} s_{W} M_{Z} & -s_{\beta} c_{W} M_{Z} & -\mu & 0
\end{array}\right) .
$$

The real eigenvalues of this complex symmetric matrix can be found by diagonalising, $M_{N}^{\text {diag }}=U^{*} M_{N} U^{\dagger}$. If the off diagonal terms due to electroweak symmetry breaking are small relative to the other entries in the matrix then the mixing is small and the lightest neutralino will be mostly bino-, Higgsino- or Wino-like. In all cases the lightest neutralino is the DM and is a Majorana fermion.

Consider the annihilation of $\chi$ to a SM fermion/anti-fermion final state, $\chi \chi \rightarrow f \bar{f}$. Light fermions in the SM have definite helicity and since they are made back-to-back in the centre of mass frame the final state must have $S=J=1$. The initial state must be odd under particle exchange with the two lowest modes being $L=0, S=0 \Rightarrow J=0$ and $L=1, S=$ $1 \Rightarrow J=0,1,2$. Thus the only way to have overlap between the initial and final state is if the neutralinos annihilate in the $\mathrm{P}$-wave, $\langle\sigma v\rangle \propto v^{2}$, or if there is a fermion mass insertion in the final state.

Question 12. By writing down examples of Feynman diagrams that lead to neutralino annihilation confirm that S-wave processes are all proportional to final-state fermion mass.

Rather than analyse in gory detail the prospects for the neutralino sector to be the origin of thermal dark matter ${ }^{13}$, it is instructive to consider the case of the lightest neutralino being a pure state. If the lightest neutralino is pure Bino then the dominant annihilation is into righthanded leptons, since they have the largest hypercharge. This process proceeds through $t$ channel exchange of sleptons with $\langle\sigma v\rangle \sim g_{Y}^{4} M_{1} T /\left(2 \pi m_{\tilde{e}}^{4}\right)$, notice the factor of $v^{2} \sim T / m$ as predicted by earlier arguments. To get the correct relic abundance the sleptons must be light (but heavier than the bino), which is in tension with results from LEP requiring $m_{\tilde{e}} \gtrsim 100$ $\mathrm{GeV}$. As the bino and sleptons become heavier the annihilation cross section drops and there is too much DM.

For Higgsino DM the largest annihilation modes are into $W$ and $Z$ gauge bosons (there is an annihilation to heavy fermions too) and so the cross sections are considerably larger than for bino, resulting in typically too little dark matter. The higgsino sits inside an $S U(2)$ doublet so comes with a partner state which is charged, whose mass is split from the neutral state only through loop effects. At one loop, for states heavier than the $W, Z$, the one-loop splitting between a neutral state and a state of charge $Q$ in a multiplet with hypercharge $Y$ is given

\footnotetext{
${ }^{13}$ It has been done elsewhere [56].
} 
[57] by,

$$
\Delta M \approx Q\left(Q+\frac{2 Y}{\cos \theta_{W}}\right) \alpha_{2} M_{W} \sin ^{2} \frac{\theta_{W}}{2} .
$$

For a higgsino this splitting is $\sim 350 \mathrm{MeV}$ and so at the freeze out temperature $T \sim m / 20$ these states will also be plentiful in the thermal bath. The relic abundance is sensitive to the presence of these states since they can annihilate with the DM, in a process called coannihilation which will be discussed in detail below, see Section 5.1. The approximate annihilation cross section for higgsinos is $\langle\sigma v\rangle \approx g_{2}^{4} /\left(512 \pi \mu^{2}\right)$ and the correct relic abundance occurs for $\mu \sim 1.1 \mathrm{TeV}$.

So far I have discussed one light neutral state nearly degenerate with one charged state, but there are actually two neutral states coming from the higgsino sector. In the absence of all other masses these two neutral states, $\tilde{h}_{u}^{0}, \tilde{h}_{d}^{0}$, would make a Dirac fermion. Such a pure higgsino would be ruled out as DM since it would have a large coupling to the $Z$ boson and should have been seen in direct detection. However, electroweak symmetry breaking mixes the higgsinos with the bino and wino and splits the two neutral states. This splitting [58] is,

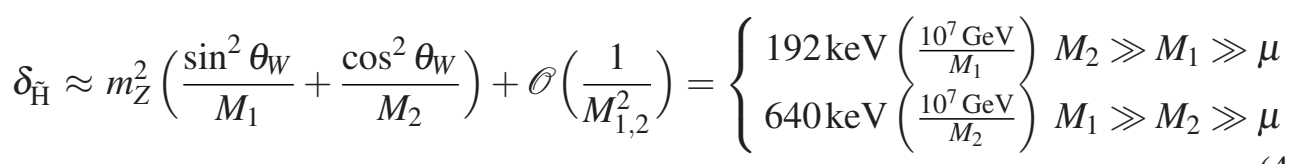

Thus, unless the other neutralinos are very heavy there is a sizeable splitting between the two neutral states. Interestingly, with very heavy bino and wino the splitting is comparable to the kinetic energy of the incoming WIMP and the kinematics is altered. This is an example of a scenario called inelastic dark matter [59].

The case of Wino DM is similar to the higgsino but with an even smaller charged-neutral splitting $\delta_{\tilde{\mathrm{W}}} \approx 166 \mathrm{MeV}$ and a larger annihilation cross section $\langle\sigma v\rangle \approx 3 g_{2}^{4} /\left(16 \pi M_{2}^{2}\right)$. The correct reclic abundance occurs for wino mass of $\sim 3 \mathrm{TeV}$. One important difference between the wino and higgsino is that the wino does not have a tree level coupling to the nucleus, so can evade direct detection bounds. There is a loop induced spin-independent scattering cross section $[60,61]$ but it is small.

Gravitino In exactly the same way that a spontaneously broken bosonic global symmetry gives rise to a massless goldstone boson, spontaneously broken SUSY results in a massless goldstone fermion, the goldstino - recall Section 3.8. As discussed earlier, SUSY is a spacetime symmetry and once gravity is included SUSY must become a local symmetry. Gauged SUSY is commonly called supergravity, or SUGRA. In direct analogy to the eating of the goldstone boson by the gauge boson associated with the gauged bosonic symmetry (thus giving the gauge boson a mass) the goldstino is eaten in SUGRA. In this case the particle doing the eating is the fermionic partner of the spin-2 graviton: the spin-3/2 gravitino.

For concreteness we will consider the SUSY breaking to come from a superfield $X$ acquiring an $F$-term vev, $X \sim F \theta^{2}$. In this case the mass of the gravitino is given by $m_{3 / 2} \sim F / M_{\mathrm{Pl}}$. This should be compared to the masses of, for example, the gauginos $m_{1 / 2} \sim F / M_{\text {mess }}$, with $M_{\text {mess }}$ the scale of the messengers that communicate SUSY breaking to the SM fields. The 
couplings of the goldstino scale as $1 / F$. For low-scale SUSY breaking where the messenger scale is far below the Planck scale the gravitino will be the lightest parity odd particle, and will be (at least part of) DM.

Gravitino DM can be produced through two mechanisms: through decays of the next-to lightest SUSY particle (NLSP), a non-thermal production mechanism, or thermal production after inflation. The first mechanism is sometimes called the "superWIMP" scenario [62]. The lifetime for the decay of the NLSP into the gravitino is $\tau_{\mathrm{NLSP} \rightarrow \tilde{G}} \sim\left(m_{\mathrm{NLSP}}^{5} / F^{2}\right)^{-1}$. If this is longer than the timescale for NSLP freeze out we can approximately determine the abundance of the gravitino by noting that every NLSP will decay into an LSP. Thus, $\Omega_{3 / 2}=\Omega_{1 / 2} \frac{m_{3 / 2}}{m_{1 / 2}}$. If this decay happens late the energetic SM states produced in the decay can cause trouble, for instance they may dissociate primordial elements produced in BBN [63].

The second production mechanism occurs when two particles in the thermal bath collide and produce a gravitino. The gravitino production cross section is small $\sigma \sim g^{2} m_{1 / 2}^{2} / F^{2}$ and the production rate is slow. The initial state of the universe has no gravitinos present (assuming the inflaton does not directly decay into gravitinos) and the production rate is so slow that they never get into thermal equilibrium. Thus, the annihilation processes can be ignored compared to those of production and the Boltzmann equation that governs the evolution is,

$$
\dot{n}_{3 / 2}+3 H n_{3 / 2}=\langle\sigma v\rangle n_{\mathrm{rad}}^{2},
$$

where the inverse process of gravitino annihilation (the term $-\langle\sigma v\rangle n_{3 / 2}^{2}$ ) is ignored since the number density of gravitinos is small. Since $n_{\mathrm{rad}} \sim T^{3}$ and $H \sim T^{2} / M_{\mathrm{Pl}}$ we can solve (4.24) to find the abundance of gravitinos,

$$
Y_{3 / 2} \sim \frac{g^{2} m_{1 / 2}^{2} T_{R}}{m_{3 / 2}^{2} M_{\mathrm{Pl}}}
$$

where $T_{R}$ is the reheat temperature and is the highest temperature reached after the inflaton decays. The abundance of gravitinos is dominated by its production at high temperatures and inserting $Y_{3 / 2}$ into (2.15) we see this places an upper bound on the reheat temperature.

Having described the DM candidates in SUSY we now very briefly discuss the other superpartner masses. The charged components of the fields that make up the neutralinos also mix. Define $\Psi_{ \pm}^{T}=\left(\tilde{W}^{+}, \tilde{h}_{u}^{+}, \tilde{W}^{-}, \tilde{h}_{d}^{-}\right)$which has a Lagrangian term $-\frac{1}{2} \Psi_{ \pm}^{T} M_{C} \Psi_{ \pm}$with

$$
M_{C}=\left(\begin{array}{cccc}
0 & 0 & m_{2} & \sqrt{2} s_{\beta} M_{W} \\
0 & 0 & \sqrt{2} c_{\beta} M_{W} & \mu \\
m_{2} & \sqrt{2} c_{\beta} M_{W} & 0 & 0 \\
\sqrt{2} s_{\beta} M_{W} & \mu & 0 & 0
\end{array}\right) .
$$

It is easier to work with the non-trivial $2 \times 2$ block of $M_{C}, M$. Since $M$ is not symmetric it is diagonalised by two different unitary transformations, $M^{\text {diag }}=L^{*} M R^{\dagger}$. The masses of the charginos, 
denoted by $\tilde{C}_{i}, \tilde{\chi}_{i}^{ \pm}$or $\tilde{W}_{i}^{ \pm}$, are given by

$$
\begin{aligned}
M_{C_{1}, C_{2}}= & \frac{1}{2}\left[\left|m_{2}\right|^{2}+|\mu|^{2}+2 M_{W}^{2}\right. \\
& \left.\mp \sqrt{\left(\left|m_{2}\right|^{2}+|\mu|^{2}+2 M_{W}^{2}\right)^{2}-4\left|\mu m_{2}-M_{W} s_{2 \beta}\right|^{2}}\right] .
\end{aligned}
$$

Given the issues with generating the correct size for the $\mu$-term, discussed earlier, one might wonder if these problems are removed if the $\mu$-term is somehow forbidden. However, notice that $\mu=0$ would lead to both a massless neutralino and a chargino below the $W$ mass, which is ruled out by LEP searches. However, extensions of the MSSM can be built that do not have a $\mu$-term [64], or where the $\mu$-term is generated from SUSY breaking. The later case is a commonly discussed extension of the standard model: the exension of the MSSM by a gauge singlet chiral superfield, $S$, called the next-to-minimal supersymmetric standard model (NMSSM).

Question 13. By finding the eigenvalues of $M^{\dagger} M$ confirm (4.27).

The sfermion masses receive contributions from various sources. I will discuss the case of the stops, the other squarks and sleptons masses follow in analogous fashion, and I will also ignore potential flavour violating contributions to the mass matrices. Working in the basis $\Psi_{t}^{T}=\left(\tilde{q}_{3}, \tilde{u}_{3}^{c}\right)$ the stop mass matrix is

$$
M_{t}=\left(\begin{array}{cc}
M_{t}^{2}+m_{\tilde{q}_{3}}^{2}+\Delta_{\tilde{q}_{3}} & M_{t}\left(A_{t}^{*}-\mu \cot \beta\right) \\
M_{t}\left(A_{t}-\mu \cot \beta\right) & M_{t}^{2}+m_{\tilde{u}_{3}^{c}}+\Delta_{\tilde{u}_{3}^{c}}
\end{array}\right) .
$$

The soft scalar masses $m_{\tilde{q}_{3}}^{2}$ and $m_{\tilde{u}_{3}^{c}}$ arise from SUSY breaking as described in (4.11). The $\Delta_{\tilde{f}}$ terms arise from the $S U(2)_{W}$ and $U(1)_{Y}$ D-terms in the scalar potential. For example the relevant piece of the $U(1)_{Y}$ D-term is $-\frac{g^{\prime}}{2}\left(\left|h_{u}^{0}\right|^{2}-\left|h_{d}^{0}\right|^{2}+\sum_{i} \tilde{f}_{i}^{*} Y_{i} \tilde{f}_{i}\right)$. In general for a sfermion, $\tilde{f}$, the D-term contributions to the mass matrix are

$$
\Delta_{\tilde{f}}=\left(T_{3}-Q \sin ^{2} \theta_{W}\right)_{\tilde{f}} \cos 2 \beta M_{Z}^{2} .
$$

For third generation sfermions like the top there are F-term contributions, from the F-terms for $U_{3}, Q_{3}$ and $H_{U}$, these give the contributions proportional to $\mu$ in (4.28). Finally there are $A$ term contributions [see (4.16)] where in (4.28) I have followed the oft-used convention of $a_{i}=$ $y_{i} A_{i}$. Similar matrices exist for the other squark and sleptons, however for down-type squarks and sleptons the down-type Higgs F-terms is involved, thus one must also make the replacement $\tan \beta \leftrightarrow \cot \beta$. The mass matrix (4.28) must be diagonlised and the resulting mass eigenstates are denoted $\tilde{t}_{1}$ and $\tilde{t}_{2}$ with the convention that $m_{\tilde{t}_{1}}^{2}<m_{\tilde{t}_{2}}^{2}$.

The only remaining superpartner left to discuss is the gluino. Since it is the only octet of colour it has nothing to mix with and its mass is simply given by $M_{3}$.

\section{Supersymmetric Dark Matter}

As mentioned in Section 4, the existence of relevant baryon- and lepton-number violating operators in the MSSM superpotential necessitates the introduction of R-parity ${ }^{14}$. This approach has

\footnotetext{
${ }^{14}$ Actually, there are alternative approaches $[40,41]$ that allow R-parity to be broken without dangerous rates of proton decay, leading to an unstable LSP, I will not discuss them further here.
} 
the happy byproduct of making the lightest superpartner absolutely stable, and in a large fraction of parameter space the LSP has the correct properties to be the cosmological DM. We discussed the possible candidates, along with the rest of the SUSY spectrum in Section 4.3. We now turn to details of DM phenomenology within the MSSM, although many of the features we will discuss show up in other WIMP models. We start by discussing freeze out in the MSSM, before turning to ways to detect WIMP DM - directly, indirectly and at colliders. The definitive work on this topic [65] is now somewhat dated but still a great reference.

\subsection{Freeze out revisited}

In Section 2 we discussed the freeze out of a single DM particle coupled to the thermal bath. However, in the MSSM, and other models of BSM physics, it may not be sufficient to consider just the evolution of the DM particle in isolation. The particle spectrum of SUSY is rich and the DM may have companion states present in the bath, or couplings to other R-parity odd particles, that dramatically alter its relic abundance. For instance, the neutralinos can have nearly degenerate chargino partners, or squarks and sleptons maybe nearby in mass.

There are situations, for historical reasons dubbed "three exceptional cases" [66], that occur in regions of parameter space that require more detailed analysis:

Coannihilation If there is another MSSM state with mass within a few percent of the DM mass then its abundance at freeze out will not be negligible. For larger mass splittings the MaxwellBoltzmann suppression of the heavier state's thermal abundance is large and it can be ignored. The additional light state(s) can take part in annihilation and other processes that determine the relic abundance. Such a situation is referred to as "coannihilation" and can occur in the MSSM [67], for example where the DM bino coannihilates with a nearly degenerate stau.

Consider the general situation with $N$ SUSY states whose abundances are non-negligible at the time of freeze out, we label them as $\chi_{1 \ldots N}$ with $\chi_{1}$ the lightest and ultimately all of the DM. There are several classes of processes that can have impact on the final relic abundance: co-annihilation of $\chi_{i} \chi_{j}$,

$$
\sigma_{i j}=\sum_{X} \sigma\left(\chi_{i} \chi_{j} \rightarrow X\right)
$$

conversion through scattering,

$$
\sigma_{X i j}^{\prime}=\sum_{Y} \sigma\left(\chi_{i} X \rightarrow \chi_{j} Y\right)
$$

and conversion through decay,

$$
\Gamma_{i j}=\sum_{X} \Gamma\left(\chi_{i} \rightarrow \chi_{j} X\right)
$$

We denote SM states by $X, Y$. The simple Boltzmann equation of (2.12) now becomes an 
intimidating system of coupled equations for each species,

$$
\begin{aligned}
\frac{d n_{i}}{d t}+3 H n_{i}= & -\sum_{j=1}^{N}\left\langle\sigma_{i j} v_{i j}\right\rangle\left(n_{i} n_{j}-n_{i}^{\mathrm{eq}} n_{j}^{\mathrm{eq}}\right) \\
& -\sum_{j \neq i}\left(\left\langle\sigma_{X i j}^{\prime} v_{i j}\right\rangle\left(n_{i} n_{X}-n_{i}^{\mathrm{eq}} n_{X}^{\mathrm{eq}}\right)-\left\langle\sigma_{X j i}^{\prime} v_{j i}\right\rangle\left(n_{j} n_{X}-n_{j}^{\mathrm{eq}} n_{X}^{\mathrm{eq}}\right)\right) \\
& -\sum_{j \neq i}\left(\Gamma_{i j}\left(n_{i}-n_{i}^{\mathrm{eq}}\right)-\Gamma_{j i}\left(n_{j}-n_{j}^{\mathrm{eq}}\right)\right) .
\end{aligned}
$$

Note, however, that any of the heavier states, $\chi_{2 \ldots N}$ will decay down to the LSP so the total WIMP abundance is $\sum_{i} n_{i} \equiv n$. If we sum up all the equations we see that the second and third lines in (5.4) sum to zero. Furthermore, the $\chi_{i}$ are kept in thermal equilibrium through their scattering with SM states. Typically $\sigma_{X i j}^{\prime}$ is comparable to $\sigma_{i j}$ but $n_{X} \gg n_{i}$, since the SM follows a relativistic distribution rather than the non-relativistic distribution of $\chi_{i}$. This means that the $n_{i}$ are thermal and $n_{i} / n=n_{i}^{\mathrm{eq}} / n^{\mathrm{eq}}$. This then allows us to write a Boltzmann equation for $n=\sum_{i} n_{i}$ that is very similar to the single species case (2.12),

$$
\dot{n}+3 H n=\left\langle\sigma_{\mathrm{eff}} v\right\rangle\left(n_{\mathrm{eq}}^{2}-n^{2}\right), \quad\left\langle\sigma_{\mathrm{eff}} v\right\rangle=\sum_{i, j}\left\langle\sigma_{i j} v_{i j}\right\rangle \frac{n_{i}^{\mathrm{eq}}}{n^{\mathrm{eq}}} \frac{n_{j}^{\mathrm{eq}}}{n^{\mathrm{eq}}}
$$

It is clear from (5.5) that states with mass near the WIMP mass, and therefore appreciable thermal abundance, can alter the effective annihilation cross section. As an example, for the pure bino/Higgsino/wino cases discussed in Section 4.3 coannihilation can be important. For the bino there is possible coannihilation with the right-handed sleptons, for the pure Higgsino or wino the charged and neutral states are nearly degenerate and taking into account coannihilation is critical.

$s$-channel pole The annihilation cross section can be altered if there is a state whose mass is close to twice the DM mass. The DM may annihilate through an $s$-channel resonance as can occur, for instance, in the MSSM with a neutralino that is an admixture of gaugino and higgsino annihilating through the $A^{0}$ pole; the so-called " $A$ /higgs funnel". Consider a WIMP annihilating through an $s$-channel mediator of mass $M$ and width $\Gamma$, with annihilation cross section

$$
\sigma v=\frac{\alpha s}{\left(s-M^{2}\right)^{2}+M^{2} \Gamma^{2}}
$$

The freeze out of DM takes place with $x \sim 20$, so the speed of DM is $\beta \sim 1 / 3$ and $s \sim 4.4 \mathrm{~m}^{2}$, as opposed to the present day annihilation where $v \sim 0$ and $s \sim 4 m^{2}$. For mediator mass around this centre of mass energy, $M \sim 4 m^{2}$, either present day or freeze-out annihilation can be enhanced by $\sim m^{2} / \Gamma^{2}$. Furthermore, when calculating thermally averaged cross sections one must take care in integrating over the Boltzmann distribution since the cross section is highly peaked for velocities that give $s \sim M^{2}$.

Forbidden channels The final of the three exceptions [66] is annihilation into "forbidden channels". These are annihilations of DM into a final state that at zero velocity is not allowed. If the channel $\chi \chi \rightarrow X Y$ is allowed by symmetries but $m_{X}+m_{Y}>2 m_{\chi}$ then it cannot happen 
with DM at rest. However, for freeze out the annihilations occur between WIMPs that have a Maxwell-Boltzmann distribution, so there will always be particles in the tails of the distribution with sufficient energy for this process to occur. If the mass splitting is not too large and this process has a large cross section then the Boltzmann suppression may be overcome and this "forbidden" channel can affect the relic abundance.

Solving for the DM abundance in the MSSM is a complicated business due to the complexity of the spectrum and couplings, and the number of diagrams that contribute to the annihilation. Thankfully computer codes such as DarkSUSY [68] and MicrOMEGAs [69] exist that can numerically solve (5.4) taking into account these three exceptions.

Question 14. Co-annihilation a. If co-annihilation modes are available during freeze out they can dramatically alter the final DM abundance. As shown in the text, including the additional channels leads to an "effective" annihilation cross section,

$$
\left\langle\sigma_{e f f} v\right\rangle=\sum_{i j}\left\langle\sigma_{i j} v_{i j}\right\rangle \frac{n_{i}^{e q}}{n^{e q}} \frac{n_{j}^{e q}}{n^{e q}},
$$

with $n=\sum_{i} n_{i}$. Consider the toy example of two states in the dark sector $\chi_{1,2}$ where $m_{1}<m_{2} \cdot \chi_{1}$ has only weak interactions whereas $\chi_{2}$ has strong interactions. We thus take the various annihilation cross sections to be in the ratios $\left\langle\sigma_{22} v\right\rangle=\frac{\alpha_{s}}{\alpha}\left\langle\sigma_{21} v\right\rangle=\left(\frac{\alpha_{s}}{\alpha}\right)^{2}\left\langle\sigma_{11} v\right\rangle$. Show that

$$
\left\langle\sigma_{e f f} v\right\rangle=\left\langle\sigma_{11} v\right\rangle\left(\frac{1+\frac{\alpha_{s} \omega}{\alpha}}{1+\omega}\right)^{2}
$$

with $\omega=(1+\Delta)^{3 / 2} e^{-\Delta x} g_{2} / g_{1}, \Delta=\left(m_{2}-m_{1}\right) / m_{1}$. How close do the masses of $\chi_{1}$ and $\chi_{2}$ have to be for the presence of $\chi_{2}$ to have an appreciable effect on the relic abundance?

Question 15. Co-annihilation $b$. Consider another interesting limit where there is little actual co-annihilation but the second state still impacts the final abundance, namely $\sigma_{12} \ll \sigma_{11}, \sigma_{22}$. Show that this can lead to a smaller effective cross section, therefore larger relic abundance. Explain what is going on physically.

\subsection{Direct detection}

As discussed in Section 1.1 there is strong evidence that our Galaxy is surrounded by a large halo of non-relativistic DM. Since WIMPs have couplings to SM particles there is a rate for the halo WIMPs to scatter off atoms in terrestrial laboratories [70]. Looking for such events is the purpose of direct DM detection which now consists of many experiments located around the globe using different target elements and detection technologies to search for this signal e.g. (Super)CDMS [71, 72], CoGeNT [73], COUPP [74], CRESST [75], DAMA/LIBRE [76], KIMS [77], XENON(1T)[78, 79]. For a WIMP of weak scale mass the scattering is off nuclei, but for lighter masses electron scattering is the dominant process. We focus on nuclear scattering here, for more discussion of electron scattering and direct detection in general see Tongyan Lin's lectures in this volume. For a review of the techniques presented in this section see [80]. 
The first input needed to determine the scattering rate is the amount of dark matter in our vicinity [81, 82]. While there are various models of how DM is distributed, with respect to radius, in our Galaxy most agree on the DM density in our neighbourhood, partially because we assume about $50 \%$ uncertainty on the value! Historically the local DM density was taken to be $\rho \sim 0.3 \mathrm{GeV} \mathrm{cm}^{-3}$ and this is still what is extracted from analysing the motions of local stars [83]. Recently global fits [84] have found $\rho \sim 0.4 \mathrm{GeV} \mathrm{cm}^{-3}$, but direct detection results are still mostly presented assuming $\rho \sim 0.3 \mathrm{GeV} \mathrm{cm}^{-3}$.

Question 16. DM Flux If Dark Matter is a $100 \mathrm{GeV}$ WIMP approximately how many Dark Matter particles pass through your hand every second? Compare this to the number of solar neutrinos.

The second input we need to know is how this DM is moving in our Galaxy. Here we cannot use measurements of DM's gravitational action on stars to infer its speed, so instead we turn to general arguments and N-body simulations [85]. An isotropic spherical halo is expected to have a speed distribution that is Maxwell-Boltzmann in form, $f(v) \sim e^{-v^{2} / v_{0}^{2}}$. This is qualitatively supported by N-body simulations but there can be significant quantitative deviations, especially at high DM speeds [86]. It should be noted that N-body simulations do not have the capability to resolve the halo structure on the length scales probed by our experiments, they can only make average statements. So the first true determination of the DM halo will be through direct detection of DM itself. This has led to recent interest in "halo-independent" techniques [87, 88]. For the purposes of interpreting direct detection results, and for these lectures, it is common to make the simplifying assumption that the local DM distribution follows the standard halo model (SHM) [89], the spherical cow of DM distributions,

$$
(\mathrm{SHM}) \quad \rho=0.3 \mathrm{GeV} \mathrm{cm}^{-3}, \quad f(\vec{v})=N e^{-|\vec{v}|^{2} / v_{0}^{2}} \Theta\left(v_{\mathrm{esc}}-|\vec{v}|\right)
$$

where the velocities are in the Galactic frame, rather than the frame of the Earth, and the normalisation factor $N$ is fixed by requiring $\int d^{3} v f(\vec{v})=1$. Typically values for the circular rotation speed and the escape speed are $v_{0}=220 \mathrm{~km} \mathrm{~s}^{-1}$ and $v_{\mathrm{esc}}=544 \mathrm{~km} \mathrm{~s}^{-1}$. It should be emphasised that the SHM is only an approximation to the real situation. There will be corrections coming from observed Galactic substructure e.g. [90].

Finally, searches for DM take place on the Earth so we need to determine the incoming DM speed distribution in the Earth frame. Since all motions are non-relativistic this is done through a Galilean boost by the Earth's velocity in the Galactic frame, $\vec{u}(t)$. Thus, the speed distribution in the Earth's frame is $f_{\oplus}(\vec{v}, \vec{u})=f(\vec{v}+\vec{u})$. The motion of the Earth is a combination of the Sun's motion in the Galaxy and the Earth's motion around the Sun, $\vec{u}(t)=\vec{v}_{\otimes}+\vec{u}_{\oplus}(t)$, the later varies over a sidereal year. The direction of these motions [91] is such that the magnitude of $\vec{u}$ varies by $\sim 10 \%$ over a year.

The kinematics of the DM-nucleus collision are straightforward. In the lab frame a nonrelativistic WIMP of mass $m$ and incoming speed $\vec{v}$ collides with a stationary nucleus of mass $m_{N}$ which recoils with energy $E_{R}$ and momentum $\vec{q}$. In the centre-of-momentum frame, where $\vec{v}$ is the relative velocity of the two incoming particles, the incoming momentum is $\vec{p}=\mu \vec{v}$ and the outgoing 
momentum is $\vec{p}^{\prime}$. We introduce the reduced mass defined with respect to the incoming particles,

$$
\mu \equiv \frac{m_{\chi} m_{N}}{m_{\chi}+m_{N}} .
$$

The recoil energy of the collision is $E_{R}=q^{2} / 2 m_{N}^{\prime}$ with

$$
q^{2}=p^{2}+p^{2}-2 p p^{\prime} \cos \theta_{\text {com }} \text {. }
$$

In general the outgoing WIMP and nucleus may be of different mass than the incoming particles, with splitting $\delta_{\chi} \equiv m_{\chi}^{\prime}-m_{\chi}$ and $\delta_{N} \equiv m_{N}^{\prime}-m_{N}$, respectively. The recoil of energy $E_{R}$, speed $v$ and $\cos \theta_{\text {lab }}$ are related by,

$$
\frac{v^{2}}{2} \delta_{\chi} \frac{m_{\chi}}{m_{\chi^{\prime}}}-v \frac{m_{\chi}}{m_{\chi^{\prime}}} \sqrt{2 m_{N^{\prime}} E_{R}} \cos \theta_{\mathrm{lab}}-\left[E_{R}\left(1+\frac{m_{N^{\prime}}}{m_{\chi^{\prime}}}\right)+\delta_{\chi}+\delta_{N}\right]=0 .
$$

Defining $\delta \equiv \delta_{\chi}+\delta_{N}$ and assuming $\delta>0$, we can safely perform an expansion in $\delta / m \ll 1$ to obtain

$$
v_{\min }=\frac{1}{\sqrt{2 m_{N} E_{R}}}\left(\frac{m_{N} E_{R}}{\mu}+\delta\right) .
$$

Taking $\delta_{N} \rightarrow 0$ this is the well-known result for inelastic dark matter (iDM) [59, 92, 93]. For most SUSY WIMP models the WIMP candidate undergoes only elastic scattering and $\delta=0$. Inserting typically WIMP masses and speeds of DM in our halo we see that the recoil momentum is $q \sim$ $10-100 \mathrm{MeV}$, the recoil energy is $E_{R} \sim 1-10 \mathrm{keV}$, and that the energy deposited by the signal is very feeble. This means that the scattering takes place off the whole nucleus at low momentum exchange but may be off only a fraction of the nucleons at high $q$. This loss of coherence will be taken into account through a nuclear form factor.

The last remaining input we need is the DM-nucleus scattering cross section. The WIMP couples to quarks and gluons which are components of the nucleus and we must match these fundamental couplings to interactions with protons and neutrons using nucleon matrix elements. From there we calculate the WIMP-nucleus scattering amplitude, which introduces nuclear form factors. Throughout this process we should recall that the scattering is non-relativistic and the momentum exchange is small. For the case of SUSY WIMPs, and many other models, where the mediators are heavy and can be integrated out, the matching is done between higher dimension operators in a WIMP EFT, making the results widely applicable.

In SUSY the WIMP is a Majorana fermion and so does not have vector interactions with quarks, instead the interactions are either scalar, through Higgs or squark exchange, or spindependent, through $Z$ exchange or squark exchange. Some example processes contributing to direct detection cross sections for WIMPS are shown in Figure 7. The tree-level Higgs exchange is dominated by the strangeness content of the nucleon [94] while the loop diagrams with Higgs exchange involve heavy quark loops.

The spin-dependent processes will lead to effective operators of the form,

$$
\mathscr{L}_{\text {SD }}=\frac{\kappa}{\Lambda^{2}} \bar{\chi} \gamma^{\mu} \gamma_{5} \chi \bar{q} \gamma_{\mu} \gamma_{5} q
$$

while the spin-independent gives effective operators include

$$
\mathscr{L}_{\mathrm{SI}}=\frac{\lambda_{1}}{\Lambda^{2}} \bar{\chi} \chi \bar{q} q+\frac{\lambda_{2}}{\Lambda^{3}} \bar{\chi} \chi G_{\mu \nu} G^{\mu \nu} .
$$



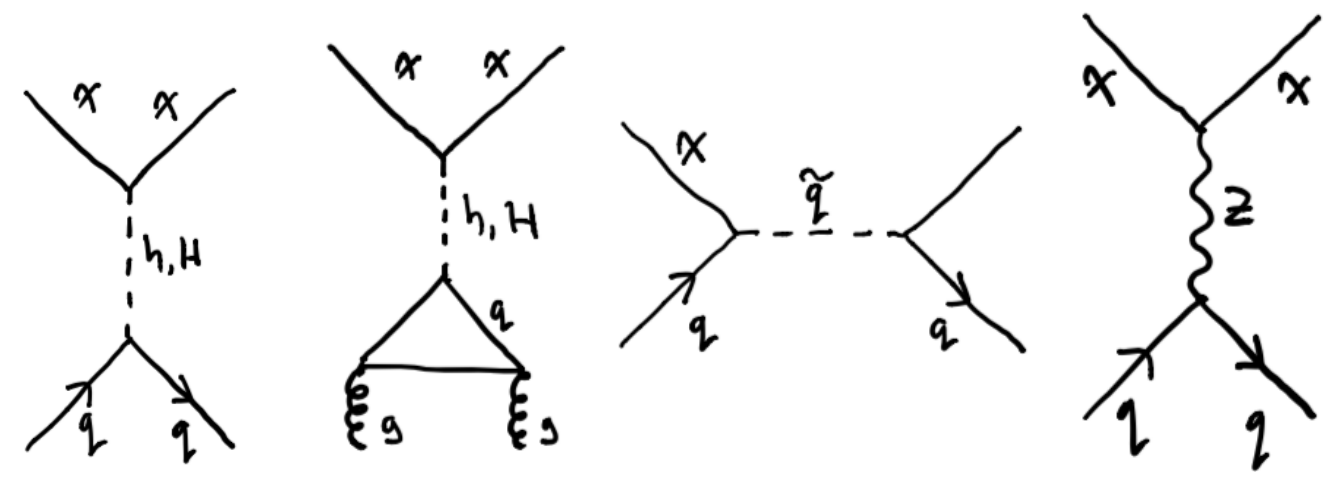

Figure 7: Some example diagrams for a neutralino scattering off components of a nucleon. The first two diagrams contribute to spin-independent scattering the third to both spin-independent and spin-dependent and the last only to spin-dependent.

The coefficients in the above couplings are determined by calculating the relevant Feynman diagrams. Once these are known we match the quark or gluon level operator, e.g. $\bar{q} \Gamma q$, to the nucleon operators by calculating the nucleon matrix elements $e . g .\langle n|\bar{q} \Gamma q| n\rangle$. Finally we match to the nuclear system. At zero momentum transfer this is done by evaluating the nucleon-level operators in the nucleus but at higher momentum transfer we must also include a form factor which accounts for the fact that the WIMP only resolves a piece of the nucleus, of size $|\vec{q}|^{-1}$. For spin-dependent operators the nucleon spin operator evaluates (at $\vec{q}=0$ ) to the spin of the nucleus, which is the spin of the unpaired nucleons and is typically not large. Evaluating the spin-independent nucleon operators on the nucleus just count the number of protons and neutrons. Thus, spin-independent couplings acquire a coherent enhancement of $Z^{2},(A-Z)^{2}$, or $A^{2}$, from coupling to all protons, neutrons, or both, respectively. This means that constraints on spin-independent couplings are stronger than spin-dependent and benefit from using target atoms of large $A$. For this reason we will now concentrate on the spin-independent case.

We parametrize the scalar WIMP-nucleon operators after matching to the proton and neutron level operators as $f_{p} \bar{\chi} \chi \bar{p} p+f_{n} \bar{\chi} \chi \bar{n} n$. These in turn map to nuclear-level operators as $\left(Z f_{p}+(A-Z) f_{n}\right) \bar{\chi} \chi \bar{N} N$. Finally, we can carry out a usual tree level field theory calculation for $\chi-N$ scattering and take the non-relativistic limit to arrive at the differential cross section,

$$
\frac{d \sigma}{d E_{R}}=\frac{m_{N}}{2 \pi v^{2}}\left(Z f_{p}+(A-Z) f_{n}\right)^{2}\left|F\left(E_{R}\right)\right|^{2}
$$

where we have included the form factor. To facilitate comparisons of results from detectors using different target materials this is often expressed in terms of a per-nucleon scattering cross section. The relationship between these can be found by ignoring the form factor in (5.16) and calculating the total cross section $\sigma_{N}=\mu_{N \chi}^{2}\left(Z f_{p}+(A-Z) f_{n}\right)^{2} / \pi$, where $\mu_{N \chi}=m_{N} m_{\chi} /\left(m_{N}+m_{\chi}\right)$ is the WIMP-nucleus reduced mass. A similar calculation for the per nucleon cross section would replace $\mu_{N \chi}$ with $\mu_{n \chi}$ and not have the $A$ enhancement. Thus,

$$
\sigma_{N}=\frac{\left(Z f_{p}+(A-Z) f_{n}\right)^{2}}{f_{p}^{2}} \frac{\mu_{N \chi}^{2}}{\mu_{p \chi}^{2}} \sigma_{p} .
$$




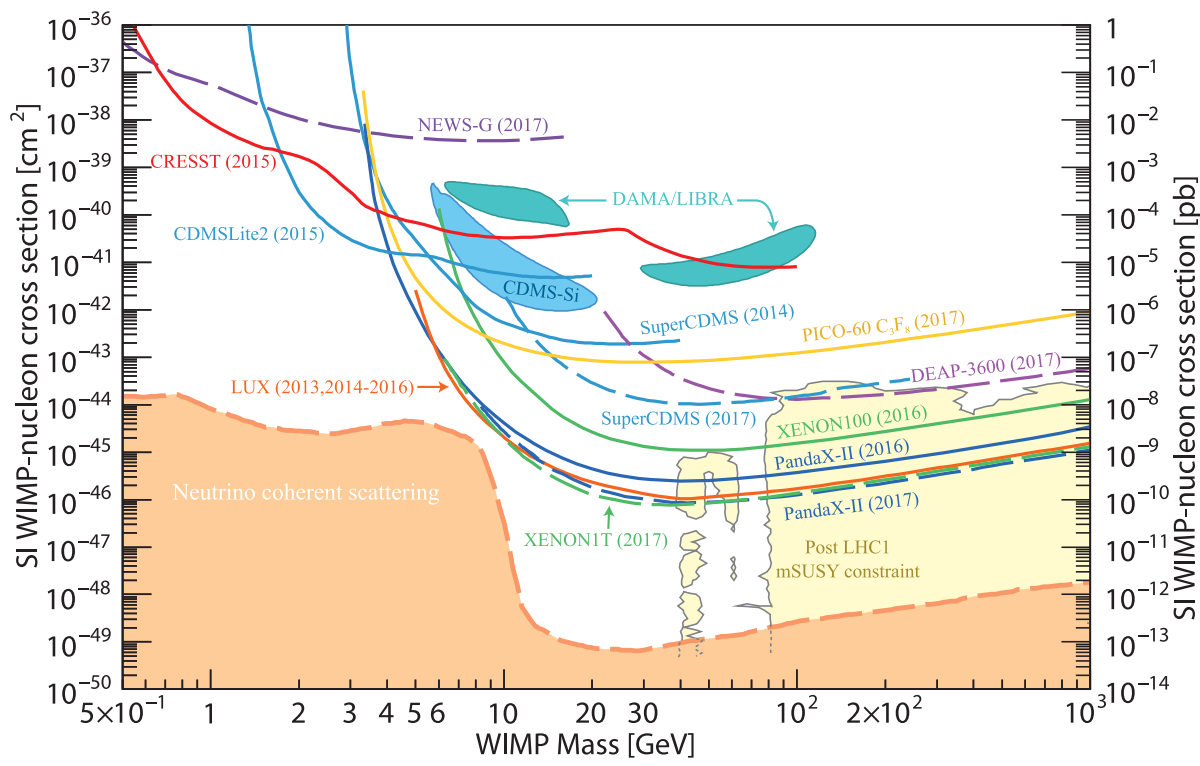

Figure 8: Status of spin-independent direct detection searches, taken from the PDG [95].

The differential DM scattering rate is determined by putting all of the above discussion together,

$$
\frac{d R}{d E_{R}}=N_{T} \frac{\rho}{m} \int_{v_{\min }}^{v_{\max }} d^{3} v f_{\oplus}(\vec{v}, \vec{u}(t)) \frac{d \sigma|\vec{v}|}{d E_{R}}
$$

Of course, what experiments actually observe will be this theoretical prediction convolved with the detector response, efficiencies etc, which we will not concern ourselves with here ${ }^{15}$. At present the majority of the direct detection experiments see no DM-like events, with the notable (and long standing) exception of DAMA/LIBRA [76]. By comparing the lack of observation with (5.18) constraints can be placed on the WIMP-nucleon cross section. The present status is summarised in Figure 8. At WIMP masses of around $10 \mathrm{GeV}$ the bounds are approaching the "neutrino floor", an irreducible background coming from coherent neutrino scattering, while at higher masses we have some way to go. At low masses the background comes from solar neutrinos and at higher masses from a combination of (predominantly) atmospheric and diffuse supernova neutrinos.

Question 17. Minimal DM The "original" WIMP was a particle coupled to the $Z$. Show that the direct detection scattering cross section off a nucleus ${ }_{Z}^{A} N$ through $Z$ exchange for a Dirac fermion with hypercharge $Y$ is

$$
\sigma(\chi N \rightarrow \chi N)=\frac{G_{F}^{2} M_{N}^{2}}{2 \pi} Y^{2}\left(N-\left(1-4 s_{W}^{2}\right) Z\right)^{2}
$$

\footnotetext{
${ }^{15}$ In the words of Yogi Berra, "In theory, there is no difference between practice and theory. In practice, there is".
} 
Question 18. A model for Inelastic Dark Matter By decomposing a Dirac 4-spinor as two 2-spinors,

$$
\psi=\left(\begin{array}{l}
\eta \\
\bar{\xi}
\end{array}\right)
$$

Determine the mass eigenstates for a mass term of the form $m \bar{\psi} \psi+\frac{\delta_{1}}{2}(\eta \eta+\bar{\eta} \bar{\eta})+\frac{\delta_{2}}{2}(\xi \xi+\bar{\xi} \bar{\xi})$. Assume there is a coupling to a vector boson, $A_{\mu}$, of the form $\bar{\psi} \gamma^{\mu} A_{\mu} \psi$. Determine what this is in terms of the mass eigenstates.

Question 19. Inelastic Dark Matter in Direct Detection The rate equation for direct detection, ignoring experimental effects like efficiencies, thresholds etc, is

$$
\frac{d R}{d E}=\frac{N_{T} m_{N} \rho_{\chi}}{2 \mu_{N \chi}^{2} m_{\chi}} \int_{v_{\min }}^{v_{e s c}} d^{3} v \frac{f\left(v, v_{\oplus}\right)}{v} \sigma_{N} F^{2}(E)
$$

Use the Standard Halo Model to draw the shape of the recoil spectrum (ignore the form factor) for a) elastic DM and b) inelastic DM. Is the modulation expected to be larger or smaller for iDM?

Question 20. Modulation signals Consider the Earth's daily rotation and its orbit around the Sun. Compare these modulating velocities to the Sun's motion in the Galaxy. Estimate how big the daily modulation signal at DAMA would be.

We now return briefly to the origin of the WIMP-SM couplings in SUSY. Consider the situation with heavy squarks and the Higgs sector in the decoupling limit $\left(m_{H, A} \gg m_{h}\right.$, so that the only mediators contributing to the direct detection rate are the SM higgs, $h$, and the $Z$ boson. Recall that the more strongly constrained spin-independent rate comes from $h$ exchange and spin-dependent from $Z$ exchange. For effective couplings,

$$
\frac{c_{h \chi \chi}}{2} h\left(\chi \chi+\chi^{\dagger} \chi^{\dagger}\right)+c_{Z \chi \chi} \chi^{\dagger} \bar{\sigma}^{\mu} \chi Z_{\mu}
$$

the approximate scattering cross sections are

$$
\sigma_{\mathrm{SI}} \sim 10^{-42} c_{h \chi \chi}^{2} \mathrm{~cm}^{2}, \quad \sigma_{\mathrm{SD}} \sim 10^{-37} c_{Z \chi \chi}^{2} \mathrm{~cm}^{2} .
$$

The Higgs-neutralino, $c_{h \chi \chi}$, coupling comes from the Kähler potential and is off-diagonal in field space, $H^{\dagger} e^{V} H \rightarrow h \tilde{h} \tilde{W}, h \tilde{h} \tilde{B}$, so pure WIMP states do not have a tree-level SI scattering cross section. For non-pure states it is still possible to have a small coupling at a so-called "blind spot" [96]. If the neutralino has a coupling to the Higgs it has a contribution to its mass from the Higgs vev, $\partial m_{\chi} / \partial v=c_{h \chi \chi}$. First note that if $c_{h \chi \chi}=0$ then the WIMP mass is the same as it would be with no electroweak symmetry breaking, namely $m_{\chi}=M_{1}, M_{2}$, or $-\mu$. Secondly, recall that the neutralino mass is determined by finding the eigenvalues of the neutralino mass matrix (4.21), i.e. solutions to the equation $\operatorname{det}\left(M_{N}-m_{\chi} \mathbb{1}\right)=0$. Taking the derivative of the eigenvalue equation w.r.t the vev we find the Higgs-neutralino coupling is zero if

$$
\left(m_{\chi}+\mu \sin 2 \beta\right)\left(m_{\chi}-\frac{1}{2}\left[M_{1}+M_{2}+\cos 2 \theta_{W}\left(M_{1}-M_{2}\right)\right]\right)=0 .
$$




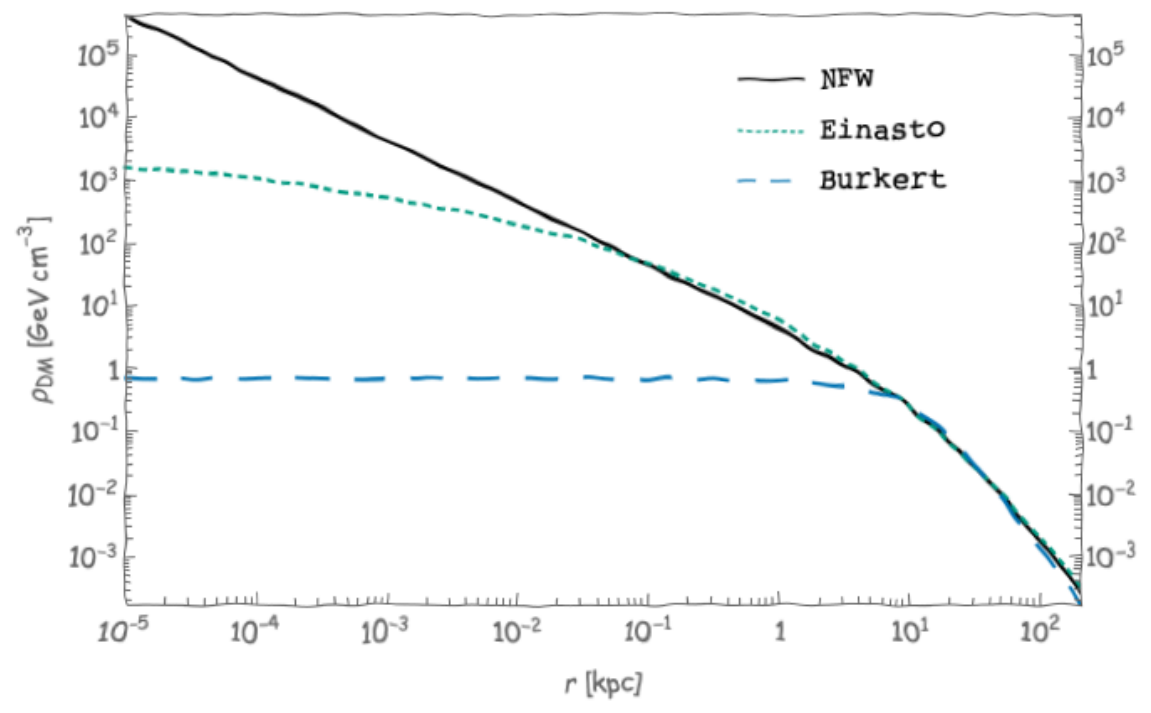

Figure 9: The shape of NFW, Einasto and Burkert DM profiles. The parameters for each have been chosen so that $\rho(r=0.8 \mathrm{kpc})=0.3 \mathrm{GeV} \mathrm{cm}^{-3}$.

Thus, there is a blind spot in parameter space for SI direct detection if $m_{\chi}=M_{1}, M_{2},-\mu$ if $m_{\chi}+$ $\mu \sin 2 \beta=0$ (which will require certain sign choices between parameters) or at $m_{\chi}=M_{1}=M_{2}$. Similarly, there is a blind spot for SD couplings. Only the Higgsino couples to the $Z$ boson so the WIMP- $Z$ coupling, $c_{Z \chi \chi}$, must arise from the Higgsino component. For $\tan \beta=1$ there is an enhanced $H_{u} \leftrightarrow H_{d}$ symmetry and the $Z$ coupling is off diagonal between $\frac{1}{\sqrt{2}}\left(\widetilde{H}_{u}^{0} \pm \widetilde{H}_{d}^{0}\right)$, as for the pure Higgsino state.

These blind spots will be moved around slightly by loop corrections but will still exist. There can also be other blind spots, for instance if we move away from the decoupling limit there could be cancellation between $h$ and $H$ exchange [97]. Although these blind spots appear to be bad news for direct detection it is still possible to discover DM in these scenarios by looking in complementary places e.g. the LHC, indirect detection, and SD detection for SI blind psots [98].

Question 21. Non-relativistic limits Consider fermionic DM $\chi$ coupled to SM fermions $f$. What are the low velocity limits of the interactions (a) $\bar{\chi} \chi \bar{f} f$, (b) $\bar{\chi} \gamma_{\mu} \gamma_{5} \chi \bar{f} \gamma^{\mu} \gamma_{5} f$, and (c) $\bar{\chi} \gamma_{\mu} \gamma_{5} \chi \bar{f} \gamma^{\mu} f ?$

\subsection{Indirect detection}

The thermal production of DM provides a compelling "origin story" for the main protagonist of these lectures. We will now discuss searching for DM through the same annihilation processes that determined its abundance, but taking place now rather than a few instants after the big bang. There are many reviews on this topic, a very comprehensive presentation of the field is given in the "cookbook" [99], and a more detailed account of the present status, including the Galactic Centre Excess (GCE), can be found in the lectures by Hooper in this volume [100]. 
The present day annihilation of WIMP DM leads to energetic SM particles, in particular antiparticles, in the cosmos - which is a signal that can be searched for. The rate scales as $\sim \rho^{2}$ so it is most productive to look for the appearance of high energy antiparticles or photons in places where there is a high DM density. For a sense of how DM is distributed in our Galaxy see Figure 9, where three commonly assumed DM profiles are shown. The "cuspy" profiles of NFW [101] and Einasto $[102,103]$ have a large increase in density towards the Galactic centre, whereas the "cored" profile of Burkert [104] has less of an enhancement. The functional form of each of these profiles is

$$
\begin{aligned}
\rho_{\mathrm{NFW}} & =\frac{\rho_{s}}{\frac{r}{r_{s}}\left(1+\frac{r}{r_{s}}\right)^{2}}, \\
\rho_{\text {Einasto }} & =\rho_{s} \exp \left(-\frac{2}{\alpha}\left[\frac{r^{\alpha}}{r_{s}^{\alpha}}-1\right]\right), \\
\rho_{\text {Burkert }} & =\frac{\rho_{s}}{\left(1+\frac{r}{r_{s}}\right)\left(1+\frac{r^{2}}{r_{s}^{2}}\right)},
\end{aligned}
$$

where in all cases the parameters associated with the profiles have been chosen to reproduce the observed density at the Sun's radius [99].

Regardless of which profile is closer to the truth it is clear that the Galactic centre is an interesting place to look for DM annihilation. Our Galaxy also has a set of smaller companion satellites which are rich in dark matter and relatively poor in stars. These dwarf galaxies also provide an opportunity to search for the DM annihilation products. Although further away than the Galactic centre they have fewer other sources of high energy particles so offer complementary systematics to the Galactic centre.

After deciding where to look one must decide what to look for. As already mentioned, antiparticles are not common in the Galaxy but DM annihilation is symmetric between particle and anti-particle production. Thus, we look for positrons, antiprotons, anti-deuterium, etc in high energy cosmic ray fluxes. The source flux, $Q$, of these particles is determined by the abundance of $\mathrm{DM}$ and the annihilation modes

$$
Q=\frac{1}{2}\left(\frac{\rho}{m_{\chi}}\right)^{2} \sum_{f}\langle\sigma v\rangle_{f} \frac{d N_{e^{ \pm}}^{f}, p \bar{p} . .}{d E},
$$

here $d N_{i} / d E$ is the energy spectrum of charged particle $i$ that comes out of DM annihilation. This is often determined through computer programs like PYTHIA [105] since the long-lived charged particles are often the products of a complicated decay chain often involving showering and hadronisation.

The propagation of these charged particles from source to detectors at (or in an orbit of) Earth is complicated and is governed by a diffusion-loss equation. Charged particles move through the Galaxy diffusing on inhomogeneities in the magnetic field and losing energy through synchrotron radiation, inverse Compton scattering on CMB and starlight photons, and colliding with gas. Correctly modeling all of this requires many parameters which are not all well known. The discussion of the details of this are beyond these lectures, but are well covered in the lectures of Hooper [100]. 


\begin{tabular}{l|l|c} 
Annihilation mode & \multicolumn{1}{|c}{ Comments } & Example experiments \\
\hline \hline$p \bar{p}, e^{+} e^{-}, \ldots$ & $\begin{array}{l}\text { Antimatter in cosmic rays. Non-pointing, propa- } \\
\text { gation determined by diffusion-loss equation. }\end{array}$ & PAMELA, AMS2, Fermi \\
$v \bar{v}$ & $\begin{array}{l}\text { Pointing. Hard to detect. } \\
\text { Pointing. Spectrum is a line }\left(E_{\gamma} \approx m / 2\right) . \text { Low }\end{array}$ & $\begin{array}{l}\text { Icecube, Antares, SuperK } \\
\text { Fermi, HESS }\end{array}$ \\
$\gamma \gamma$ & $\begin{array}{l}\text { rate (loop process). } \\
\text { PMSM } \rightarrow \gamma+X\end{array}$ & $\begin{array}{l}\text { Pointing. Energy spectrum is continuum with } \\
\text { edge. Complicated backgrounds. }\end{array}$ \\
e.g. $\pi^{0}, b, \tau$ &
\end{tabular}

Table 4: Summary of ways in which DM annihilation can be searched for.

Instead, we briefly turn to the case of annihilation to photons where transit effects are less important and futhermore there is an interesting excess in the Galactic centre [106, 107]. A summary of the various approaches is given in Table 4 .

The differential flux of photons produced in DM-annihilation coming from direction $\psi$ is given by

$$
\frac{d \Phi}{d E_{\gamma}}\left(E_{\gamma}, \psi\right)=\frac{1}{4 \pi} \int_{\Delta \Omega} \int_{\text {l.o.s }} d l \frac{1}{2}\left(\frac{\rho(r(l, \psi))}{m_{\chi}}\right)^{2} \sum_{i}\left\langle\sigma_{i} v\right\rangle \frac{d N_{i}}{d E_{\gamma}}
$$

The energy spectrum of photons per annihilation is $d N_{i} / d E_{\gamma}$. The line-of-sight integral, denoted as $J=\int_{1.0 . \mathrm{s}} d l \rho(l)^{2}$, determines which places are the best to look for these photons. Note that when observing solid angle $\Delta \Omega$ the amount of DM being observed grows with the square of the distance from the observer, but the fraction of the annihilation flux that reaches the observer also drops as this distance squared. Thus, for constant DM density all distances are equally important. Ideally, we would like to look at high density sites that are nearby. As discussed before, this means the the Galactic centre and dwarf galaxies.

The photon spectrum one expects depends on the model. A neutralino has no direct coupling to photons but can have a loop induced annihilation to a pair of photons, for instance through a loop of $W^{ \pm}$'s, or to a photon and another weak boson, generically denoted $\phi$. This leads to the very distinctive signature of a photon line at $E_{\gamma}=m_{\chi}$ or $E_{\gamma}=m_{\chi}\left(1-m_{\phi}^{2} / 4 m_{\chi}^{2}\right)$, but the rate is very small. Alternatively, photons can be generated when DM annihilates to SM states which subsequently decay or shower and hadronise. For instance if DM annihilates to a pair of $W$ 's these in turn decay to a mixture of quarks and leptons. The quarks shower and hadronise which makes boosted $\pi^{0}$ 's which in turn decay to photons. The spectrum of these photons is not as distinctive as a line, instead it is a broad spectrum which ends at $m_{\chi}$, however it is something that can be searched for. Annihilations into other SM states, such as $b \bar{b}, \tau^{+} \tau^{-}$give qualitatively similar spectra although the quantitative details differ. Intriguingly, an excess of photons from the Galactic centre has been observed $[106,107]$ and appears to be well fit by a DM particle of $40-70 \mathrm{GeV}$ annihilating to $b \bar{b}$ with annihilation cross section comparable to that expected for a thermal relic, $\langle\sigma v\rangle \sim 10^{-26} \mathrm{~cm}^{3} \mathrm{~s}^{-1}$. Finally, in dark sector models with a dark sector mediator $\phi$ coupled to DM there can be annihilations of the form $\chi \chi \rightarrow \phi \phi \rightarrow 4 \gamma$ that gives a distinctive box-like spectrum for the photons. This spectrum is flat between the end points $E_{ \pm}=m_{\chi}\left(1 \pm \sqrt{1-m_{\phi}^{2} / m_{\chi}^{2}}\right)$. These 


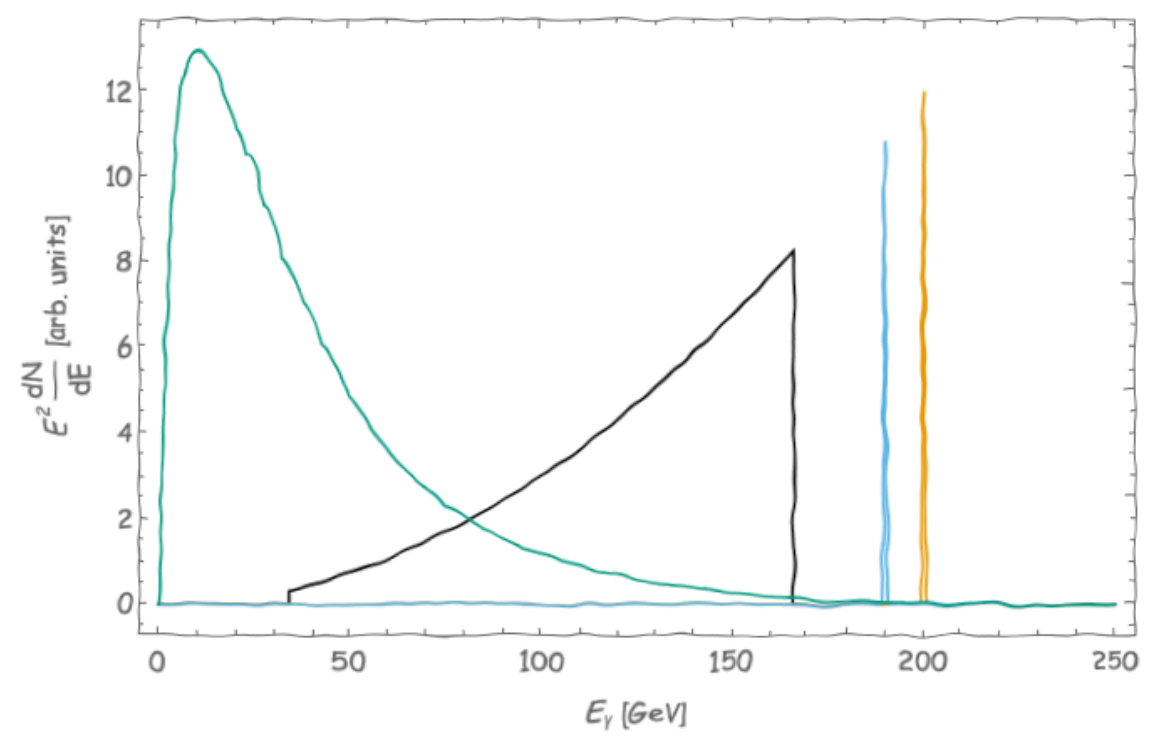

Figure 10: The photon spectra (in arbitrary units) expected for $200 \mathrm{GeV}$ DM annihilating in various modes. From left left to right these modes are: $\chi \chi \rightarrow W^{+} W^{-}, \chi \chi \rightarrow \phi \phi \rightarrow \gamma \gamma\left(m_{\phi}=150 \mathrm{GeV}\right), \chi \chi \rightarrow Z \gamma, \chi \chi \rightarrow \gamma \gamma$.

example spectra are shown in Figure 10. Note that it is typical to show spectra weighted by $E^{2}$, i.e. to plot $E^{2} d N / d E$ since backgrounds scale as $E^{-(2-3)}$.

\subsection{Capture in the Sun}

Another location where there may be a large amount of DM is at the centre of celestial bodies, e.g. the Sun [108], Earth [109], or large planet. If a DM particle from the halo scatters with a nucleus in one of these bodies and loses enough momentum that its speed falls below the escape speed it will be captured and will, through subsequent scatters and loss of energy, sink to the centre of the gravitational potential. Overtime a DM overdensity will build up and there will be an enhanced annihilation rate. In order to be able to observe this annihilation the annihilation products must be able to reach us from the centre of the object, which means that the DM annihilation must lead to high energy neutrinos. For concreteness we focus on capture in the Sun and outline the capture of DM and subsequent neutrino production below. As for indirect detection there is a comprehensive "cookbook" that outlines in detail the recipe for computing the DM signal [110].

There are three competing processes that determine the abundance of DM in the Sun: DM capture, its annihilation, and its evaporation. Denoting the rate for each process as $\Gamma_{i}$ the evolution of DM in the Sun is

$$
\frac{d N}{d t}=\Gamma_{\text {capt }}-2 \Gamma_{\text {ann }}-\Gamma_{\text {evap }} .
$$

If the DM is thermalised at the centre of the Sun, where the temperature is $T_{\odot}=1 \mathrm{keV}$, then for light DM its thermal motion will be above the escape speed, $v_{\mathrm{esc}} \sim 1400 \mathrm{~km} \mathrm{~s}^{-1}$, and the DM will evaporate. A careful analysis shows that for DM heavier than $\sim 4 \mathrm{GeV}$ there is not enough DM in 
the high speed tail of the Boltzmann distribution for evaporation to take place on timescales shorter than the age of the Sun and $\Gamma_{\text {evap }}$ can be ignored.

To make progress we will make some simplifying assumptions: the Sun is a uniform density $\rho_{\odot} \approx 150 \mathrm{~g} \mathrm{~cm}^{-3}$ and that the DM has thermalised in the Sun and therefore its number density is given by $n=n_{0} e^{-m \phi(r) / T}$ where $\phi(r)=\int_{0}^{r} d x G_{N} M(x) / x^{2}$ is the gravitational potential in the Sun. For the constant density assumption the contained mass $M$ has a simple form, $M(r)=4 \pi r^{3} \rho_{\odot} / 3$. Thus, one finds,

$$
n=n_{0} e^{-r^{2} / r_{\mathrm{DM}}^{2}}, \quad r_{\mathrm{DM}}=\left(\frac{3 T_{\odot}}{2 \pi G_{N} \rho_{\odot} m_{\chi}}\right)^{1 / 2} \approx 0.01 \sqrt{\frac{100 \mathrm{GeV}}{m_{\chi}}} R_{\odot} .
$$

The DM is confined to a very small sphere at the centre of the Sun. Any annihilation products will point back to this small point source, which will be a useful way to remove isotropic backgrounds. Knowing the DM distribution we can calculate the annihilation rate,

$$
\Gamma_{\mathrm{ann}}=\frac{1}{2} \int_{\text {Sun }} d^{3} r n^{2}\langle\sigma v\rangle \Rightarrow \Gamma_{\mathrm{ann}}=\frac{1}{2}\langle\sigma v\rangle\left(\frac{G_{N} m_{\chi} \rho_{\odot}}{3 T_{\odot}}\right)^{3 / 2} N^{2} \equiv \frac{1}{2} C_{\mathrm{ann}} N^{2} .
$$

With the expression for the annihilation rate (5.32) we can now solve (5.30), ignoring the effects of evaporation and assuming DM only captures through scattering off the Sun not through previously captured DM, to find

$$
N=\sqrt{\frac{\Gamma_{\text {capt }}}{C_{\text {ann }}}} \tanh \frac{t}{\tau} \stackrel{t \gg \tau}{\longrightarrow} \sqrt{\frac{\Gamma_{\text {capt }}}{C_{\text {ann }}}},
$$

where $\tau^{-2}=\Gamma_{\text {capt }} C_{\text {ann }}$. It turns out that the age of the Sun is greater than the relevant timescale $\tau$ and the DM has reached equilibrium. This is not true for the Earth. Inserting the result of (5.33) in (5.32) we find that in the equilibrium the annihilation rate is set by only the capture rate; DM annihilates away as fast as it arrives.

It remains to determine $\Gamma_{\text {capt }}$, which is a complicated calculation $[111,112,108]$ and is given by,

$$
\Gamma_{\text {capt }}=n_{\chi} \sum_{i} \sigma_{i} \int_{0}^{R_{\odot}} d r 4 \pi r^{2} n_{i}(r) \int_{0}^{\infty} d v 4 \pi v^{2} f_{\odot}(v) \frac{v^{2}+v_{\odot \mathrm{esc}}(r)^{2}}{v} P_{\text {cap }}
$$

where $P_{\text {cap }} \sim\left(\Delta E_{\max }-\Delta E_{\min }\right) / \Delta E_{\max }$ is the capture probability and $\Delta E_{\min , \max }$ is the minimum (maximum) energy loss necessary for capture ${ }^{16}$. In (5.34) $f_{\odot}(v)$ is the DM speed distribution in the Sun's frame and $v_{\odot \text { esc }}(r)$ is the speed necessary to escape from radius $r$ inside the Sun. The capture off all elements in the Sun is summed in (5.34), but it is dominated by SD scattering off hydrogen. Using models for the distribution of elements within the Sun, assuming a Maxwell-Boltzmann velocity distribution and making other simplifying assumptions [110] leads to an approximate, but more illuminating, expression for the capture rate

$$
\Gamma_{\text {capt }} \approx 6 \times 10^{26} \mathrm{~s}^{-1}\left(\frac{\rho}{0.3 \mathrm{GeVcm}^{-3}}\right)\left(\frac{100 \mathrm{GeV}}{m_{\chi}}\right)^{2}\left(\frac{270 \mathrm{~km} \mathrm{~s}^{-1}}{v_{0}}\right)\left(\frac{\sigma_{\mathrm{SD}}+1200 \sigma_{\mathrm{SI}}}{\mathrm{pb}}\right) .
$$

\footnotetext{
${ }^{16}$ These minimum and maximum energies are given by $\Delta E_{\max }=4 m_{N} m_{\chi} /\left(m_{N}+m_{\chi}\right)^{2}$ and $\Delta E_{\min }=v^{2} /\left(v^{2}+v_{\odot \text { esc }}^{2}\right)$. As expected this favours capture of slow moving DM.
} 
Given the direct detection constraints on spin-independent and spin-dependent scattering cross sections the spin-dependent scattering off hydrogen (and to a lesser extent nitrogen) are the most important for capture.

The final step in getting to the signal of DM annihilation in the Sun is to determine the spectrum of annihilation products. The only particle species that can make it from the centre of the Sun to Earth is neutrinos, and their spectrum will depend upon the annihilation process. In a similar fashion to indirect detection, Section 5.3, this can be determined by running codes like PYTHIA. The only complication is that the outgoing SM particles are moving in a material. These effects must be modelled as well [110]. In most models the DM does not annihilate to a pair of neutrinos so there is no line, instead there is a continuum coming from decays of the annihilation products (e.g. $\chi \chi \rightarrow W^{+} W^{-} \rightarrow \ell v+\ldots$ ). Charged lepton decays involve a neutrino, but the muon will stop before decaying, while the tau decays in flight and provides a higher energy neutrino. In addition to the annihilation products propogating through solar matter, the neutrinos, once produced, also have to transit through the Sun and intervening space to the Earth. In doing so they will oscillate and this must also be taken into account, but a discussion of this is beyond these lectures. Finally, upon arrival at the Earth detectors like SuperKamiokande [113], IceCube [114], etc can look for high energy muon neutrinos pointing back to the Sun.

Question 22. Capture of DM in the Sun, or elsewhere DM may be captured in the Sun, Earth, or other dense object. Although calculating the capture rate is challenging, as we have seen, it is straightforward to determine the evolution of number of DM particles with time. There are 3 competing effects, capture $\left(\Gamma_{c a p}\right)$, annihilation $\left(\Gamma_{a n n}\right)$, and evaporation $\left(\Gamma_{\text {evap }}\right)$. Without worrying about the details of the rate determine its scaling with the number of DM particles in the Sun, i.e. determine $p$ for each process with $\Gamma \sim N^{p}$. Then solve the evolution equation. Consider the limits where evaporation can be ignored, and where all processes are comparable.

\subsection{WIMPs at colliders}

The previous search strategies have relied upon measuring the ambient DM in one way or another. However, if DM is coupled to the SM, and light enough, then it may be possible to produce it at a sufficient rate to observe it at colliders like the LHC. There is a parity making the DM stable meaning it is produced in pairs. Furthermore, by definition, it has very feeble interactions with the SM so once produced it leaves the detector unmeasured. Thus, DM manifests itself as missing energy and momentum. At hadronic machines, where only transverse momentum, $\vec{p}_{T}$, can be reconstructed, DM looks like an imbalance among the $\vec{p}_{T}$ of the visible particles, often denoted $\mathbb{E}_{T}$.

Since DM has such weak interactions, in many models it is most easily made through the decay of some more strongly coupled state in the dark sector. For example, SUSY neutralino DM will appear as the final decay product of squarks or gluinos. Depending on the exact spectrum of the SUSY model this decay will result in various numbers of jets, leptons, electroweak bosons, and $\mathbb{E}_{T}$. There are numerous searches for strong production of SUSY particles that decay down to DM at both ATLAS [115] and CMS [116]. Bounds on strong production are now approaching several $\mathrm{TeV}$, if the neutralino is light. 
If an excess of events involving $\mathbb{E}_{T}$ is seen at the LHC we cannot be sure that the missing energy is really DM since the time it takes the particle to transit the detector is only a (rather weak) lower limit on its lifetime. Ultimately, if an excess is found, the dream will be to measure the properties and couplings of the new states with sufficient accuracy that we can determine if the particle associated with the $\mathbb{E}_{T}$ is truly the cosmological DM.

As the constraints on coloured superpartners become stronger it is tempting to imagine that perhaps they are beyond the reach of present machines but that the electroweakinos, which are produced at far lower rates, are still light. As we saw in Sections 4.3 and 5 the electroweakino sector provides a viable and interesting DM sector, where it is possible for the lightest neutralino to be a thermal relic. This remains true if the rest of the SUSY spectrum is decoupled [117, 56]. Restricting to the part of the four dimensional $\left(M_{1}, M_{2}, \mu\right.$, and $\left.\tan \beta\right)$ where the correct relic abundance is achieved one finds that, as discussed earlier, the splitting between the LSP and the NLSP is often small. The lack of coloured states and the small splitting among the lightest electroweakinos leads to a set of search strategies that we now briefly discuss.

Pair production of the lightest neutralinos leads to a very empty event. If the neutralino system is made in association with some hard initial state radiation (ISR), typically a gluon (jet) or photon then the $\chi$ 's will be boosted and the event will have a hard jet/photon and nothing else. Such mono-jet/photon events can be used to search for DM production in general models $[118,119]$ as well as in SUSY [120]. Production of the LSP in association with the lightest chargino can lead to the "disappearing track" signature. When the chargino-LSP mass splitting is small the chargino can be long lived and decays to the LSP and a soft pion after propagating a macroscopic distance. The pion is missed and what is observed is a charge track ending in the detector. For larger, but still $\Delta m \lesssim 50 \mathrm{GeV}$, splittings the decay from NLSP to LSP will be through off-shell electroweak gauge bosons and will result in soft leptons, another useful signature [120,121]. Finally, over the small splitting part of the parameter space even radiative decays, $\chi_{i} \rightarrow \chi_{j}+\gamma$, can be important [117]. A detailed discussion of the collider phenomenology of SUSY WIMPs, at present and future machines, is beyond the scope of these lectures, we refer the interested reader to the literature. However, a combination of direct detection, indirect detection and collider searches at a $100 \mathrm{TeV}$ machine will hopefully be able to uncover DM if it is a thermal relic neutralino [56].

Question 23. Relativistic limits, DM@colliders Consider fermionic DM $\chi$ coupled to SM fermions $f$. What are the production cross sections for DM at a collider through each of these interactions (a) $\bar{\chi} \chi \bar{f} f$, (b) $\bar{\chi} \gamma_{\mu} \gamma_{5} \chi \bar{f} \gamma^{\mu} \gamma_{5} f$, and (c) $\bar{\chi} \gamma_{\mu} \gamma_{5} \chi \bar{f} \gamma^{\mu} f$ ?

\section{Conclusions}

Unfinishedness avoids the stupidity of conclusions

-Pierre Senges

Although these lecture notes have only scratched the surface of what is (several) vast subject(s) it is my hope that they contain enough information (and motivation!) for you to set off on your own. Dark Matter is one of the biggest puzzles in present day particle physics and is under simultaneous assault from theorists and experimentalists. Supersymmetry is a beautiful idea that 
lays the groundwork for many of the scenarios of BSM physics. A breakthrough may occur in either area at any time and hopefully these lectures will help you prepare for it.

However, there are many topics that I didn't cover, because of lack of time, some of which will be covered elsewhere in this volume. Others will require independent study on your behalf. A large, and somewhat overwhelming, document that contains descriptions of the situation with regard to non-WIMP DM, as well as references to many classic papers is Ref. [122]. The "SUSY Primer" [27] is the best way into the supersymmetric world and will quickly fill in many of the details omitted in these lectures, as well as bring you close to the cutting edge. I encourage you to forge your own way through the DM and SUSY literature and hopefully these lectures can be your initial guide into this exciting realm.

\section{Acknowledgments}

I would like to thank Tracy Slatyer, Tilman Plehn and Tom DeGrand for organising TASI 2018 ("Theory in an Era of Data") and to thank Tracy and Tilman for their unwavering patience when preparing these notes. I would also like to thank Enrico Bertuzzo, Eduardo Pontón, Andrea Romanino, and Giovanni Villadoro for organizing the First Joint ICTP-Trieste/ICTP-SAIFR School on Particle Physics where parts of these lectures were presented. I thank the students at both schools for making the presentation of these lectures so enjoyable. This work was supported in part by the DoE under contract number DE-SC0007859 and Fermilab, operated by Fermi Research Alliance, LLC under contract number DE-AC02-07CH11359 with the United States Department of Energy.

\section{References}

[1] W. Hu, Lecture Notes on CMB Theory: From Nucleosynthesis to Recombination, 0802.3688.

[2] Planck collaboration, N. Aghanim et al., Planck 2018 results. VI. Cosmological parameters, 1807.06209.

[3] R. A. Alpher, H. Bethe and G. Gamow, The origin of chemical elements, Phys. Rev. 73 (Apr, 1948) 803-804.

[4] V. F. Mukhanov, Nucleosynthesis without a Computer, Int. J. Theor. Phys. 43 (2004) 669-693, [astro-ph/0303073].

[5] Ali-Haïmoud, Yacine and Kamionkowski, Marc, Cosmic microwave background limits on accreting primordial black holes, Phys. Rev. D95 (2017) 043534, [1612. 05644 ].

[6] Y. Hochberg, E. Kuflik, T. Volansky and J. G. Wacker, Mechanism for Thermal Relic Dark Matter of Strongly Interacting Massive Particles, Phys. Rev. Lett. 113 (2014) 171301, [1402 . 5143].

[7] E. Kuflik, M. Perelstein, N. R.-L. Lorier and Y.-D. Tsai, Elastically Decoupling Dark Matter, Phys. Rev. Lett. 116 (2016) 221302, [1512.04545].

[8] A. Hook, TASI Lectures on the Strong CP Problem and Axions, 1812.02669.

[9] E. W. Kolb and M. S. Turner, The Early Universe, Front. Phys. 69 (1990) 1-547.

[10] S. Dodelson, Modern Cosmology. Academic Press, Amsterdam, 2003.

[11] Particle Data Group collaboration, C. Patrignani et al., Review of Particle Physics, Chin. Phys. C40 (2016) 100001. 
[12] Y. Mambrini, "Histories of Dark Matter in the Universe."

http://www.ymambrini.com/My_World/Physics_files/Universe.pdf.

[13] F. Tanedo, "Defense against the Dark Arts."

http: //www.physics.uci.edu/ tanedo/files/notes/DMNotes.pdf.

[14] D. Hooper, Tasi 2008 Lectures on Dark Matter, 0901.4090.

[15] M. Lisanti, Lectures on Dark Matter Physics, in Proceedings, Theoretical Advanced Study Institute in Elementary Particle Physics: New Frontiers in Fields and Strings (TASI 2015): Boulder, CO, USA, June 1-26, 2015, pp. 399-446, 2017. 1603.03797. DOI.

[16] T. Plehn, Yet Another Introduction to Dark Matter, 1705.01987.

[17] J. M. Cline, TASI Lectures on Early Universe Cosmology: Inflation, Baryogenesis and Dark Matter, 1807.08749 .

[18] J. Bernstein, Kinetic Theory in the Expanding Universe. Cambridge University Press, Cambridge, U.K., 1988, 10.1017/CBO9780511564185.

[19] P. Gondolo and G. Gelmini, Cosmic abundances of stable particles: Improved analysis, Nucl. Phys. B360 (1991) 145-179.

[20] M. Cannoni, Relativistic $\left\langle\sigma v_{\text {rel }}\right\rangle$ in the calculation of relics abundances: a closer look, Phys. Rev. D89 (2014) 103533, [1311.4494].

[21] B. W. Lee and S. Weinberg, Cosmological lower bound on heavy-neutrino masses, Phys. Rev. Lett. 39 (Jul, 1977) 165-168.

[22] K. Griest and M. Kamionkowski, Unitarity Limits on the Mass and Radius of Dark Matter Particles, Phys. Rev. Lett. 64 (1990) 615.

[23] J. A. Bagger, “Weak-Scale Supersymmetry: Theory and Practice.” 1996.

[24] H. E. Haber, "Introductory Low-Energy Supersymmetry.” 1993.

[25] M. A. Luty, “2004 Tasi Lectures on Supersymmetry Breaking.” 2005.

[26] Y. Shirman, "Tasi 2008 Lectures: Introduction to Supersymmetry and Supersymmetry Breaking." 2009.

[27] S. P. Martin, A Supersymmetry primer, hep-ph/9709356.

[28] M. E. Peskin, “Supersymmetry in Elementary Particle Physics.” 2008.

[29] M. Perelstein, Introduction to Collider Physics, in TASI 2009, 2010.

[30] H. Baer and X. Tata, Weak Scale Supersymmetry: from Superfields to Scattering Events.

[31] J. Bagger and J. Wess, Supersymmetry and Supergravity.

[32] https://en.wikipedia.org/wiki/Esperanto.

[33] S. R. Coleman and J. Mandula, All Possible Symmetries of the s Matrix, Phys. Rev. 159 (1967) 1251-1256.

[34] R. Haag, J. T. Lopuszanski and M. Sohnius, All Possible Generators of Supersymmetries of the s Matrix, Nucl. Phys. B88 (1975) 257.

[35] H. K. Dreiner, H. E. Haber and S. P. Martin, Two-component spinor techniques and Feynman rules for quantum field theory and supersymmetry, 0812.1594. 
[36] J. Polonyi, "Budapest Preprint KFKI-93.” 1977.

[37] L. O’Raifeartaigh, Spontaneous Symmetry Breaking for Chiral Scalar Superfields, Nucl. Phys. B96 (1975) 331.

[38] T. Appelquist, H.-C. Cheng and B. A. Dobrescu, Bounds on Universal Extra Dimensions, Phys. Rev. D64 (2001) 035002, [hep-ph/ 0012100$].$

[39] H.-C. Cheng and I. Low, TeV Symmetry and the Little Hierarchy Problem, JHEP 09 (2003) 051, [hep-ph/0308199].

[40] L. E. Ibanez and G. G. Ross, Discrete Gauge Symmetries and the Origin of Baryon and Lepton Number Conservation in Supersymmetric Versions of the Standard Model, Nucl. Phys. $\mathbf{3 3 6 8}$ (1992) $3-37$.

[41] H. K. Dreiner, C. Luhn and M. Thormeier, What is the Discrete Gauge Symmetry of the Mssm?, Phys. Rev. D73 (2006) 075007, [hep-ph / 0512163$].$

[42] H. Murayama and A. Pierce, Not Even Decoupling Can Save Minimal Supersymmetric SU(5), Phys. Rev. D65 (2002) 055009, [hep-ph/ 0108104 ].

[43] S. Dimopoulos and D. W. Sutter, The Supersymmetric Flavor Problem, Nucl. Phys. B452 (1995) 496-512, [hep-ph/9504415].

[44] D. Shih, Dynamical supersymmetry breaking, in TASI 2009, 2010.

[45] P. Meade, Gauge mediation of supersymmetry breaking, in TASI 2009, 2010.

[46] G. F. Giudice and A. Masiero, A Natural Solution to the Mu Problem in Supergravity Theories, Phys. Lett. B206 (1988) 480-484.

[47] L. Girardello and M. T. Grisaru, Soft Breaking of Supersymmetry, Nucl. Phys. B194 (1982) 65.

[48] P. J. Fox, A. E. Nelson and N. Weiner, Dirac Gaugino Masses and Supersoft Supersymmetry Breaking, JHEP 08 (2002) 035, [hep-ph/ 0206096 ].

[49] J. Polchinski and L. Susskind, Breaking of Supersymmetry at Intermediate-Energy, Phys. Rev. D26 (1982) 3661.

[50] D. R. T. Jones, L. Mezincescu and Y. P. Yao, Soft Breaking of Two Loop Finite $\mathscr{N}=1$ Supersymmetric Gauge Theories, Phys. Lett. B148 (1984) 317-322.

[51] L. J. Hall and L. Randall, Weak Scale Effective Supersymmetry, Phys. Rev. Lett. 65 (1990) 2939-2942.

[52] M. Dine and D. MacIntire, Supersymmetry, Naturalness, and Dynamical Supersymmetry Breaking, Phys. Rev. D46 (1992) 2594-2601, [hep-ph/9205227].

[53] I. Jack and D. R. T. Jones, Non-Standard Soft Supersymmetry Breaking, Phys. Lett. B457 (1999) 101-108, [hep-ph/9903365].

[54] Z. Chacko, P. J. Fox and H. Murayama, Localized Supersoft Supersymmetry Breaking, Nucl. Phys. B706 (2005) 53-70, [hep-ph / 0406142$].$

[55] L. J. Hall, T. Moroi and H. Murayama, Sneutrino cold dark matter with lepton number violation, Phys. Lett. B424 (1998) 305-312, [hep-ph/9712515].

[56] J. Bramante, N. Desai, P. Fox, A. Martin, B. Ostdiek and T. Plehn, Towards the Final Word on Neutralino Dark Matter, Phys. Rev. D93 (2016) 063525, [1510.03460]. 
[57] M. Cirelli, N. Fornengo and A. Strumia, Minimal dark matter, Nucl. Phys. B753 (2006) 178-194, [hep-ph/0512090].

[58] J. Bramante, P. J. Fox, G. D. Kribs and A. Martin, Inelastic frontier: Discovering dark matter at high recoil energy, Phys. Rev. D94 (2016) 115026, [1608.02662].

[59] D. Tucker-Smith and N. Weiner, Inelastic dark matter, Phys. Rev. D64 (2001) 043502, [hep-ph/0101138].

[60] J. Hisano, K. Ishiwata, N. Nagata and T. Takesako, Direct Detection of Electroweak-Interacting Dark Matter, JHEP 07 (2011) 005, [1104.0228].

[61] R. J. Hill and M. P. Solon, WIMP-nucleon scattering with heavy WIMP effective theory, Phys. Rev. Lett. 112 (2014) 211602, [1309.4092].

[62] J. L. Feng, A. Rajaraman and F. Takayama, Superweakly interacting massive particles, Phys. Rev. Lett. 91 (2003) 011302, [hep-ph/0302215].

[63] K. Jedamzik, Big bang nucleosynthesis constraints on hadronically and electromagnetically decaying relic neutral particles, Phys. Rev. D74 (2006) 103509, [hep-ph/ 0604251$].$

[64] A. E. Nelson, N. Rius, V. Sanz and M. Unsal, The Minimal Supersymmetric Model without a Mu Term, JHEP 08 (2002) 039, [hep-ph/0206102].

[65] G. Jungman, M. Kamionkowski and K. Griest, Supersymmetric dark matter, Phys. Rept. 267 (1996) 195-373, [hep-ph/9506380].

[66] K. Griest and D. Seckel, Three exceptions in the calculation of relic abundances, Phys. Rev. D43 (1991) 3191-3203.

[67] J. Edsjo and P. Gondolo, Neutralino relic density including coannihilations, Phys. Rev. D56 (1997) 1879-1894, [hep-ph/9704361].

[68] P. Gondolo et al., Darksusy: Computing Supersymmetric Dark Matter Properties Numerically, JCAP 0407 (2004) 008, [astro-ph/ 040620 4].

[69] G. Belanger, F. Boudjema, A. Pukhov and A. Semenov, Micromegas 2.0.7: a Program to Calculate the Relic Density of Dark Matter in a Generic Model, Comput. Phys. Commun. 177 (2007) 894-895.

[70] M. W. Goodman and E. Witten, Detectability of Certain Dark Matter Candidates, Phys. Rev. D31 (1985) 3059.

[71] CDMS Collaboration collaboration, R. Agnese et al., Dark Matter Search Results Using the Silicon Detectors of CDMS II, Phys.Rev.Lett. (2013), [1304.4279].

[72] SuPERCDMS collaboration, R. Agnese et al., Results from the Super Cryogenic Dark Matter Search Experiment at Soudan, Phys. Rev. Lett. 120 (2018) 061802, [1708. 08869 ].

[73] CoGeNT collaboration, C. E. Aalseth et al., Results from a Search for Light-Mass Dark Matter with a P-type Point Contact Germanium Detector, Phys. Rev. Lett. 106 (2011) 131301, [1 002 . 4703 ].

[74] COUPP collaboration, E. Behnke et al., First Dark Matter Search Results from a 4-kg CF $I_{3}$ Bubble Chamber Operated in a Deep Underground Site, Phys. Rev. D86 (2012) 052001, [1204. 3094 ].

[75] CRESST collaboration, G. Angloher et al., Results on light dark matter particles with a low-threshold CRESST-II detector, Eur. Phys. J. C76 (2016) 25, [1509.01515].

[76] DAMA collaboration, R. Bernabei et al., First results from DAMA/LIBRA and the combined results with DAMA/NaI, Eur. Phys. J. C56 (2008) 333-355, [0804.2741]. 
[77] KIMS collaboration, K. W. Kim et al., Limits on Interactions between Weakly Interacting Massive Particles and Nucleons Obtained with NaI(Tl) crystal Detectors, 1806.06499.

[78] XENON100 collaboration, E. Aprile et al., XENON100 Dark Matter Results from a Combination of 477 Live Days, Phys. Rev. D94 (2016) 122001, [1609.06154].

[79] XENON collaboration, E. Aprile et al., Dark Matter Search Results from a One Ton-Year Exposure of XENON1T, Phys. Rev. Lett. 121 (2018) 111302, [1805.12562].

[80] J. D. Lewin and P. F. Smith, Review of mathematics, numerical factors, and corrections for dark matter experiments based on elastic nuclear recoil, Astropart. Phys. 6 (1996) 87-112.

[81] J. I. Read, The Local Dark Matter Density, J. Phys. G41 (2014) 063101, [1404.1938].

[82] M. Schumann, Direct Detection of WIMP Dark Matter: Concepts and Status, 1903.03026.

[83] J. Bovy and S. Tremaine, On the local dark matter density, Astrophys. J. 756 (2012) 89, [1205.4033].

[84] R. Catena and P. Ullio, A novel determination of the local dark matter density, JCAP 1008 (2010) 004, [0907.0018].

[85] M. Kuhlen, M. Vogelsberger and R. Angulo, Numerical Simulations of the Dark Universe: State of the Art and the Next Decade, Phys. Dark Univ. 1 (2012) 50-93, [1209. 5745].

[86] M. Kuhlen, N. Weiner, J. Diemand, P. Madau, B. Moore, D. Potter et al., Dark Matter Direct Detection with Non-Maxwellian Velocity Structure, JCAP 1002 (2010) 030, [0 912 . 2358].

[87] P. J. Fox, G. D. Kribs and T. M. P. Tait, Interpreting Dark Matter Direct Detection Independently of the Local Velocity and Density Distribution, Phys. Rev. D83 (2011) 034007, [1011.1910].

[88] P. J. Fox, J. Liu and N. Weiner, Integrating Out Astrophysical Uncertainties, Phys. Rev. D83 (2011) 103514, [1011.1915].

[89] A. K. Drukier, K. Freese and D. N. Spergel, Detecting Cold Dark Matter Candidates, Phys. Rev. D33 (1986) 3495-3508.

[90] N. W. Evans, C. A. O'Hare and C. McCabe, Refinement of the standard halo model for dark matter searches in light of the Gaia Sausage, Phys. Rev. D99 (2019) 023012, [1810 . 11468].

[91] C. McCabe, The Earth's velocity for direct detection experiments, JCAP 1402 (2014) 027, [1312.1355].

[92] D. Tucker-Smith and N. Weiner, The Status of inelastic dark matter, Phys. Rev. D72 (2005) 063509, [hep-ph/0402065].

[93] S. Chang, G. D. Kribs, D. Tucker-Smith and N. Weiner, Inelastic Dark Matter in Light of DAMA/LIBRA, Phys. Rev. D79 (2009) 043513, [0 807 .2250].

[94] P. Junnarkar and A. Walker-Loud, Scalar strange content of the nucleon from lattice QCD, Phys. Rev. D87 (2013) 114510, [1301.1114].

[95] Particle Data Group collaboration, M. Tanabashi, K. Hagiwara, K. Hikasa, K. Nakamura, Y. Sumino, F. Takahashi et al., Review of particle physics, Phys. Rev. D 98 (Aug, 2018) 030001.

[96] C. Cheung, L. J. Hall, D. Pinner and J. T. Ruderman, Prospects and Blind Spots for Neutralino Dark Matter, JHEP 05 (2013) 100, [1211.4873].

[97] P. Huang and C. E. M. Wagner, Blind Spots for neutralino Dark Matter in the MSSM with an intermediate $m_{A}$, Phys. Rev. D90 (2014) 015018, [1404.0392]. 
[98] T. Han, F. Kling, S. Su and Y. Wu, Unblinding the dark matter blind spots, JHEP 02 (2017) 057, [1612.02387].

[99] M. Cirelli, G. Corcella, A. Hektor, G. Hutsi, M. Kadastik, P. Panci et al., PPPC 4 DM ID: A Poor Particle Physicist Cookbook for Dark Matter Indirect Detection, JCAP 1103 (2011) 051, [1012.4515].

[100] D. Hooper, TASI Lectures on Indirect Searches For Dark Matter, 1812.02029.

[101] J. F. Navarro, C. S. Frenk and S. D. M. White, The Structure of cold dark matter halos, Astrophys. J. 462 (1996) 563-575, [astro-ph/9508025].

[102] J. Einasto, Kinematics and dynamics of stellar systems, , Trudy Inst. Astrofiz. Alma-Ata 5 (1965) 87.

[103] A. W. Graham, D. Merritt, B. Moore, J. Diemand and B. Terzic, Empirical models for Dark Matter Halos. I. Nonparametric Construction of Density Profiles and Comparison with Parametric Models, Astron. J. 132 (2006) 2685-2700, [astro-ph/ 0509417$].$

[104] A. Burkert, The Structure of dark matter halos in dwarf galaxies, IAU Symp. 171 (1996) 175, [astro-ph/9504041].

[105] T. Sjostrand, S. Mrenna and P. Z. Skands, A Brief Introduction to PYTHIA 8.1, Comput. Phys. Commun. 178 (2008) 852-867, [0710.3820].

[106] L. Goodenough and D. Hooper, Possible Evidence For Dark Matter Annihilation In The Inner Milky Way From The Fermi Gamma Ray Space Telescope, 0910.2998.

[107] D. Hooper and L. Goodenough, Dark Matter Annihilation in The Galactic Center As Seen by the Fermi Gamma Ray Space Telescope, Phys. Lett. B697 (2011) 412-428, [1010 . 2752].

[108] A. Gould, WIMP Distribution in and Evaporation From the Sun, Astrophys. J. 321 (1987) 560.

[109] A. Gould, Resonant Enhancements in WIMP Capture by the Earth, Astrophys. J. 321 (1987) 571.

[110] P. Baratella, M. Cirelli, A. Hektor, J. Pata, M. Piibeleht and A. Strumia, PPPC 4 DMv: a Poor Particle Physicist Cookbook for Neutrinos from Dark Matter annihilations in the Sun, JCAP 1403 (2014) 053, [1312.6408].

[111] W. H. Press and D. N. Spergel, Capture by the sun of a galactic population of weakly interacting massive particles, Astrophys. J. 296 (1985) 679-684.

[112] L. M. Krauss, K. Freese, W. Press and D. Spergel, Cold dark matter candidates and the solar neutrino problem, Astrophys. J. 299 (1985) 1001.

[113] SuPER-KAMIOKANDE collaboration, K. Choi et al., Search for neutrinos from annihilation of captured low-mass dark matter particles in the Sun by Super-Kamiokande, Phys. Rev. Lett. 114 (2015) 141301, [1503.04858].

[114] ICECUBE collaboration, M. G. Aartsen et al., Search for annihilating dark matter in the Sun with 3 years of IceCube data, Eur. Phys. J. C77 (2017) 146, [1612 . 05949].

[115] ATLAS Collaboration, "SUSY searches." https://twiki.cern.ch/twiki/bin/view/ AtlasPublic/SupersymmetryPublicResults.

[116] CMS Collaboration, "SUSY searches." http://cms-results.web.cern.ch/ cms-results/public-results/publications/SUS/index.html.

[117] J. Bramante, P. J. Fox, A. Martin, B. Ostdiek, T. Plehn, T. Schell et al., Relic Neutralino Surface at a 100ăTeV Collider, Phys. Rev. D91 (2015) 054015, [1412.4789]. 
[118] M. Beltran, D. Hooper, E. W. Kolb, Z. A. C. Krusberg and T. M. P. Tait, Maverick dark matter at colliders, JHEP 09 (2010) 037, [1002.4137].

[119] P. J. Fox, R. Harnik, J. Kopp and Y. Tsai, Missing Energy Signatures of Dark Matter at the LHC, Phys. Rev. D85 (2012) 056011, [1109.4398].

[120] M. Low and L.-T. Wang, Neutralino dark matter at 14 TeV and $100 \mathrm{TeV}, \mathrm{JHEP} 08$ (2014) 161, [1404.0682].

[121] M. Cirelli, F. Sala and M. Taoso, Wino-like Minimal Dark Matter and future colliders, JHEP 10 (2014) 033, [1407.7058].

[122] M. Battaglieri et al., US Cosmic Visions: New Ideas in Dark Matter 2017: Community Report, 1707.04591. 